Michael Struwe

\title{
Positive solutions of critical semilinear elliptic equations on non-contractible planar domains
}

Received July 19, 1999 / final version received April 25, 2000

Published online July 27, 2000 - (c) Springer-Verlag \& EMS 2000

Abstract. For semilinear elliptic equations of critical exponential growth we establish the existence of positive solutions to the Dirichlet problem on suitable non-contractible domains.

1.

For a smooth, bounded domain $\Omega \subset \mathbb{R}^{2}$ consider the semilinear equation

$$
-\Delta u=f(u) \text { in } \Omega, u=0 \text { on } \partial \Omega,
$$

where $f: \mathbb{R} \rightarrow \mathbb{R}$ is smooth and has critical exponential growth. For example, we may consider

$$
f(u)=u e^{4 \pi u^{2}}
$$

with primitive

$$
F(u)=\int_{0}^{u} f(u) d v=\frac{1}{8 \pi}\left(e^{4 \pi u^{2}}-1\right)
$$

In this case, as shown in [1], p. 394, problem (1) always admits a positive solution whenever the diameter of $\Omega$ is sufficiently small.

On the other hand, in [4] it is shown that there exists $R_{0}>0$ such that for the function

$$
f(u)=u e^{4 \pi u^{2}-u}
$$

the Dirichlet problem (1) does not admit a solution $u>0$ on any ball $B_{R}(0)$ with $R<R_{0}$; see also [3] for further results in this regard.

Thus, the existence of positive solutions to problem (1) depends on the nonlinearity in a very subtle way, as is characteristic of critical variational

M. Struwe: Mathematik, ETH-Zentrum, 8092 Zürich, Switzerland, e-mail: struwe@math.ethz.ch 
problems. In higher dimensions $n \geq 3$, the analogous behavior is observed in the equation

$$
-\Delta u=|u|^{2^{*}-2} u+\lambda u \text { on } \Omega, u=0 \text { on } \partial \Omega,
$$

on a domain $\Omega \subset \subset \mathbb{R}^{n}$, where $2^{*}=\frac{2 n}{n-2}$ is the limiting exponent for the Sobolev embedding $H_{0}^{1}(\Omega) \hookrightarrow L^{p}(\Omega), 1 \leq p \leq 2^{*}$, as we vary the parameter $\lambda \geq 0$; see [23], Chap. 3, for a survey of results.

For a planar domain $\Omega \subset \subset \mathbb{R}^{2}$, the analogue of the critical Sobolev embedding $H_{0}^{1}(\Omega) \hookrightarrow L^{2^{*}}(\Omega)$ in dimensions $n \geq 3$ is the Orlicz space embedding

$$
H_{0}^{1}(\Omega) \ni u \mapsto e^{u^{2}} \in L^{p}(\Omega)
$$

for all $p<\infty$. In particular, we have the Moser-Trudinger inequality

$$
\sup _{u \in H_{0}^{1}(\Omega) ;\|u\|_{H_{0}^{1}(\Omega)} \leq 1} \int_{\Omega} e^{4 \pi u^{2}} d x<\infty,
$$

where $\|u\|_{H_{0}^{1}(\Omega)}^{2}=\int_{\Omega}|\nabla u|^{2} d x$; see [18], [24].

In view of the Moser-Trudinger inequality the functional

$$
E(u)=\frac{1}{2} \int_{\Omega}|\nabla u|^{2} d x-\int_{\Omega} F(u) d x
$$

associated with (1) then is well-defined and smooth on $H_{0}^{1}(\Omega)$ and critical points $u \in H_{0}^{1}(\Omega)$ of $E$ are classical $\left(C^{\infty}\right.$ ) solutions of (1). However, the functional $E$ fails to satisfy the Palais-Smale condition.

In the present paper we show that - similar to results of Coron [11], Bahri-Coron [6] for equation (4) in dimensions $n \geq 3$ - positive solutions to the boundary value problem (1), (3) always exist on suitable non-contractible domains, as was suggested by Adimurthi-Prashanth [2]. In fact, the result is true for a large class of nonlinearities $f$ of critical exponential growth.

More precisely, we shall study nonlinearities of the form

$$
f(u)=u e^{\varphi(u)}
$$

with primitive $F$, defined in terms of a smooth function $\varphi: \mathbb{R} \rightarrow \mathbb{R}$ having the properties

$$
\begin{gathered}
\varphi(0)=0, \varphi(s) \leq 1 \text { for } s \leq 0, \varphi(s) \leq 4 \pi s^{2} \text { for } s \geq 0, \\
-1 \leq \varphi^{\prime}(s) s \leq 8 \pi s^{2}, \lim _{s \rightarrow \infty} \varphi^{\prime}(s) / s=8 \pi, \varphi^{\prime \prime}(s) \leq 8 \pi,
\end{gathered}
$$

and such that $\varphi$ and the function $s \mapsto s^{2} e^{\varphi(s)}$ are convex for $s \geq 0$. 
Observe that exponential growth implies that, in particular, with error $o(1) \rightarrow 0$ as $s \rightarrow \infty$,

$$
F(s) \leq o(1) s f(s) .
$$

Our main result can now be stated as follows.

Theorem 1.1. Let $f$ be given by (8), assuming (9). Then for suitable numbers $R_{0} \geq R_{1}>R_{2}>0$ problem (1) admits a positive solution on any domain $\Omega \subset B_{R_{0}}(0)$ containing the annulus $B_{R_{1}} \backslash B_{R_{2}}(0)$ and such that $0 \notin \bar{\Omega}$.

We expect a similar result to hold for any nonlinearity $f$ of critical exponential growth as defined in [1], Definition 2.1. Moreover, analogous to the Bahri-Coron result Theorem 1.1 should hold true for any non-contractible domain $\Omega \subset \subset \mathbb{R}^{2}$.

We conclude this introduction with some comments on the proof of Theorem 1.1, which is quite delicate. The approach of Coron in the higherdimensional case relies on the precise analysis of Palais-Smale sequences for the associated variational problem, carried out in [20], and, in particular, the precise characterization of the energy levels where the Palais-Smale condition fails to hold. For a domain $\Omega \subset \subset \mathbb{R}^{n}$ containing a non-contractible spherical shell $B_{R_{1}} \backslash B_{R_{2}}(0)$ with suitable radii $R_{1}>R_{2}$ a mountain-pass type construction then yields a Palais-Smale sequence with energy bounded away from these exceptional energy levels which therefore accumulates at a critical point.

This strategy cannot be carried over to two-dimensional critical problems directly, because, as shown in [2], [5], in two space dimensions the behavior of Palais-Smale sequences is more complicated than in the higherdimensional case. However, in [5] we demonstrated that the situation improves if we consider solutions $u_{k}$ to equations of type (1) with nonlinearities $f_{k}(s)=s e^{\varphi_{k}(s)}$ defined in terms of functions $\varphi_{k}$ satisfying condition (9); in fact, at energy levels allowing at most single-point blow-up we were able to analyze the concentration behavior of $\left(u_{k}\right)$ completely. Indeed, at a concentration point after suitable (nonlinear) rescaling there emerges a unique blow-up profile that has the same geometric interpretation as the blow-up profile in the case of the Moser-Trudinger embedding (5), analyzed in [21].

However, this result by itself is not sufficient to prove Theorem 1.1, as the existence of suitable approximate solutions could only be asserted if Theorem 1.1 already was known to hold true for a sufficiently large class of functions $\varphi$.

Also the natural strategy of approximating equation (1) by sub-critical semilinear equations of type (1) with nonlinearities $f(s)=s e^{\varphi(s)}$ where $\varphi$ has sub-quadratic growth does not seem to work because it appears to be 
impossible to control the energy levels where concentration may occur in this case.

Instead, in the present paper we adopt an approximation strategy of Sacks-Uhlenbeck [19] and consider, for $\alpha>1$, the problem

$$
-\operatorname{div}\left[\left(1+|\nabla u|^{2}\right)^{\alpha-1} \nabla u\right]=f(u) \text { in } \Omega, u=0 \text { on } \partial \Omega,
$$

with associated energy

$$
E_{\alpha}(u)=\frac{1}{2 \alpha} \int_{\Omega}\left[\left(1+|\nabla u|^{2}\right)^{\alpha}-1\right] d x-\int_{\Omega} F(u) d x,
$$

whose critical points $u_{\alpha} \in W_{0}^{1,2 \alpha}(\Omega)$ again are smooth solutions of (11).

It is easily verified that (11) admits a positive solution for sufficiently small $\alpha>1$ on any sufficiently small domain $\Omega$; see Lemma 3.6 below. However, these solutions may degenerate as $\alpha \searrow 1$.

On the other hand, if we assume - as we may, for otherwise Theorem 1.1 trivially is true - that our original problem (1) does not admit a solution $u>0$ with $E(u) \leq \frac{1}{2}$, then for a sufficiently small, non-contractible domain Coron's method may be applied to show that equation (11) for sufficiently small $\alpha>1$ also admits solutions $u_{\alpha}$ of saddle type, whose energies $E_{\alpha}\left(u_{\alpha}\right)=\beta_{\alpha}$ monotonically decrease to a limit $\beta>\frac{1}{2}$ as $\alpha \rightarrow 1$. Restricting the shape of $\Omega$ slightly more, we also obtain the upper bound $\beta<1$. Monotone dependence on the parameter $\alpha>1$ may be exploited in a way similar to [21], [22], where this idea was first conceived, to derive additional a-priori bounds on $u_{\alpha}$ for a sequence $\alpha \rightarrow 1$.

We summarize these results in our second theorem.

Theorem 1.2. For numbers $R_{0} \geq R_{1}>R_{2}>0$ let $\Omega \subset B_{R_{0}}(0) \subset \mathbb{R}^{2}$ be a smoothly bounded domain containing the annulus $B_{r_{1}} \backslash B_{R_{2}}(0)$ and such that $0 \notin \bar{\Omega}$, and suppose assumption (21) below is satisfied.

Then, if $R_{0}$ is sufficiently small and if, given $R_{0}$ and $R_{1} \leq R_{0}$, the number $R_{2}$ is sufficiently small, there are numbers $\left.\alpha_{0}>1, \beta \in\right] \frac{1}{2}, 1[$ such that for any $\alpha \in] 1, \alpha_{0}$ [ equation (11) admits a positive solution $u_{\alpha} \in W_{0}^{1,2 \alpha}(\Omega)$, with energy $E_{\alpha}\left(u_{\alpha}\right)=\beta_{\alpha} \rightarrow \beta$ monotonically as $\alpha \rightarrow 1$.

In addition, we can achieve that

$$
\liminf _{\alpha \rightarrow 1}\left((\alpha-1) \log \left(\frac{1}{\alpha-1}\right) \frac{\partial E_{\alpha}}{\partial \alpha}\left(u_{\alpha}\right)\right)=0 .
$$

Using results from the theory of quasilinear elliptic equations as presented in [8] we then succeed in carrying over the blow-up analysis from [5] to the sequence $\left(u_{\alpha}\right)$ and to identify the energy level where blow-up may occur. The "entropy" bound (12) is crucial in this last step. 
More precisely, we have the following result.

Theorem 1.3. Suppose assumption (21) holds true. For $1<\alpha<\alpha_{0}$ let $u_{\alpha} \in W_{0}^{1,2 \alpha}(\Omega)$ be positive solutions to equation (11) with energy $E_{\alpha}\left(u_{\alpha}\right)=$ $\beta_{\alpha} \rightarrow \beta<1$ as $\alpha \rightarrow 1$ and satisfying (12).

Then, either i) as $\alpha \rightarrow 1$ a sequence $\left(u_{\alpha}\right)$ converges strongly in $H_{0}^{1}(\Omega)$ to a solution $u$ of (1) with energy $E(u)=\beta$, or ii) $\beta=\frac{1}{2}$ and the following happens.

There exists a sequence $\alpha \rightarrow 1$ with corresponding points $x_{\alpha} \rightarrow x_{0} \in \bar{\Omega}$ and radii $r_{\alpha} \rightarrow 0$ such that, as $\alpha \rightarrow 1$,

$$
\left|\nabla u_{\alpha}\right|^{2 \alpha} d x \rightarrow \delta_{x_{0}},\left|\nabla u_{\alpha}\right|^{2} d x \rightarrow \delta_{x_{0}}, u_{\alpha} f\left(u_{\alpha}\right) d x \rightarrow \delta_{x_{0}}
$$

weakly in the sense of measures, and

$$
\begin{aligned}
\eta_{\alpha}(x): & =\varphi\left(u_{\alpha}\left(x_{\alpha}+r_{\alpha} x\right)\right)+2 \log \left(r_{\alpha} u_{\alpha}\left(x_{\alpha}\right)\right)+\log (8 \pi) \rightarrow \eta(x) \\
& =\log \left(\frac{1}{\left(1+|x|^{2} / 8\right)^{2}}\right)
\end{aligned}
$$

locally $C^{1}$-uniformly on $\mathbb{R}^{2}$, where $\eta$ is a solution of Liouville's equation

$$
-\Delta \eta=e^{\eta} \text { on } \mathbb{R}^{2} .
$$

Theorem 1.1 follows from Theorems 1.2 and 1.3.

\section{Preliminaries}

Besides the Moser-Trudinger inequality (6) the following variant of this estimate, due to Chang-Yang [9], will play a fundamental role in our argument. Let $f . .$. denote mean value.

Theorem 2.1. There exists a constant $C>0$ such that for any $R>0$, any $w \in H^{1}\left(B_{R}(0)\right)$ satisfying $\int_{B_{R}(0)} w d x=0, \int_{B_{R}(0)}|\nabla w|^{2} d x \leq 1$ there holds

$$
f_{B_{R}(0)} e^{2 \pi w^{2}} d x \leq C
$$

Moreover, we will need the analogue of [7], Theorem 1, for equation (11). Similar qualitative results, but with bounds possibly depending on the $L^{\infty}$-norm of the coefficients $a(x)$, were given by Chanillo-Li, Boccardo, Fiorenza-Sbordone, and others; see Fiorenza-Sbordone [14] for references. 
Theorem 2.2. Let $v$ be a solution to the equation

$$
-\operatorname{div}(a(x) \nabla v)=f \text { in } \Omega, v=0 \text { on } \partial \Omega,
$$

where $1 \leq a \in C^{\infty}(\bar{\Omega})$ and $f \in L^{1}(\Omega)$. Then for every $p<4 \pi\|f\|_{L^{1}(\Omega)}^{-1}$ there holds $e^{p v} \in L^{1}(\Omega)$ and

$$
f_{\Omega} e^{p v} d x \leq C(p)
$$

Moreover, $v \in W_{0}^{1, q}(\Omega)$ for any $q<2$ and

$$
\|v\|_{W_{0}^{1, q}(\Omega)} \leq C\left(q,\|f\|_{L^{1}(\Omega)}\right) .
$$

For the proof we first observe that (14) is a consequence of an estimate for the size of the level-sets of $v$. Let $\mu$ denote Lebesgue measure on $\mathbb{R}^{2}$.

Lemma 2.3. Suppose that for every $t>0$ there holds

$$
\mu(\{x \in \Omega ; v(x) \geq t\}) \leq A \exp (-B t) \mu(\Omega)
$$

with uniform constants $A, B \in \mathbb{R}$. Then (14) holds for any $p<B$ with $C \leq e^{p}+2 A q^{\frac{B-p}{2}} /((B-p) \log q)$, where $q=\frac{p+B}{2 p}$.

Proof. Let $q=\frac{p+B}{2 p}>1$. Then

$$
\begin{aligned}
\int_{\{x ; v(x) \geq 1\}} e^{p v} d x & \leq \sum_{k \geq 0} e^{p q^{k+1}} \mu\left(\left\{x ; q^{k} \leq v(x) \leq q^{k+1}\right\}\right) \\
& \leq \sum_{k \geq 0} e^{p q^{k+1}} \mu\left\{x ; q^{k} \leq v(x)\right\} \leq A \sum_{k \geq 0} e^{(p q-B) q^{k}} \mu(\Omega) .
\end{aligned}
$$

Note that $B-p q=\frac{B-p}{2}>0$. Thus, and since $q^{k} \leq \exp \left(q^{k}\right)$ for all $k \geq 0$, we may estimate

$$
\sum_{k \geq 0} e^{(p q-B) q^{k}} \leq \sum_{k \geq 0} q^{(p q-B) k}=\frac{1}{1-q^{p q-B}} \leq \frac{2 q^{\frac{B-p}{2}}}{(B-p) \log q} .
$$

The lemma follows.

Proof of Theorem 2.2. By density of $L^{2}(\Omega)$ in the space $L^{1}(\Omega)$ and continuous dependence of the solution $v$ to $(13)$ in $W_{0}^{1, q}(\Omega)$ on $f$ in $L^{1}(\Omega)$ for any $q<2$, we may assume that $f \in L^{2}(\Omega)$ and $v \in H_{0}^{1}(\Omega)$.

Moreover, by the weak maximum principle for (13) it suffices to prove (14) in the case $f \geq 0$; hence also $v \geq 0$. 
Fix $t>0$. Multiplying (13) by the truncated function $\min \{v, t\} \in H_{0}^{1}(\Omega)$ and integrating by parts, we obtain

$$
\int_{\{x ; v(x) \leq t\}}|\nabla v|^{2} d x \leq \int_{\Omega} f \min \{v, t\} d x \leq t\|f\|_{L^{1}(\Omega)} .
$$

The function $w=t^{-1} \min \{v, t\} \in H_{0}^{1}(\Omega)$ then satisfies $0 \leq w \leq 1$ in $\Omega, w=1$ on $\Omega_{t}=\{x ; v(x) \geq t\}$ and

$$
\|w\|_{H_{0}^{1}(\Omega)}^{2}=t^{-2} \int_{\{x ; v(x) \leq t\}}|\nabla v|^{2} d x \leq t^{-1}\|f\|_{L^{1}(\Omega)} .
$$

Let $D=B_{\rho}(0)$ be a ball of the same area as $\Omega_{t}$. Also choose $R>0$ such that $\mu\left(B_{R}(0)\right)=\mu(\Omega)$. Since the Dirichlet energy does not increase under radially symmetric rearrangement, we can estimate the capacity of $D$ relative to $B_{R}(0)$ as

$$
\begin{aligned}
\operatorname{cap}\left(D, B_{R}(0)\right) & =\inf \left\{\int_{B_{R}(0)}|\nabla \psi|^{2} d x ; \psi \in H_{0}^{1}\left(B_{R}(0)\right), \psi \equiv 1 \text { on } D\right\} \\
& \leq \inf \left\{\int_{\Omega}|\nabla \psi|^{2} d x ; \psi \in H_{0}^{1}(\Omega), \psi \equiv 1 \text { on } \Omega_{t}\right\} \\
& \leq \int_{\Omega}|\nabla w|^{2} d x \leq t^{-1}\|f\|_{L^{1}(\Omega)} .
\end{aligned}
$$

On the other hand, cap $\left(D, B_{R}(0)\right)$ is achieved by letting $\psi=\log \left(\frac{R}{r}\right) / \log \left(\frac{R}{\rho}\right)$, which gives

$$
\frac{2 \pi}{\log \left(\frac{R}{\rho}\right)}=\|\psi\|_{H_{0}^{1}\left(B_{R}(0)\right)}^{2}=\operatorname{cap}\left(D, B_{R}(0)\right) \leq t^{-1}\|f\|_{L^{1}(\Omega)} .
$$

Solving for $\mu(D)=\pi \rho^{2}$, we obtain

$$
\begin{aligned}
\mu\left(\Omega_{t}\right) & =\mu(D)=\pi \rho^{2} \leq \pi R^{2} \exp \left(-4 \pi t\|f\|_{L^{1}(\Omega)}^{-1}\right) \\
& =\mu(\Omega) \exp \left(-4 \pi t\|f\|_{L^{1}(\Omega)}^{-1}\right),
\end{aligned}
$$

that is, (16) with $A=1, B=4 \pi\|f\|_{L^{1}(\Omega)}^{-1}$. The first assertion of Theorem 2.2 now follows from Lemma 2.3.

To obtain a bound for $v$ in $W_{0}^{1, q}(\Omega)$ for any $q<2$, observe that (17) implies

$$
\int_{\left\{x ; 2^{k} \leq v(x) \leq 2^{k+1}\right\}} \frac{|\nabla v|^{2}}{v} d x \leq 2\|f\|_{L^{1}(\Omega)} .
$$

Dividing by $2^{k}$ and summing over $k \geq 0$, together with (17) for $t=1$ we obtain

$$
\int_{\Omega} \frac{|\nabla v|^{2}}{1+v^{2}} d x \leq 3\|f\|_{L^{1}(\Omega)}
$$


Given $q<2$, by Young's inequality we may estimate

$$
|\nabla v|^{q} \leq \frac{|\nabla v|^{2}}{1+v^{2}}+\left(1+v^{2}\right)^{\frac{q}{2-q}} \leq \frac{|\nabla v|^{2}}{1+v^{2}}+C\left(q,\|f\|_{L^{1}(\Omega)}\right) \cdot e^{2 \pi v\|f\|_{L^{1}(\Omega)}^{-1} .}
$$

The desired bound now follows from (14) and (18).

Finally, recall Sobolev's embedding $W_{0}^{1,2 \alpha}(\Omega) \hookrightarrow L^{\infty}(\Omega)$ for any $\alpha>1$. More precisely we have

Theorem 2.4. There is a constant $\alpha_{0}=\alpha_{0}(\Omega)$ such that for any $\left.\left.\alpha \in\right] 1, \alpha_{0}\right]$, any $u \in W_{0}^{1,2 \alpha}(\Omega)$ there holds $u \in L^{\infty}(\Omega)$, and

$$
\|u\|_{L^{\infty}(\Omega)} \leq \frac{1}{\sqrt{\alpha-1}}\|u\|_{W_{0}^{1,2 \alpha}(\Omega)} .
$$

Proof. Extend $u \equiv 0$ on $\mathbb{R}^{2} \backslash \Omega$. Suppose $\sup _{\Omega}|u|$ is achieved at $x_{0} \in \Omega$. Shifting coordinates, we may assume $x_{0}=0$ and $u \equiv 0$ outside $B_{2 R_{0}}(0)$. Represent

$$
u(0)=\int_{B_{2 R_{0}}(0)} \nabla u \nabla G d x=\int_{\Omega} \nabla u \nabla G d x,
$$

where $G(x)=\frac{1}{2 \pi} \log \left(\frac{2 R_{0}}{|x|}\right)$. By Hölder's inequality then

$$
\begin{aligned}
\|u\|_{L^{\infty}(\Omega)} & =|u(0)| \leq \frac{1}{2 \pi}\left(\int_{\Omega}|\nabla u|^{2 \alpha} d x\right)^{\frac{1}{2 \alpha}}\left(\int_{B_{2 R_{0}}(0)}|x|^{-\frac{2 \alpha}{2 \alpha-1}} d x\right)^{\frac{2 \alpha-1}{2 \alpha}} \\
& \leq\|\nabla u\|_{L^{2 \alpha}(\Omega)}\left(\frac{2 \alpha-1}{2 \alpha-2}\left(2 R_{0}\right)^{\frac{2 \alpha-2}{2 \alpha-1}}\right)^{\frac{2 \alpha-1}{2 \alpha}}
\end{aligned}
$$

Since, as $\alpha \rightarrow 1$,

$$
\left(\frac{2 \alpha-1}{2}\left(2 R_{0}\right)^{\frac{2 \alpha-2}{2 \alpha-1}}\right)^{\frac{2 \alpha-1}{2 \alpha}} \rightarrow \frac{1}{\sqrt{2}}<1,(\alpha-1)^{\frac{\alpha-1}{2 \alpha}} \rightarrow 1,
$$

we obtain the desired bound

$$
\|u\|_{L^{\infty}(\Omega)} \leq \frac{1}{\sqrt{\alpha-1}}\|u\|_{W_{0}^{1,2 \alpha}(\Omega)}
$$

for $1<\alpha \leq \alpha_{0}(\Omega)$. 


\section{Approximate solutions}

In this section we give the proof of Theorem 1.2. Observe that for sufficiently small domains $\Omega$ any nontrivial solution $u \neq 0$ of (11) automatically will be positive. Indeed, let $\lambda_{1}=\lambda_{1}(\Omega)$ denote the first Dirichlet eigenvalue of the operator $-\Delta$ on $H_{0}^{1}(\Omega)$, characterized by

$$
\lambda_{1}=\inf _{0 \neq u \in H_{0}^{1}(\Omega)} \int_{\Omega}|\nabla u|^{2} d x / \int_{\Omega} u^{2} d x
$$

In view of the natural inclusion $H_{0}^{1}(\Omega) \hookrightarrow H_{0}^{1}\left(B_{R}(0)\right)$ and the natural scaling, for $\Omega \subset B_{R}(0)$ we have

$$
\lambda_{1}(\Omega) \geq \lambda_{1}\left(B_{R}(0)\right)=R^{-2} \lambda_{1}\left(B_{1}(0)\right) .
$$

Hence we may choose $R_{0}>0$ such that

$$
\lambda_{1}(\Omega) \geq \lambda_{1}\left(B_{R_{0}}(0)\right)>e=\sup _{s \leq 0} e^{\varphi(s)}
$$

for $\Omega \subset B_{R_{0}}(0)$.

Suppose $u \in W_{0}^{1,2 \alpha}(\Omega)$ solves (11) for some $\alpha>1$ and suppose, by contradiction, that $u \nsupseteq 0$. Multiplying (11) by the function $u_{-}=\min \{u, 0\} \in$ $W_{0}^{1,2 \alpha}(\Omega)$ and integrating by parts, we then find that

$$
\begin{gathered}
\int_{\Omega}\left|\nabla u_{-}\right|^{2} d x \leq \int_{\Omega}\left(1+|\nabla u|^{2}\right)^{\alpha-1}\left|\nabla u_{-}\right|^{2} d x=\int_{\Omega} u_{-} f(u) d x \\
\leq \sup _{s \leq 0} e^{\varphi(s)} \int_{\Omega}\left|u_{-}\right|^{2} d x<\lambda_{1}(\Omega) \int_{\Omega}\left|u_{-}\right|^{2} d x \leq \int_{\Omega}\left|\nabla u_{-}\right|^{2} d x .
\end{gathered}
$$

Hence, $u_{-} \equiv 0$ and therefore $u \geq 0$. Strict positivity then follows from the maximum principle; see for instance [8], Proposition 4.9.

In the following we always assume that $\Omega \subset B_{R_{0}}(0)$ satisfies (19); however, we reserve the freedom to decrease $R_{0}$ further, if necessary.

For $\alpha \geq 1$, consider the function $g_{\alpha}: W_{0}^{1,2 \alpha}(\Omega) \rightarrow \mathbb{R}$, given by

$$
g_{\alpha}(u)=\left\langle d E_{\alpha}(u), u\right\rangle=\int_{\Omega}\left(1+|\nabla u|^{2}\right)^{\alpha-1}|\nabla u|^{2} d x-\int_{\Omega} f(u) u d x,
$$

where $\langle\cdot, \cdot\rangle: W^{-1,2 \alpha}(\Omega) \times W_{0}^{1,2 \alpha}(\Omega) \rightarrow \mathbb{R}$ denotes duality pairing, and define the Nehari manifold

$$
M_{\alpha}=\left\{u \in W_{0}^{1,2 \alpha}(\Omega) \backslash\{0\} ; g_{\alpha}(u)=0\right\} .
$$




\subsection{Regularity of $M_{\alpha}$}

Observe that $E_{\alpha}$ and $g_{\alpha}$ are of class $C^{1,1}$ with

$$
\begin{aligned}
\left\langle d g_{\alpha}(u), v\right\rangle= & 2 \int_{\Omega}\left(1+\alpha|\nabla u|^{2}\right)\left(1+|\nabla u|^{2}\right)^{\alpha-2} \nabla u \nabla v d x \\
& -\int_{\Omega}\left(2+\varphi^{\prime}(u) u\right) f(u) v d x .
\end{aligned}
$$

Lemma 3.1. There exists a constant $C_{0}>0$ such that for $u \in M_{\alpha}$ with $\alpha \geq 1$ there holds

$$
\|u\|_{H_{0}^{1}(\Omega)}^{2} \geq C_{0} .
$$

Proof. Note that $f^{\prime}(0)=1$. By (19) then, with error $o(1) \rightarrow 0$ as $\|u\|_{H_{0}^{1}(\Omega)} \rightarrow 0$, we have

$$
\begin{aligned}
\int_{\Omega} f(u) u d x & \leq f^{\prime}(0) \int_{\Omega} u^{2} d x+o(1)\|u\|_{H_{0}^{1}(\Omega)}^{2} \\
& \leq\left(\lambda_{1}(\Omega)^{-1}+o(1)\right)\|u\|_{H_{0}^{1}(\Omega)}^{2}<\|u\|_{H_{0}^{1}(\Omega)}^{2} \\
& \leq \int_{\Omega}\left(1+|\nabla u|^{2}\right)^{\alpha-1}|\nabla u|^{2} d x
\end{aligned}
$$

for any $\alpha \geq 1$ and any $u \in W_{0}^{1,2 \alpha}(\Omega) \hookrightarrow H_{0}^{1}(\Omega)$, if $0<\|u\|_{H_{0}^{1}(\Omega)}<C_{0}$ is sufficiently small. Hence, $g_{\alpha}(u)>0$ for any $\alpha$ and any such $u$.

Lemma 3.2. For $u \in M_{\alpha}$ and $1 \leq \alpha \leq 2$ there holds

$\left\langle d g_{\alpha}(u), u\right\rangle \leq \int_{\Omega}\left[2\left(1+|\nabla u|^{2}\right)^{\alpha-1}|\nabla u|^{2}-\varphi^{\prime}(u) u^{2} f(u)\right] d x \leq-\frac{1}{2} C_{0}<0$,

provided $R_{0}>0$ is sufficiently small.

Proof. Compute, using that $g_{\alpha}(u)=0$,

$$
\begin{aligned}
& \left\langle d g_{\alpha}(u), u\right\rangle \\
& =2 \int_{\Omega}\left(1+\alpha|\nabla u|^{2}\right)\left(1+|\nabla u|^{2}\right)^{\alpha-2}|\nabla u|^{2} d x-\int_{\Omega}\left(2+\varphi^{\prime}(u) u\right) f(u) u d x \\
& \leq 2 \alpha \int_{\Omega}\left(1+|\nabla u|^{2}\right)^{\alpha-1}|\nabla u|^{2} d x-\int_{\Omega}\left(2+\varphi^{\prime}(u) u\right) f(u) u d x \\
& =\int_{\Omega}\left(2(\alpha-1)-\varphi^{\prime}(u) u\right) f(u) u d x \leq \int_{\Omega}\left(2-\varphi^{\prime}(u) u\right) f(u) u d x=: I,
\end{aligned}
$$

and the first inequality follows. 
Let $s_{0}>0$ be chosen such that $\varphi^{\prime}(s) s \geq 3$ for $s \geq s_{0}$, which is possible by assumption (9). Then, by Lemma 3.1 and using our assumptions on $\varphi$ together with the equation $g_{\alpha}(u)=0$, we can estimate the latter

$$
\begin{aligned}
I & \leq 3 \int_{\left\{x \in \Omega ; u(x) \leq s_{0}\right\}} f(u) u d x-\int_{\left\{x \in \Omega ; u(x) \geq s_{0}\right\}} f(u) u d x \\
& =4 \int_{\left\{x \in \Omega ; u(x) \leq s_{0}\right\}} f(u) u d x-\int_{\Omega} f(u) u d x \\
& \leq C_{1} \int_{\Omega} u^{2} d x-\int_{\Omega}\left(1+|\nabla u|^{2}\right)^{\alpha-1}|\nabla u|^{2} d x \\
& \leq\left(C_{1} \lambda_{1}(\Omega)^{-1}-1\right)\|u\|_{H_{0}^{1}(\Omega)}^{2},
\end{aligned}
$$

where $C_{1}=4 \sup _{s \leq s_{0}} e^{\varphi(s)}$. By (19) now for sufficiently small $R_{0}>0$ we can estimate

$$
C_{1} \lambda_{1}(\Omega)^{-1} \leq \frac{1}{2}
$$

and therefore

$$
I \leq-\frac{1}{2}\|u\|_{H_{0}^{1}(\Omega)}^{2} \leq-\frac{1}{2} C_{0}
$$

on account of Lemma 3.1.

We conclude that for $1 \leq \alpha \leq 2$ and $\Omega \subset B_{R_{0}}(0)$ the set $M_{\alpha}$ is a complete $C^{1,1}$-submanifold of $W_{0}^{1,2 \alpha}(\Omega)$.

\subsection{Palais-Smale condition}

Using Lemma 3.2, for $1<\alpha \leq 2$ we can compare $d E_{\alpha}$ and the derivative of $E_{\left.\alpha\right|_{M_{\alpha}}}$ with norm

$$
\left\|d E_{\alpha}(u)\right\|_{T_{u}^{*} M_{\alpha}}=\sup \left\{\left\langle d E_{\alpha}(u), v\right\rangle ; v \in T_{u} M_{\alpha},\|v\|_{W_{0}^{1,2 \alpha}} \leq 1\right\} .
$$

Lemma 3.3. For any $\alpha \in] 1,2]$, any $\beta \in \mathbb{R}$ there is a constant $C=C(\alpha, \beta)$ such that

$$
\left\|d E_{\alpha}(u)\right\|_{W^{-1,2 \alpha}(\Omega)} \leq C\left\|d E_{\alpha}(u)\right\|_{T_{u}^{*} M_{\alpha}}
$$

for all $u \in M_{\alpha}$ with $\|u\|_{W_{0}^{1,2 \alpha}(\Omega)} \leq \beta$. The constant $C=C(\alpha, \beta)$ may be chosen to be non-increasing in $\alpha$ and non-decreasing in $\beta$.

Proof. Fix $u \in M_{\alpha}$ with $\|u\|_{W_{0}^{1,2 \alpha}(\Omega)} \leq \beta$. Given $w \in W_{0}^{1,2 \alpha}(\Omega)$ with $\|w\|_{W_{0}^{1,2 \alpha}(\Omega)} \leq 1$, decompose $w=\gamma u+v$ where $v \in T_{u} M_{\alpha}$; that is, where 
$v \in W_{0}^{1,2 \alpha}(\Omega)$ satisfies $\left\langle d g_{\alpha}(u), v\right\rangle=0$. Note that by Sobolev's embed$\operatorname{ding} W_{0}^{1,2 \alpha}(\Omega) \hookrightarrow C^{0}(\bar{\Omega})$ there holds $u, w \in L^{\infty}(\Omega)$ with $\|u\|_{L^{\infty}(\Omega)} \leq C$, $\|w\|_{L^{\infty}(\Omega)} \leq C$. Thus, by Young's inequality we can estimate

$$
\begin{aligned}
\left|\left\langle d g_{\alpha}(u), w\right\rangle\right| \leq & 2 \int_{\Omega}\left(1+\alpha|\nabla u|^{2}\right)\left(1+|\nabla u|^{2}\right)^{\alpha-2}|\nabla u||\nabla w| d x \\
& +\int_{\Omega}\left|2+\varphi^{\prime}(u) u\right||f(u)||w| d x \\
\leq & 2 \int_{\Omega}\left(\left(1+\alpha|\nabla u|^{2}\right)^{\alpha}+|\nabla w|^{2 \alpha}\right) d x+C \leq C
\end{aligned}
$$

with uniform constants $C=C(\alpha, \beta)$. By Lemma 3.2 then

$$
|\gamma|=\left|\frac{\left\langle d g_{\alpha}(u), w\right\rangle}{\left\langle d g_{\alpha}(u), u\right\rangle}\right| \leq C
$$

and therefore also $\|v\|_{W_{0}^{1,2 \alpha}(\Omega)} \leq C$.

Hence

$$
\begin{aligned}
\left\langle d E_{\alpha}(u), w\right\rangle=\left\langle d E_{\alpha}(u), v\right\rangle & \leq\left\|d E_{\alpha}(u)\right\|_{T_{u}^{*} M_{\alpha}}\|v\|_{W_{0}^{1,2 \alpha}(\Omega)} \\
& \leq C\left\|d E_{\alpha}(u)\right\|_{T_{u}^{*} M_{\alpha}},
\end{aligned}
$$

and the claim follows by taking the supremum with respect to $w$.

Inspection of the proof shows that $C=C(\alpha, \beta)$ may be chosen to monotonically depend on $\alpha$ and $\beta$, as asserted.

The functional $E_{\alpha}$ is coercive on $M_{\alpha}$ for all $\alpha \geq 1$ and for $\alpha>1$ satisfies the Palais-Smale condition on $M_{\alpha}$.

Lemma 3.4. $i)$ There exists a uniform constant $C$ depending only on $f$ such that for any $\alpha \geq 1$ and any $u \in M_{\alpha}$ there holds

$$
\|u\|_{W_{0}^{1,2 \alpha}(\Omega)}^{2 \alpha} \leq 4 \alpha E_{\alpha}(u)+C .
$$

ii) If $\alpha>1$ and if $\left(u^{(n)}\right) \subset M_{\alpha}$ satisfies

$$
E_{\alpha}\left(u^{(n)}\right) \rightarrow \beta,\left\|d E_{\alpha}\left(u^{(n)}\right)\right\|_{T_{u^{(n)}}^{*} M_{\alpha}} \rightarrow 0(n \rightarrow \infty),
$$

then a subsequence $\left(u^{(n)}\right)$ converges strongly to a solution $u \in M_{\alpha}$ of (11). 
Proof. i) Using exponential growth (10) and the fact that $g_{\alpha}(u)=0$, for $u \in M_{\alpha}$ we obtain

$$
\begin{aligned}
2 \alpha E_{\alpha}(u) & =2 \alpha E_{\alpha}(u)-g_{\alpha}(u) \\
& =\int_{\Omega}\left[\left(1+|\nabla u|^{2}\right)^{\alpha-1}-1+f(u) u-2 \alpha F(u)\right] d x \\
& \geq \int_{\Omega}(f(u) u-2 \alpha F(u)) d x \geq \frac{1}{2} \int_{\Omega} f(u) u d x-C \\
& =\frac{1}{2} \int_{\Omega}\left(1+|\nabla u|^{2}\right)^{\alpha-1}|\nabla u|^{2} d x-C \geq \frac{1}{2}\|u\|_{W_{0}^{1,2 \alpha}(\Omega)}^{2 \alpha}-C .
\end{aligned}
$$

ii) By part i), the sequence $\left(u^{(n)}\right)$ is bounded. Moreover, by Lemma 3.3 it follows that $d E_{\alpha}\left(u^{(n)}\right) \rightarrow 0$ in $W^{-1,2 \alpha}(\Omega)$. By compactness of the embedding $W_{0}^{1,2 \alpha}(\Omega) \hookrightarrow C^{0}(\bar{\Omega})$, we may assume that $u^{(n)} \rightarrow u, f\left(u^{(n)}\right) \rightarrow f(u)$ uniformly as $n \rightarrow \infty$. But then, with error $o(1) \rightarrow 0$ as $n \rightarrow \infty$,

$$
\begin{aligned}
o(1) & =\left\langle d E_{\alpha}\left(u^{(n)}\right), u^{(n)}-u\right\rangle \\
& =\int_{\Omega}\left(1+\left|\nabla u^{(n)}\right|^{2}\right)^{\alpha-1} \nabla u^{(n)} \cdot\left(\nabla u^{(n)}-\nabla u\right) d x+o(1) .
\end{aligned}
$$

Now observe uniform convexity of the function $\mathbb{R}^{2} \ni z \mapsto \frac{\left(1+|z|^{2}\right)^{\alpha}}{2 \alpha}=: e_{\alpha}(z)$ in the sense that

$$
e_{\alpha}(z) \geq e_{\alpha}\left(z_{0}\right)+d e_{\alpha}\left(z_{0}\right)\left(z-z_{0}\right)+\frac{1}{2}\left|z-z_{0}\right|^{2} .
$$

Applying this relation with $z=\nabla u(x), z_{0}=\nabla u^{(n)}(x)$, and integrating, we obtain

$$
o(1) \geq \int_{\Omega} \frac{\left(1+\left|\nabla u^{(n)}\right|^{2}\right)^{\alpha}-\left(1+|\nabla u|^{2}\right)^{\alpha}}{2 \alpha} d x+\frac{1}{2} \int_{\Omega}\left|\nabla\left(u^{(n)}-u\right)\right|^{2} d x,
$$

and $u^{(n)} \rightarrow u$ in $W_{0}^{1,2 \alpha}(\Omega)$, as claimed.

To proceed, we make the following technical assumption:

There is no solution $0<u \in H_{0}^{1}(\Omega)$ of (1) with energy $E(u) \leq \frac{1}{2}$.

Clearly, should assumption (21) not hold, the proof of Theorem 1.2 will be complete.

Concerning the macroscopic behavior of "Palais-Smale sequences" as $\alpha \searrow 1$ then we have: 
Lemma 3.5. For a sequence $\alpha \searrow 1$ let $u_{\alpha} \in M_{\alpha}$ satisfy $\left\|d E_{\alpha}\left(u_{\alpha}\right)\right\|_{T_{u_{\alpha}}^{*} M_{\alpha}}$ $\rightarrow 0$ and $E_{\alpha}\left(u_{\alpha}\right) \rightarrow \beta<1$. Assume condition (21) holds. Then either i) a subsequence $u_{\alpha} \rightarrow u$ in $H_{0}^{1}(\Omega)$, where $u$ solves (1), (8) with $E(u)=\beta$, or ii) $\beta \geq \frac{1}{2}$ and $u_{\alpha} \rightarrow 0$ weakly in $H_{0}^{1}(\Omega), f\left(u_{\alpha}\right) \rightarrow 0, F\left(u_{\alpha}\right) \rightarrow 0$ in $L^{1}(\Omega)$ and there exists a point $x_{0} \in \bar{\Omega}$ and a number $m_{0} \in[1,2 \beta]$ such that, as $\alpha \searrow 1$,

$$
\left|\nabla u_{\alpha}\right|^{2 \alpha} d x \rightarrow 2 \beta \delta_{x_{0}},\left|\nabla u_{\alpha}\right|^{2} d x \rightarrow m_{0} \delta_{x_{0}}, u_{\alpha} f\left(u_{\alpha}\right) d x \rightarrow 2 \beta \delta_{x_{0}}
$$

weakly in the sense of measures.

Proof. By Lemma 3.4 we may assume that $u_{\alpha} \rightarrow u$ weakly in $H_{0}^{1}(\Omega)$ as $\alpha \rightarrow 1$ and pointwise almost everywhere. Moreover, the normalization $g_{\alpha}\left(u_{\alpha}\right)=0$ implies the uniform bound

$$
\int_{\Omega} u_{\alpha} f\left(u_{\alpha}\right) d x=\int_{\Omega}\left(1+\left|\nabla u_{\alpha}\right|^{2}\right)^{\alpha-1}\left|\nabla u_{\alpha}\right|^{2} d x \leq C .
$$

From exponential growth (10) we thus deduce that the families $\left(F\left(u_{\alpha}\right)\right)_{\alpha>1}$, $\left(f\left(u_{\alpha}\right)\right)_{\alpha>1}$ are equi-integrable. Vitali's theorem then implies convergence $F\left(u_{\alpha}\right) \rightarrow F(u), f\left(u_{\alpha}\right) \rightarrow f(u)$ in $L^{1}(\Omega)$. Since also $\left(1+\left|\nabla u_{\alpha}\right|^{2}\right)^{\alpha-1} \rightarrow 1$ in $L^{2}(\Omega)$ as $\alpha \rightarrow 1$, in particular, we conclude that $u$ is a weak solution of (1), (8).

Indeed, given $w \in C^{\infty}(\bar{\Omega}) \cap H_{0}^{1}(\Omega)$, for any $\alpha>1$ decompose $w=$ $\gamma_{\alpha} u_{\alpha}+v$, where $\left\langle d g_{\alpha}\left(u_{\alpha}\right), v\right\rangle=0$. In view of Lemma 3.2 we can estimate

$$
\begin{aligned}
\gamma_{\alpha}= & \frac{\left\langle d g_{\alpha}\left(u_{\alpha}\right), w\right\rangle}{\left\langle d g_{\alpha}\left(u_{\alpha}\right), u_{\alpha}\right\rangle} \leq C\|w\|_{C^{1}(\bar{\Omega})} \int_{\Omega}\left(1+\left|\nabla u_{\alpha}\right|^{2 \alpha-1}\right) d x \\
& +\|w\|_{L^{\infty}} \int_{\Omega}\left(\left|f^{\prime}\left(u_{\alpha}\right) u_{\alpha}\right|+\left|f\left(u_{\alpha}\right)\right|\right) d x /\left|\left\langle d g_{\alpha}\left(u_{\alpha}\right), u_{\alpha}\right\rangle\right| \\
\leq & C+C \int_{\Omega}\left(1+u_{\alpha}^{2}\right)\left|f\left(u_{\alpha}\right)\right| d x / \int_{\Omega} \varphi^{\prime}\left(u_{\alpha}\right) u_{\alpha}^{2} f\left(u_{\alpha}\right) d x \leq C .
\end{aligned}
$$

We conclude that $\|v\|_{W_{0}^{1,2 \alpha}(\Omega)} \leq C$, uniformly in $\alpha>1$. Hence

$$
\left\langle d E_{\alpha}\left(u_{\alpha}\right), w\right\rangle=\left\langle d E_{\alpha}\left(u_{\alpha}\right), v\right\rangle \leq C\left\|d E_{\alpha}\left(u_{\alpha}\right)\right\|_{T_{u_{\alpha}}^{*} M_{\alpha}} \rightarrow 0
$$

as $\alpha \rightarrow 1$.

It follows that

$$
\begin{aligned}
\int_{\Omega}(\nabla u \nabla w-f(u) w) d x & =\lim _{\alpha \rightarrow 1} \int_{\Omega}\left(\left(1+\left|\nabla u_{\alpha}\right|^{2}\right)^{\alpha-1} \nabla u_{\alpha} \nabla w-f\left(u_{\alpha}\right) w\right) d x \\
& =\lim _{\alpha \rightarrow 1}\left\langle d E_{\alpha}\left(u_{\alpha}\right), w\right\rangle=0 ;
\end{aligned}
$$

that is, $u$ solves (1), as claimed. 
In particular, $u$ is smooth. Thus, $w=u$ is admissible above and with error $o(1) \rightarrow 0$ as $\alpha \rightarrow 1$, from (20) we have

$$
\begin{aligned}
o(1) & =\left\langle d E_{\alpha}\left(u_{\alpha}\right), u_{\alpha}-u\right\rangle \\
& =\int_{\Omega}\left(1+\left|\nabla u_{\alpha}\right|^{2}\right)^{\alpha-1} \nabla u_{\alpha} \nabla\left(u_{\alpha}-u\right)-f\left(u_{\alpha}\right)\left(u_{\alpha}-u\right) d x \\
& \geq \frac{1}{2} \int_{\Omega}\left|\nabla\left(u_{\alpha}-u\right)\right|^{2} d x-\int_{\Omega} f\left(u_{\alpha}\right)\left(u_{\alpha}-u\right) d x+o(1) .
\end{aligned}
$$

Moreover, we find

$\beta=E_{\alpha}\left(u_{\alpha}\right)+o(1) \geq E\left(u_{\alpha}\right)+o(1)=E(u)+\frac{1}{2} \int_{\Omega}\left|\nabla\left(u_{\alpha}-u\right)\right|^{2} d x+o(1)$.

Let $\left|\nabla\left(u_{\alpha}-u\right)\right|^{2} d x \rightarrow \mu$ in the sense of measures. Note that $\mu$ has at most finitely many atoms of mass $m_{i} \geq 1$ at points $x_{i} \in \bar{\Omega}, 0 \leq i<L$. Since $E(u) \geq 0$ on account of (21), we conclude that

$$
1>\beta \geq E(u)+\frac{1}{2} \int_{\Omega} d \mu \geq \frac{L}{2}
$$

that is, $L \leq 1$.

Let $x_{0} \in \bar{\Omega}$. Suppose there is $r=r_{x_{0}}>0$ such that

$$
\limsup _{\alpha \rightarrow 1} \int_{B_{r}\left(x_{0}\right)}\left|\nabla u_{\alpha}\right|^{2} d x<1
$$

For $r_{0} \leq \min \left\{\exp (-e), r_{x_{0}}\right\}$ define the cut-off function

$$
\psi(r)=\min \left\{1, \log \log \log \left(\frac{1}{r}\right)-\log \log \log \left(\frac{1}{r_{0}}\right)\right\},
$$

if $r \leq r_{0}, \psi(r)=0$ else. Note that $\psi(r)=1$ for $r \leq r_{1}=\exp \left(-\left(\log \frac{1}{r_{0}}\right)^{e}\right)$.

Computing

$$
\left|\psi^{\prime}(r)\right|^{2}=\frac{1}{r^{2}\left(\log \left(\frac{1}{r}\right) \log \log \left(\frac{1}{r}\right)\right)^{2}}, \text { if } r_{1} \leq r \leq r_{0},
$$

moreover, we easily see that

$$
\begin{aligned}
& \int_{0}^{r_{0}}\left|\psi^{\prime}(r)\right|^{2}\left(1+\log \left(1+\left|\psi^{\prime}(r)\right|^{2}\right)\right) r d r \\
& \quad \leq C \int_{r_{1}}^{r_{0}} \frac{d r}{r \log \left(\frac{1}{r}\right)\left(\log \log \left(\frac{1}{r}\right)\right)^{2}} \leq \frac{C}{\log \log \left(\frac{1}{r_{0}}\right)} \rightarrow 0
\end{aligned}
$$

as $r_{0} \rightarrow 0$. 
Given $x_{0} \in \Omega, r_{0}>0$ as above, then let

$$
v_{\alpha}(x)=\psi\left(\left|x-x_{0}\right|\right) u_{\alpha}(x) \in H_{0}^{1}(\Omega) .
$$

Observe that by Young's inequality for any $\varepsilon>0$ with a constant $C(\varepsilon)>1$ we can bound

$$
\begin{aligned}
\left|\nabla v_{\alpha}\right|^{2} & \leq\left|\nabla u_{\alpha}\right|^{2}+2|\nabla \psi| \psi\left|\nabla u_{\alpha}\right| u_{\alpha}+u_{\alpha}^{2}|\nabla \psi|^{2} \\
& \leq(1+\varepsilon)\left|\nabla u_{\alpha}\right|^{2}+C(\varepsilon) u_{\alpha}^{2}|\nabla \psi|^{2} .
\end{aligned}
$$

Define

$$
g(t)=\sup _{s>0}\left\{s^{2} t-s f(s)\right\}
$$

and note that for any $t>1$ we have

$$
g(t)=\frac{1}{2} \varphi^{\prime}(s) s^{2} f(s)_{\left.\right|_{2 s t=2 f(s)+\varphi^{\prime}(s) s f(s)}} \leq s^{2} t_{\left.\right|_{t=\frac{1}{2} \varphi^{\prime}(s) f(s)}} \leq C t \log (1+t) .
$$

Then for any $\varepsilon>0$ and any $0<s, t<\infty$ we can estimate

$$
\varepsilon s^{2} t \leq \varepsilon s f(s)+C \varepsilon t \log (1+t) .
$$

Applying this result with $s=u_{\alpha}, t=C(\varepsilon) \varepsilon^{-1}|\nabla \psi|^{2}$, we find that

$$
C(\varepsilon) u_{\alpha}^{2}|\nabla \psi|^{2} d x \leq \varepsilon u_{\alpha} f_{k}\left(u_{\alpha}\right)+C(\varepsilon)|\nabla \psi|^{2}\left(1+\log \left(1+|\nabla \psi|^{2}\right)\right)
$$

and hence

$$
\begin{gathered}
\int_{B_{r_{0}}\left(x_{0}\right)}\left|\nabla v_{\alpha}\right|^{2} d x \leq(1+\varepsilon) \int_{B_{r_{0}}\left(x_{0}\right)}\left|\nabla u_{\alpha}\right|^{2} d x+\varepsilon \int_{B_{r_{0}}\left(x_{0}\right)} u_{\alpha} f_{k}\left(u_{\alpha}\right) d x \\
+C(\varepsilon) \int_{B_{r_{0}}\left(x_{0}\right)}|\nabla \psi|^{2}\left(1+\log \left(1+|\nabla \psi|^{2}\right)\right) d x \leq C<1,
\end{gathered}
$$

if we first choose $\varepsilon>0$ and then $r_{0} \leq r_{x_{0}}$ sufficiently small.

By the Moser-Trudinger inequality, applied to $v_{\alpha} \in H_{0}^{1}(\Omega)$, we then conclude that the family $\left(e^{4 \pi v_{\alpha}^{2}}\right)_{k \in \mathbb{N}}$ is bounded in $L^{p}(\Omega)$ for some $p>1$. Hence also the functions $f\left(u_{\alpha}\right)$ are bounded in $L^{q}\left(B_{r_{1}}\left(x_{0}\right)\right)$ for some $q>1$, where $r_{1}=\exp \left(-\left(\log \left(\frac{1}{r_{0}}\right)\right)^{e}\right)$.

In particular, if $L=0$, upon covering $\bar{\Omega}$ with finitely many such balls $B_{r_{i}}\left(x_{i}\right)$, from pointwise convergence $u_{\alpha} \rightarrow u$ we then conclude that $f\left(u_{\alpha}\right) \rightarrow f(u)$ strongly in $H^{-1}(\Omega)$, and hence from (22) that $u_{\alpha} \rightarrow u$ in $H_{0}^{1}(\Omega)$.

Thus it remains the case $L=1$. Observe that necessarily $\beta \geq \frac{1}{2}$ in this case. Moreover, since $E(u) \leq \beta-\frac{1}{2}<\frac{1}{2}$, our assumption (21) implies that $u \equiv 0$. Given $r>0$, we may cover $\Omega \backslash B_{r}\left(x_{0}\right)$ by finitely many balls $B_{r_{i}}\left(x_{i}\right)$ as above to conclude that $\left(f\left(u_{\alpha}\right)\right)_{\alpha>1}$ is bounded in $L^{q}$ on $\Omega \backslash B_{r}\left(x_{0}\right)$ 
for some $q>1$. Fixing a cut-off function $\psi \in C_{0}^{\infty}\left(B_{2 r}\left(x_{0}\right)\right)$ such that $0 \leq \psi \leq 1, \psi=1$ in $B_{r}\left(x_{0}\right)$, then

$$
u_{\alpha}(1-\psi) \rightarrow 0 \text { weakly in } H_{0}^{1}\left(\Omega \backslash B_{r}\left(x_{0}\right)\right)
$$

and hence

$$
\int_{\Omega} f\left(u_{\alpha}\right) u_{\alpha}(1-\psi) d x \rightarrow 0(\alpha \rightarrow 1) .
$$

Also define, for $\alpha>1$ and $\psi$ as above

$$
w_{\alpha}=\psi u_{\alpha} \in W_{0}^{1,2 \alpha}(\Omega) .
$$

Decompose $w_{\alpha}=\gamma_{\alpha} u_{\alpha}+v_{\alpha}$, where $\gamma_{\alpha} \in \mathbb{R}$ and $\left\langle d g_{\alpha}\left(u_{\alpha}\right), v_{\alpha}\right\rangle=0$.

Similar to before, using Lemmas 3.2 and 3.4 we estimate

$$
\begin{aligned}
\gamma_{\alpha}= & \frac{\left\langle d g_{\alpha}\left(u_{\alpha}\right), w_{\alpha}\right\rangle}{\left\langle d g_{\alpha}\left(u_{\alpha}\right), u_{\alpha}\right\rangle} \leq C \int_{\Omega}\left(1+\left|\nabla u_{\alpha}\right|^{2}\right)^{(\alpha-1)}\left|\nabla u_{\alpha}\right|\left|\nabla\left(\psi u_{\alpha}\right)\right| d x \\
& +\int_{\Omega}\left(\left|f^{\prime}\left(u_{\alpha}\right)\right| u_{\alpha}^{2}+\left|f\left(u_{\alpha}\right) u_{\alpha}\right|\right) \psi d x /\left|\left\langle d g_{\alpha}\left(u_{\alpha}\right), u_{\alpha}\right\rangle\right| \leq C .
\end{aligned}
$$

Hence, $\left\|v_{\alpha}\right\|_{W_{0}^{1,2 \alpha}(\Omega)} \leq C$. Also using that $u_{\alpha} \in M_{\alpha}$, with error $o(1) \rightarrow 0$ as $\alpha \rightarrow 1$, we obtain

$$
\begin{aligned}
o(1) & =\left\langle d E_{\alpha}\left(u_{\alpha}\right), u_{\alpha}-w_{\alpha}\right\rangle=\left\langle d E_{\alpha}\left(u_{\alpha}\right),(1-\psi) u_{\alpha}\right\rangle \\
& =\int_{\Omega}\left(\left(1+\left|\nabla u_{\alpha}\right|^{2}\right)^{\alpha-1}\left|\nabla u_{\alpha}\right|^{2}(1-\psi)-f\left(u_{\alpha}\right) u_{\alpha}(1-\psi)\right) d x+o(1) \\
& \geq \int_{\Omega}\left|\nabla u_{\alpha}\right|^{2 \alpha}(1-\psi) d x+o(1) \\
& \geq \int_{\Omega \backslash B_{2 r}\left(x_{0}\right)}\left|\nabla u_{\alpha}\right|^{2 \alpha} d x+o(1) .
\end{aligned}
$$

Since $r>0$ was arbitrary, we conclude that $\left|\nabla u_{\alpha}\right|^{2 \alpha} \rightarrow 0, u_{\alpha} f\left(u_{\alpha}\right) \rightarrow 0$ in $L_{\text {loc }}^{1}\left(\Omega \backslash\left\{x_{0}\right\}\right)$, as claimed.

From the identity, with error $o(1) \rightarrow 0$ as $\alpha \rightarrow 1$,

$$
\begin{aligned}
\beta=E_{\alpha}\left(u_{\alpha}\right)+o(1) & =\int_{\Omega} \frac{\left(1+\left|\nabla u_{\alpha}\right|^{2}\right)^{\alpha}-1}{2 \alpha} d x+o(1) \\
& =\frac{1}{2} \int_{\Omega}\left|\nabla u_{\alpha}\right|^{2 \alpha} d x+o(1)
\end{aligned}
$$

we then deduce that

$$
\left|\nabla u_{\alpha}\right|^{2 \alpha} d x \rightarrow 2 \beta \delta_{x_{0}}
$$

weakly in the sense of measures. 
Finally, from

$$
\begin{aligned}
0=g_{\alpha}\left(u_{\alpha}\right) & =\int_{\Omega}\left(\left(1+\left|\nabla u_{\alpha}\right|^{2}\right)^{\alpha-1}\left|\nabla u_{\alpha}\right|^{2}-f\left(u_{\alpha}\right) u_{\alpha}\right) d x \\
& =\int_{\Omega}\left(\left|\nabla u_{\alpha}\right|^{2 \alpha}-f\left(u_{\alpha}\right) u_{\alpha}\right) d x+o(1),
\end{aligned}
$$

we also conclude that

$$
f\left(u_{\alpha}\right) u_{\alpha} d x \rightarrow 2 \beta \delta_{x_{0}}
$$

as claimed.

\subsection{Minima}

Standard variational methods allow to deduce the existence of minimizers.

Lemma 3.6. For any $\alpha>1$ there is $u_{\alpha} \in M_{\alpha}$ such that $E_{\alpha}\left(u_{\alpha}\right)=\inf _{M_{\alpha}} E_{\alpha}$.

Proof. Fix $\alpha>1$ and let $\left(u^{(n)}\right)$ be a minimizing sequence in $M_{\alpha}$ satisfying

$$
E_{\alpha}\left(u^{(n)}\right) \rightarrow \inf _{M_{\alpha}} E_{\alpha}(n \rightarrow \infty) .
$$

By Ekeland's variational principle [13], or arguing as in the proof of Lemma 3.10, part i), below, we may assume that, in addition,

$$
\left\|d E_{\alpha}\left(u^{(n)}\right)\right\|_{T_{u^{(n)}}^{*} M_{\alpha}} \rightarrow 0 .
$$

But then Lemma 3.4 implies that a subsequence $u^{(n)} \rightarrow u$ strongly in $W_{0}^{1,2 \alpha}(\Omega)$. Hence, $u \in M_{\alpha}$ and $E_{\alpha}(u)=\inf _{M_{\alpha}} E_{\alpha}$, as desired.

\subsection{Saddle points}

Our next aim is to verify a "linking condition" in the sense of critical point theory for a suitable subset of $M_{\alpha}$. For $0<\rho<R$ let

$$
m_{\rho, R}(x)=\frac{1}{\sqrt{2 \pi}} \begin{cases}\sqrt{\log \left(\frac{R}{\rho}\right)}, & 0 \leq|x| \leq \rho, \\ \log \left(\frac{R}{r}\right) / \sqrt{\log \left(\frac{R}{\rho}\right)}, & \rho \leq|x|=r<R, \\ 0, & R \leq|x|,\end{cases}
$$

and for $x_{0} \in \mathbb{R}^{2}$ let $m_{\rho, R, x_{0}}(x)=m_{\rho, R}\left(x-x_{0}\right)$.

The same family of scaled and truncated Green's functions was considered by Moser [18].

Given $\left.R_{1} \in\right] 0, R_{0}$, fix $\left.R \in\right] 0, R_{1} / 4\left[\right.$ and let $0<R_{2}<R / 4,0<\rho<R$. Suitable such numbers $\rho$ and $R_{2}$ will be determined below. Observe the 
relations $0<R_{2}<4 R_{2}<R<4 R<R_{1}<R_{0}$. For each $R_{2}$, moreover, we let $\tau=\tau_{R_{2}} \in C_{0}^{\infty}\left(B_{2 R_{2}}(0)\right)$ be a cut-off function $0 \leq \tau \leq 1$ satisfying $\tau \equiv 1$ on $B_{R_{2}}(0)$ and such that $\tau \rightarrow 0$ in $H^{1}\left(\mathbb{R}^{2}\right)$ as $R_{2} \rightarrow 0$.

For $\left|x_{0}\right|=2 R, 0 \leq s<1$ then define

$$
v_{s, x_{0}}(x)=m_{(1-s) \rho, R, s x_{0}}(x)(1-\tau(x)) .
$$

Note that our assumptions about $R, R_{1}, R_{2}$, and $\tau$ imply that $0 \neq v_{s, x_{0}} \in$ $W_{0}^{1, \infty}(\Omega)$ for all $s$ and $x_{0}$, provided $B_{R_{1}} \backslash B_{R_{2}}(0) \subset \Omega$.

Lemma 3.7. For any s, $x_{0}, \alpha$ such that $0 \leq s<1,\left|x_{0}\right|=2 R, 1 \leq \alpha \leq 2$ there is a unique number $a_{s, x_{0}, \alpha}>0$ such that

$$
w_{s, x_{0}, \alpha}=a_{s, x_{0}, \alpha} v_{s, x_{0}} \in M_{\alpha} .
$$

The map $\left(s, x_{0}\right) \mapsto a_{s, x_{0}, \alpha}$ is of class $C^{1,1}$.

Proof. Fix $0 \leq s<1,\left|x_{0}\right|=2 R, \alpha \geq 1$ and let $v=v_{s, x_{0}}$. Then, with error $o(1) \rightarrow 0$ as $a \rightarrow 0$, by (19) we have

$$
\begin{aligned}
g_{\alpha}(a v) & =a^{2} \int_{\Omega}\left(1+a^{2}|\nabla v|^{2}\right)^{\alpha-1}|\nabla v|^{2} d x-a^{2} \int_{\Omega} v^{2} e^{\varphi(a v)} d x \\
& \geq a^{2}\left(\int_{\Omega}\left(|\nabla v|^{2}-v^{2}\right) d x+o(1)\right) \\
& \geq a^{2}\left(\left(1-\lambda_{1}(\Omega)^{-1}\right)\|v\|_{H_{0}^{1}}^{2}+o(1)\right) \\
& >0
\end{aligned}
$$

for small $a>0$.

On the other hand, for $a \geq 1$ clearly we can estimate

$$
g_{\alpha}(a v) \leq C_{1} a^{2 \alpha}-C_{2} a^{2} e^{C_{3} a^{2}}
$$

with constants $C_{j}=C_{j}(v)>0, j=1,2,3$. Thus $g_{\alpha}(a v)<0$ for large $a$ and there exists $\bar{a}>0$ such that $g_{\alpha}(\bar{a} v)=0$. By Lemma 3.2 and the implicit function theorem $1 \leq \alpha \leq 2$ this number $\bar{a}=a_{s, x_{0}, \alpha}$ is unique and of class $C^{1,1}$ with respect to $s$ and $x_{0}$.

Lemma 3.8. Given $\delta>0$, there exist numbers $\alpha_{\delta}>1, s_{\delta}<1$ such that there holds

$$
\sup _{\left|x_{0}\right|=2 R} E_{\alpha}\left(w_{s_{\delta}, x_{0}, \alpha}\right)<\frac{1}{2}+\delta, \sup _{0 \leq s \leq s_{\delta},\left|x_{0}\right|=2 R}\left|E_{\alpha}\left(w_{s, x_{0}, \alpha}\right)-E\left(w_{s, x_{0}, 1}\right)\right|<\delta
$$

uniformly for $1 \leq \alpha \leq \alpha_{\delta}$. The number $s_{\delta}$ is independent of $\left.\rho \in\right] 0, R[$ and $R_{2} ; \alpha_{\delta}$ may be chosen to be non-decreasing as a function of $\rho$ and $R_{2}$. 
Proof. Consider first the case $\alpha=1$. As shown in [2], [5], there holds

$$
E\left(w_{s, x_{0}, 1}\right) \rightarrow \frac{1}{2} \text { as } s \rightarrow 1,
$$

uniformly for all $x_{0} \in \partial B_{2 R}(0)$. Moreover, $\operatorname{supp}\left(m_{(1-s) \rho, R, s x_{0}}\right) \cap B_{2 R_{2}}(0)=\emptyset$ for $s<1$ sufficiently close to 1 . Thus, given $\delta>0$, we can find $s_{\delta}<1$ independent of $R_{2}$ and $\rho$ such that

$$
\sup _{\left|x_{0}\right|=2 R} E\left(w_{s_{\delta}, x_{0}, 1}\right) \leq \frac{1+\delta}{2} .
$$

But for any $\rho$ and $R_{2}$ the family $w_{s, x_{0}, \alpha}$ continuously depends on $\alpha$ in $W_{0}^{1, p}(\Omega)$ for any $p<\infty$, uniformly in $x_{0}$ and $0 \leq s \leq s_{\delta}$. In particular then, as $\alpha \rightarrow 1$,

$$
\sup _{0 \leq s \leq s,\left|x_{0}\right|=2 R}\left|E_{\alpha}\left(w_{s, x_{0}, \alpha}\right)-E\left(w_{s, x_{0}, 1}\right)\right| \rightarrow 0,
$$

and we can find $\alpha_{\delta}>1$, as desired.

Lemma 3.9. Under assumption (21) there holds $\lim _{\alpha \searrow 1} \inf _{M_{\alpha}} E_{\alpha}=\frac{1}{2}$.

Proof. The upper bound

$$
\limsup \inf _{\alpha \searrow 1} E_{M_{\alpha}} \leq \frac{1}{2}
$$

follows from Lemma 3.8. To prove the lower bound, suppose by contradiction that there exists a sequence $\alpha \searrow 1$ and corresponding functions $u_{\alpha} \in M_{\alpha}$ with

$$
E_{\alpha}\left(u_{\alpha}\right) \rightarrow \beta<\frac{1}{2}
$$

By Lemma 3.6 above we may assume that

$$
E_{\alpha}\left(u_{\alpha}\right)=\inf _{M_{\alpha}} E_{\alpha}
$$

and hence that $u_{\alpha}$ solves (11). Lemma 3.5 and assumption (21) then give $\beta \geq \frac{1}{2}$, contradicting our assumption.

Lemma 3.10. For a sequence $\alpha \searrow 1$ let $u_{\alpha} \in M_{\alpha}$ satisfy $E_{\alpha}\left(u_{\alpha}\right) \rightarrow \beta \leq \frac{1}{2}$, and suppose (21) holds. Then $\beta=\frac{1}{2}$ and there exists $x_{0} \in \bar{\Omega}$ such that for a subsequence $\alpha \rightarrow 1$ there holds $\left|\nabla u_{\alpha}\right|^{2} d x \rightarrow \delta_{x_{0}}$ weakly in the sense of measures. 
Proof. i) First assume that, in addition, there holds

$$
\left\|d E_{\alpha}\left(u_{\alpha}\right)\right\|_{T_{u_{\alpha}}^{*} M_{\alpha}} \rightarrow 0(\alpha \rightarrow 1) .
$$

Then the claim follows from Lemma 3.5.

ii) In the general case for $\alpha>1$ let $\Phi:[0,1] \times M_{\alpha} \rightarrow M_{\alpha}$ denote a pseudo-gradient flow for $E_{\alpha}$ on $M_{\alpha}$; see for instance [16]. Then for any $\alpha$, any $t \in[0,1]$ there holds

$$
E_{\alpha}\left(\Phi\left(t, u_{\alpha}\right)\right) \leq E_{\alpha}\left(u_{\alpha}\right)-\frac{t}{2} \inf _{0 \leq s \leq t}\left\|d E_{\alpha}\left(\Phi\left(s, u_{\alpha}\right)\right)\right\|_{T_{\Phi\left(s, u_{\alpha}\right)}^{*} M_{\alpha}}
$$

as well as

$$
\sup _{0 \leq s \leq t}\left\|\Phi\left(s, u_{\alpha}\right)-u_{\alpha}\right\|_{W_{0}^{1,2 \alpha}(\Omega)} \leq t .
$$

Now, for $\alpha \rightarrow 1$ either there exists $t_{\alpha} \rightarrow 0$ such that $v_{\alpha}=\Phi\left(t_{\alpha}, u_{\alpha}\right) \in M_{\alpha}$ satisfies $d E_{\alpha}\left(v_{\alpha}\right) \rightarrow 0$, or there is $t_{0}>0$ such that

$$
\varepsilon=\limsup _{\alpha \rightarrow 1} \inf _{0 \leq s \leq t_{0}}\left\|d E_{\alpha}\left(\Phi\left(s, u_{\alpha}\right)\right)\right\|_{T_{\Phi\left(s, u_{\alpha}\right)}^{*} M_{\alpha}}>0 .
$$

Choosing $t=t_{0}$ in (24) above and letting $w_{\alpha}=\Phi\left(t_{0}, u_{\alpha}\right) \in M_{\alpha}$, for suitable $\alpha \rightarrow 1$ with error $o(1) \rightarrow 0$ in the second case we obtain

$$
E_{\alpha}\left(w_{\alpha}\right) \leq E_{\alpha}\left(u_{\alpha}\right)-\frac{t_{0} \varepsilon}{2}+o(1)=\frac{1}{2}-\frac{t_{0} \varepsilon}{2}+o(1),
$$

contradicting the assertion of Lemma 3.9.

Hence only the first possibility is compatible with our assumptions. Observe that $v_{\alpha}$ also satisfies the estimate $E_{\alpha}\left(v_{\alpha}\right) \leq E_{\alpha}\left(u_{\alpha}\right)$ and $\left\|u_{\alpha}-v_{\alpha}\right\|_{W_{0}^{1,2 \alpha}(\Omega)} \leq t_{\alpha} \rightarrow 0$. By part i) it follows that for a subsequence $\left|\nabla v_{\alpha}\right|^{2} d x \rightarrow \delta_{x_{0}}$ weakly in the sense of measures as $\alpha \rightarrow 1$. But by Hölder's inequality, with error $o(1) \rightarrow 0$ as $\alpha \rightarrow 1$ we have

$$
\left\|u_{\alpha}-v_{\alpha}\right\|_{H_{0}^{1}(\Omega)} \leq(1+o(1))\left\|u_{\alpha}-v_{\alpha}\right\|_{W_{0}^{1,2 \alpha}(\Omega)} \rightarrow 0 .
$$

Hence the asserted convergence

$$
\left|\nabla u_{\alpha}\right|^{2} d x \rightarrow \delta_{x_{0}}(\alpha \rightarrow 1)
$$

also holds for a subsequence of the original sequence $\left(u_{\alpha}\right)$.

For $\delta>0,1 \leq \alpha \leq \alpha_{\delta}, s_{\delta}<1$ as defined in Lemma 3.8 let

$$
\Gamma_{\alpha}=\left\{\gamma \in C^{0}\left(\left[0, s_{\delta}\right] \times \partial B_{2 R}(0) ; M_{\alpha}\right) ; \gamma\left(s_{\delta}, x_{0}\right)=w_{s_{\delta}, x_{0}, \alpha} \text { for all } x_{0}\right\}
$$

and define

$$
\beta_{\alpha}=\inf _{\gamma \in \Gamma_{\alpha}} \sup \left\{E_{\alpha}\left(\gamma\left(s, x_{0}\right)\right) ; 0 \leq s \leq s_{\delta},\left|x_{0}\right|=2 R\right\} .
$$


Lemma 3.11. Suppose assumption (21) holds true. Given $0<R<4 R<$ $R_{1}<R_{0}$, there exist numbers $0<\rho<R, 0<R_{2}<R / 4$ such that for sufficiently small $\delta>0$ and $1 \leq \alpha \leq \alpha_{\delta}$ there holds

$$
\frac{1}{2}+2 \delta<\beta_{\alpha}<1-\delta,
$$

provided $B_{R_{1}} \backslash B_{R_{2}}(0) \subset \Omega \subset B_{R_{0}}(0), 0 \notin \bar{\Omega}$.

Proof. To obtain an upper bound on $\beta_{\alpha}$ it suffices to consider the map $\gamma\left(s, x_{0}\right)=w_{s, x_{0}, \alpha}$ as comparison map. Note that for $R_{2}=0, \alpha=1$ we have

$$
E\left(w_{s, x_{0}, 1}\right) \rightarrow \frac{1}{2} \text { as } \rho \rightarrow 0,
$$

uniformly in $0 \leq s \leq 1,\left|x_{0}\right|=2 R$. Hence we may choose $\rho>0$ such that

$$
\sup _{s, x_{0}} E\left(w_{s, x_{0}, 1}\right)<\frac{3}{4} .
$$

Since our cut-off functions $\tau=\tau_{R_{2}}$ converge to 1 in $H^{1}(\Omega)$ as $R_{2} \rightarrow 0$, for sufficiently small $\left.R_{2} \in\right] 0, R / 4[$ we then have

$$
\sup _{s, x_{0}} E\left(w_{s, x_{0}, 1}\right)<\frac{7}{8} .
$$

For $\delta<\frac{1}{16}$ and $1<\alpha \leq \alpha_{\delta}$ the upper bound $\beta_{\alpha}<1-\delta$ then follows from Lemma 3.8 above.

To obtain the lower bound we argue by contradiction. Let now $\rho, R_{2}$ be fixed as above and suppose that for some sequence $\alpha \rightarrow 1$ we have

$$
\lim _{\alpha \rightarrow 1} \beta_{\alpha}=\frac{1}{2} .
$$

Choose a corresponding sequence of maps $\gamma_{\alpha} \in \Gamma_{\alpha}$ satisfying

$$
\sup _{s, x_{0}} E_{\alpha}\left(\gamma_{\alpha}\left(s, x_{0}\right)\right) \rightarrow \frac{1}{2} .
$$

By Lemma 3.10, for any sequence

$$
u_{\alpha}=\gamma_{\alpha}\left(s, x_{0}\right), s=s(\alpha), x_{0}=x_{0}(\alpha)
$$

as $\alpha \rightarrow 1$ a subsequence satisfies

$$
\left|\nabla u_{\alpha}\right|^{2} d x \rightarrow \delta_{y}
$$


weakly in the sense of measures, where $y \in \bar{\Omega}$. In particular, as $\alpha \rightarrow 1$ we find $\operatorname{dist}\left(m\left(u_{\alpha}\right), \Omega\right) \rightarrow 0$, where

$$
m(u)=\frac{\int_{\Omega} x|\nabla u|^{2} d x}{\int_{\Omega}|\nabla u|^{2} d x} .
$$

Since $0 \notin \bar{\Omega}$, it follows that for sufficiently small $\alpha>1$ there holds

$$
\left|m\left(\gamma_{\alpha}\left(s, x_{0}\right)\right)\right| \geq m_{0}>0
$$

uniformly with respect to $0 \leq s \leq s_{\delta}$ and $x_{0} \in \partial B_{2 R}(0)$.

Identifying $\partial B_{2 R}(0)$ with $S^{1}$, we define a 2-parameter family of maps $h=h(\cdot ; \alpha, s): S^{1} \rightarrow S^{1}$ by letting

$$
h\left(x_{0} ; \alpha, s\right)=\frac{m\left(\gamma_{\alpha}\left(s, x_{0}\right)\right)}{\left|m\left(\gamma_{\alpha}\left(s, x_{0}\right)\right)\right|} .
$$

For any fixed $\alpha>1$ then $h\left(\cdot ; \alpha, s_{\delta}\right)$ is homotopic to $h(\cdot ; \alpha, 0) \equiv$ const. On the other hand, noting that $\gamma_{\alpha}\left(s_{\delta}, x_{0}\right)=w_{s_{\delta}, x_{0}, \alpha}=a_{s_{\delta}, x_{0}, \alpha} v_{s_{\delta}, x_{0}}$ by definition of $\Gamma_{\alpha}$, we see that $h\left(\cdot ; \alpha, s_{\delta}\right)=h\left(\cdot ; 1, s_{\delta}\right)$ for all $\alpha \geq 1$. The latter in turn is homotopic to the identity, as we see by varying $s \in\left[s_{\delta}, 1\right]$.

The contradiction proves that $\liminf _{\alpha \rightarrow 1} \beta_{\alpha}>\frac{1}{2}$ and $\delta>0$ can be found, as claimed.

The existence of critical points $u_{\alpha} \in M_{\alpha}$ of $E_{\alpha}$ with energies $E_{\alpha}\left(u_{\alpha}\right)=\beta_{\alpha}$, as claimed in Theorem 1.2, now is a consequence of standard methods in critical point theory; see for instance [23], Chap. II.

Considerably more work is required in order to obtain critical points $u_{\alpha}$ also satisfying condition (12).

\subsection{Monotonicity}

Observe that the map $\alpha \mapsto \frac{\left(1+s^{2}\right)^{\alpha}-1}{\alpha}$ is non-decreasing for $\alpha \geq 1$ and any $s \geq 0$. Indeed, its derivative at $\alpha$,

$$
h(s)=\frac{\left(\alpha \log \left(1+s^{2}\right)-1\right)\left(1+s^{2}\right)^{\alpha}+1}{\alpha^{2}}
$$

vanishes at $s=0$ and satisfies

$$
h^{\prime}(s)=2 s \log \left(1+s^{2}\right)\left(1+s^{2}\right)^{\alpha-1} \geq 0 .
$$

Thus, for any $u \in C^{\infty}(\bar{\Omega})$ also the map $\alpha \mapsto E_{\alpha}(u)$ is non-decreasing, with derivative

$$
\partial_{\alpha} E_{\alpha}(u)=\int_{\Omega} \frac{\left(\alpha \log \left(1+|\nabla u|^{2}\right)-1\right)\left(1+|\nabla u|^{2}\right)^{\alpha}+1}{\alpha^{2}} d x .
$$


Moreover, if we extend $E_{\alpha}$ to $H_{0}^{1}(\Omega)$ by letting $E_{\alpha}(u)=\infty$ for $u \in$ $H_{0}^{1}(\Omega) \backslash W_{0}^{1,2 \alpha}(\Omega)$, the same is true for arbitrary $u \in H_{0}^{1}(\Omega)$.

Similarly, the maps $\alpha \mapsto\left(1+s^{2}\right)^{\alpha-1} s^{2}$ and $\alpha \mapsto\left(1+\alpha s^{2}\right)\left(1+s^{2}\right)^{\alpha-2} s^{2}$ are non-decreasing for $\alpha \geq 1$ and any $s \geq 0$; therefore also the maps $\alpha \mapsto g_{\alpha}(u)$ and $\alpha \mapsto\left\langle d g_{\alpha}(u), u\right\rangle$ are non-decreasing for any $u \in H_{0}^{1}(\Omega)$.

Let $u \in M_{\alpha}$. By Lemma 3.2 the number $a=1$ is the unique zero of

$$
\frac{d}{d a} E_{\alpha}(a u)=\left\langle d E_{\alpha}(a u), u\right\rangle=a^{-1} g_{\alpha}(a u), a>0,
$$

and

$$
\frac{d^{2}}{d a^{2}} E_{\alpha}(a u)_{\mid a=1}=\left\langle d g_{\alpha}(u), u\right\rangle<0 .
$$

In particular, it follows that

$$
E_{\alpha}(u)=\max _{a>0} E_{\alpha}(a u) .
$$

By monotonicity of the map $\alpha \mapsto g_{\alpha}(u)$, for any $\alpha^{\prime} \leq \alpha$ there holds $g_{\alpha^{\prime}}(u) \leq 0$. On the other hand, recalling that $\varphi(0)=0, \lambda_{1}(\Omega) \geq e$, for sufficiently small $a>0$ we obtain that

$$
\begin{aligned}
g_{\alpha^{\prime}}(a u) & =a^{2} \int_{\Omega}\left[\left(1+a^{2}|\nabla u|^{2}\right)^{\alpha^{\prime}-1}|\nabla u|^{2}-e^{\varphi(a u)} u^{2}\right] d x \\
& \geq a^{2} \int\left(|\nabla u|^{2}-2 u^{2}\right) d x>0 .
\end{aligned}
$$

Hence there exists a unique number $\left.\left.a^{\prime}=a^{\prime}(u) \in\right] 0,1\right]$ so that $a^{\prime} u \in M_{\alpha^{\prime}}$.

By Lemma 3.2 and the implicit function theorem, moreover, the number $a^{\prime}=a^{\prime}(u)$ depends in $C^{1}$-fashion on $u$ in $W_{0}^{1,2 \alpha^{\prime}}(\Omega)$. Since $W_{0}^{1,2 \alpha}(\Omega) \hookrightarrow$ $W_{0}^{1,2 \alpha^{\prime}}(\Omega)$, we then conclude that the map $u \mapsto a^{\prime}(u) u=u^{\prime}$ defines a $C^{1}$-injection $i=i_{\alpha, \alpha^{\prime}}: M_{\alpha} \rightarrow M_{\alpha^{\prime}}$.

From monotonicity of the map $\alpha \mapsto E_{\alpha}(u)$ and (27), finally, we see that

$$
E_{\alpha^{\prime}}\left(u^{\prime}\right) \leq E_{\alpha}\left(u^{\prime}\right) \leq E_{\alpha}(u)
$$

for any $u \in M_{\alpha}$ and any $\alpha^{\prime} \leq \alpha$.

As an immediate consequence we obtain monotonicity of $\alpha \mapsto \beta_{\alpha}$.

Lemma 3.12. For $1 \leq \alpha^{\prime} \leq \alpha$ there holds $\beta_{\alpha^{\prime}} \leq \beta_{\alpha}$.

Proof. Composing a map $\gamma \in \Gamma_{\alpha}$ with $i_{\alpha, \alpha^{\prime}}=i$, we obtain a map $\gamma^{\prime}=$ $i \circ \gamma \in \Gamma_{\alpha^{\prime}}$ satisfying

$$
E_{\alpha^{\prime}}\left(\gamma^{\prime}\left(s, x_{0}\right)\right) \leq E_{\alpha}\left(\gamma\left(s, x_{0}\right)\right)
$$

for all $s$ and $x_{0}$, in view of (28). The claim easily follows. 
By Rademacher's theorem then the map $\alpha \mapsto \beta_{\alpha}$ is almost everywhere differentiable with differential $0 \leq \beta_{\alpha}^{\prime} \in L^{1}\left(\left[1, \alpha_{\delta}\right]\right)$. It follows that

$$
2 \Lambda:=\liminf _{\alpha \rightarrow 1}\left((\alpha-1) \log \frac{1}{\alpha-1} \cdot \beta_{\alpha}^{\prime}\right)=0 .
$$

Indeed, if we assume $\Lambda>0$, for small $A>1$ there results

$$
\int_{1}^{A} \beta_{\alpha}^{\prime} d \alpha \geq \Lambda \int_{0}^{A-1} \frac{d s}{s|\log s|}=\infty
$$

contradicting $\beta_{\alpha}^{\prime} \in L^{1}\left(\left[1, \alpha_{\delta}\right]\right)$.

Lemma 3.13. Let $\alpha>1$ be a point of differentiability of $\beta$ and let $\beta_{\alpha}^{\prime}=: B$. Then there exists a critical point $u_{\alpha} \in M_{\alpha}$ of $E_{\alpha}$ with energy $E_{\alpha}\left(u_{\alpha}\right)=\beta_{\alpha}$ and satisfying $\partial_{\alpha} E_{\alpha}\left(u_{\alpha}\right) \leq B+3$.

Proof. Fix a strictly decreasing sequence $\alpha_{k} \searrow \alpha(k \rightarrow \infty)$. For $k \in \mathbb{N}$ let $i_{k}=i_{\alpha_{k}, \alpha}: M_{\alpha_{k}} \rightarrow M_{\alpha}$. We consider paths $\gamma=\gamma_{k} \in \Gamma_{\alpha_{k}}$ such that

$$
\sup _{s, x_{0}} E_{\alpha_{k}}\left(\gamma\left(s, x_{0}\right)\right) \leq \beta_{\alpha_{k}}+\left(\alpha_{k}-\alpha\right) .
$$

Since $\alpha$ by assumption is a point of differentiability of $\beta$, for sufficiently large $\beta$ we may estimate

$$
\beta_{\alpha_{k}} \leq \beta_{\alpha}+(B+1)\left(\alpha_{k}-\alpha\right) .
$$

Moreover, $\gamma$ induces a map $\tilde{\gamma}=i_{k} \circ \gamma \in \Gamma_{\alpha}$ and in view of (29) and the definition of $\beta_{\alpha}$ we have

$$
\beta_{\alpha} \leq \sup _{s, x_{0}} E_{\alpha}\left(\tilde{\gamma}\left(s, x_{0}\right)\right) \leq \sup _{s, x_{0}} E_{\alpha_{k}}\left(\gamma\left(s, x_{0}\right)\right) \leq \beta_{\alpha}+(B+2)\left(\alpha_{k}-\alpha\right) .
$$

Consider any point $\tilde{u}=\tilde{\gamma}\left(s, x_{0}\right)=i_{k}(u)$, where $u=\gamma\left(s, x_{0}\right)$, satisfying

$$
\beta_{\alpha}-\left(\alpha_{k}-\alpha\right) \leq E_{\alpha}(\tilde{u}) .
$$

For such $u, \tilde{u}$ we then have the chain of inequalities

$$
\begin{aligned}
\beta_{\alpha}-\left(\alpha_{k}-\alpha\right) & \leq E_{\alpha}(\tilde{u}) \leq E_{\alpha_{k}}(\tilde{u}) \leq E_{\alpha_{k}}(u) \\
& \leq \beta_{\alpha}+(B+2)\left(\alpha_{k}-\alpha\right)
\end{aligned}
$$

with interesting consequences.

i) First, observe that (31) implies the estimate

$$
\frac{E_{\alpha_{k}}(\tilde{u})-E_{\alpha}(\tilde{u})}{\alpha_{k}-\alpha} \leq B+3 .
$$


By the mean value theorem and (26) therefore for some number $\alpha^{\prime} \in\left[\alpha, \alpha_{k}\right]$ we obtain

$$
\partial_{\alpha} E_{\alpha^{\prime}}(\tilde{u})=\int_{\Omega} \frac{\left(\alpha^{\prime} \log \left(1+|\nabla \tilde{u}|^{2}\right)-1\right)\left(1+|\nabla \tilde{u}|^{2}\right)^{\alpha^{\prime}}+1}{\left(\alpha^{\prime}\right)^{2}} d x \leq B+3 .
$$

Observing that

$$
\begin{aligned}
& \left(\alpha^{\prime} \log \left(1+s^{2}\right)-1\right)\left(1+s^{2}\right)^{\alpha^{\prime}}-\left(\alpha \log \left(1+s^{2}\right)-1\right)\left(1+s^{2}\right)^{\alpha} \\
& \quad \geq\left(\alpha \log \left(1+s^{2}\right)-1\right)\left(1+s^{2}\right)^{\alpha} \cdot\left[\left(1+s^{2}\right)^{\alpha^{\prime}-\alpha}-1\right] \\
& \quad \geq-C\left(\alpha^{\prime}-\alpha\right)
\end{aligned}
$$

we then can estimate

$$
\begin{aligned}
& \partial_{\alpha} E_{\alpha^{\prime}}(\tilde{u}) \\
& \quad \geq\left(\frac{\alpha}{\alpha^{\prime}}\right)^{2} \int_{\Omega} \frac{\left(\alpha \log \left(1+|\nabla \tilde{u}|^{2}\right)-1\right)\left(1+|\nabla \tilde{u}|^{2}\right)^{\alpha}+1}{\alpha^{2}} d x-C\left(\alpha^{\prime}-\alpha\right) \\
& \quad=\left(\frac{\alpha}{\alpha^{\prime}}\right)^{2} \partial_{\alpha} E_{\alpha}(\tilde{u})-C\left(\alpha^{\prime}-\alpha\right) .
\end{aligned}
$$

Hence for any sequence $\tilde{u}_{k}=i_{k}\left(u_{k}\right), k \in \mathbb{N}$, satisfying (31) for $k \in \mathbb{N}$ we obtain the estimate

$$
\limsup _{k \rightarrow \infty} \partial_{\alpha} E_{\alpha}\left(\tilde{u}_{k}\right) \leq B+3 .
$$

ii) We can also use (31) to compare $d E_{\alpha}(\tilde{u})$ and $d E_{\alpha_{k}}(u)$. We begin by estimating the distance between $u$ and $\tilde{u}=a u$. Using (27), (31), and Lemma 3.4 i) we have

$$
\begin{aligned}
0 & \leq g_{\alpha_{k}}(u)-g_{\alpha}(u) \\
& =\int_{\Omega}\left(\left(1+|\nabla u|^{2}\right)^{\alpha_{k}-1}-\left(1+|\nabla u|^{2}\right)^{\alpha-1}\right)|\nabla u|^{2} d x \\
& \leq \int_{\Omega}\left(\left(1+|\nabla u|^{2}\right)^{\alpha_{k}}-\left(1+|\nabla u|^{2}\right)^{\alpha}\right) d x \\
& \leq \int_{\Omega}\left(\left(1+|\nabla u|^{2}\right)^{\alpha_{k}}-\left(1+|\nabla \tilde{u}|^{2}\right)^{\alpha}\right) d x \\
& =2 \alpha\left(E_{\alpha_{k}}(u)-E_{\alpha}(\tilde{u})\right)+\frac{\alpha_{k}-\alpha}{\alpha_{k}} \int_{\Omega}\left[\left(1+|\nabla u|^{2}\right)^{\alpha_{k}}-1\right] d x \\
& \leq C\left(\alpha_{k}-\alpha\right) .
\end{aligned}
$$

Now observe that for any $a^{\prime} \in[a, 1]$ by Lemma 3.2 there holds

$$
g_{\alpha}\left(a^{\prime} u\right) \leq g_{\alpha}(a u)=0=g_{\alpha_{k}}(u) \leq g_{\alpha_{k}}\left(a^{\prime} u\right) .
$$


Hence for any such number $a^{\prime}$ there is $\alpha^{\prime} \in\left[\alpha, \alpha_{k}\right]$ such that $a^{\prime} u \in M_{\alpha^{\prime}}$. Thus by Lemma 3.2 we can estimate

$$
a^{\prime} \frac{d}{d a^{\prime}} g_{\alpha}\left(a^{\prime} u\right)=\left\langle d g_{\alpha}\left(a^{\prime} u\right), a^{\prime} u\right\rangle \leq\left\langle d g_{\alpha^{\prime}}\left(a^{\prime} u\right), a^{\prime} u\right\rangle \leq-\frac{1}{2} C_{0}
$$

for any $a \leq a^{\prime} \leq 1$. From (33) then it follows that

$$
\begin{aligned}
\frac{1}{2} C_{0}(1-a) & \leq-\int_{a}^{1} \frac{d}{d a^{\prime}} g_{\alpha}\left(a^{\prime} u\right) d a^{\prime}=g_{\alpha}(a u)-g_{\alpha}(u) \\
& =-g_{\alpha}(u)=g_{\alpha_{k}}(u)-g_{\alpha}(u) \leq C\left(\alpha_{k}-\alpha\right)
\end{aligned}
$$

and hence that

$$
1-a \leq C\left(\alpha_{k}-\alpha\right)
$$

Given $v \in W_{0}^{1,2 \alpha_{k}}(\Omega)$, now we can estimate

$$
\begin{aligned}
&\left|\left\langle d E_{\alpha_{k}}(u), v\right\rangle-\left\langle d E_{\alpha}(\tilde{u}), v\right\rangle\right| \\
& \leq\left|\left\langle d E_{\alpha_{k}}(u), v\right\rangle-\left\langle d E_{\alpha}(u), v\right\rangle\right|+\left|\left\langle d E_{\alpha}(u)-d E_{\alpha}(\tilde{u}), v\right\rangle\right| \\
& \leq \int_{\Omega}\left(\left(1+|\nabla u|^{2}\right)^{\alpha_{k}-1}-\left(1+|\nabla u|^{2}\right)^{\alpha-1}\right)|\nabla u||\nabla v| d x \\
& \quad+\int_{\Omega}\left(\left(1+|\nabla u|^{2}\right)^{\alpha-1}-\left(1+a^{2}|\nabla u|^{2}\right)^{\alpha-1} a\right)|\nabla u||\nabla v| d x \\
&+\int_{\Omega}|f(u)-f(a u)||v| d x=I+I I+I I I .
\end{aligned}
$$

Estimating $2|\nabla u||\nabla v| \leq \varepsilon^{-1}|\nabla u|^{2}+\varepsilon|\nabla v|^{2} \leq \varepsilon^{-1}\left(1+|\nabla u|^{2}\right)+\varepsilon|\nabla v|^{2}$ and then using (33) and Young's inequality, the first term can be estimated

$$
\begin{aligned}
I \leq & \varepsilon^{-1} \int_{\Omega}\left(\left(1+|\nabla u|^{2}\right)^{\alpha_{k}}-\left(1+|\nabla u|^{2}\right)^{\alpha}\right) d x \\
& +\varepsilon \int_{\Omega}\left(1+|\nabla u|^{2}\right)^{\alpha_{k}-1}|\nabla v|^{2} d x \\
\leq & C \varepsilon^{-1}\left(\alpha_{k}-\alpha\right)+\varepsilon \frac{\alpha_{k}-1}{\alpha_{k}} \int_{\Omega}\left(1+|\nabla u|^{2}\right)^{\alpha_{k}} d x+\frac{\varepsilon}{\alpha_{k}} \int_{\Omega}|\nabla v|^{2 \alpha_{k}} d x .
\end{aligned}
$$

Choosing $\varepsilon=\sqrt{\alpha_{k}-\alpha}$, we conclude that

$$
I \leq C \sqrt{\alpha_{k}-\alpha} \rightarrow 0(k \rightarrow \infty),
$$

uniformly for all $u$ satisfying (31) and all $v \in W_{0}^{1,2 \alpha_{k}}(\Omega)$ with $\|v\|_{W_{0}^{1,2 \alpha_{k}}(\Omega)} \leq 1$. 
Similarly, we estimate

$$
\begin{aligned}
I I \leq & \varepsilon^{-1} \int_{\Omega}\left(\left(1+|\nabla u|^{2}\right)^{\alpha-1}-\left(1+a^{2}|\nabla u|^{2}\right)^{\alpha-1} a\right)|\nabla u|^{2} d x \\
& +\varepsilon \int_{\Omega}\left(1+|\nabla u|^{2}\right)^{\alpha-1}|\nabla v|^{2} d x \\
\leq & \varepsilon^{-1} \int_{\Omega}\left(\left(1+|\nabla u|^{2}\right)^{\alpha-1}|\nabla u|^{2}-\left(1+a^{2}|\nabla u|^{2}\right)^{\alpha-1} a^{2}|\nabla u|^{2}\right) d x \\
& +\varepsilon \frac{\alpha-1}{\alpha} \int_{\Omega}\left(1+|\nabla u|^{2}\right)^{\alpha}+\frac{\varepsilon}{\alpha} \int_{\Omega}|\nabla v|^{2 \alpha} d x,
\end{aligned}
$$

thereby using the fact that $a \leq 1$ to replace $a$ by $a^{2}$ in the last estimate. Thus, recalling the definition of $g_{\alpha}$ and using that $g_{\alpha}(a u)=0 \geq g_{\alpha}(u)$, we find

$$
\begin{aligned}
I I & \leq \varepsilon^{-1}\left(g_{\alpha}(u)-g_{\alpha}(a u)\right)+\varepsilon^{-1} \int_{\Omega}(f(u) u-f(a u) a u) d x+C \varepsilon \\
& \leq \varepsilon^{-1} \int_{\Omega}(f(u) u-f(a u) a u) d x+C \varepsilon
\end{aligned}
$$

Finally, remark that by Lemma $3.4 \mathrm{i}$ ) the set of $u, \tilde{u}$ satisfying (31) is bounded in $W_{0}^{1,2 \alpha}(\Omega) \hookrightarrow C^{0}(\bar{\Omega})$. By (34) therefore we can uniformly bound

$\|f(u) u-f(a u) a u\|_{L^{\infty}(\Omega)}+\|f(u)-f(a u)\|_{L^{\infty}(\Omega)} \leq C|1-a| \leq C\left(\alpha_{k}-\alpha\right)$.

Consequently, with our choice $\varepsilon=\sqrt{\alpha_{k}-\alpha}$, we obtain

$$
I I+I I I \leq C \varepsilon^{-1}\left(\alpha_{k}-\alpha\right)+C \varepsilon \leq C \sqrt{\alpha_{k}-\alpha},
$$

for all $u$ satisfying (31) and $v$ with $\|v\|_{W_{0}^{1,2 \alpha_{k}(\Omega)}} \leq 1$.

Summarizing, we find that, as $k \rightarrow \infty$,

$$
\sup \left\{\left|\left\langle d E_{\alpha_{k}}(u), v\right\rangle-\left\langle d E_{\alpha}(\tilde{u}), v\right\rangle\right| ;\|v\|_{W_{0}^{1,2 \alpha_{k}(\Omega)}} \leq 1\right\} \rightarrow 0,
$$

uniformly for all $u, \tilde{u}=i_{k}(u)$ as in (31).

iii) Our next aim is to show that there exists a sequence $\left(u_{k}\right)$ satisfying (31) and such that

$$
\left\|d E_{\alpha_{k}}\left(u_{k}\right)\right\|_{T_{u_{k}}^{*} M_{\alpha_{k}}} \rightarrow 0(k \rightarrow \infty) .
$$

Otherwise there exists $\varepsilon>0$ such that

$$
\left\|d E_{\alpha_{k}}(u)\right\|_{T_{u}^{*} M_{\alpha_{k}}} \geq 4 \varepsilon
$$


for all $u$ satisfying (31) and sufficiently large $k$, say $k \geq k_{0}$. For such $k$ then let $e_{k}: M_{\alpha_{k}} \rightarrow W_{0}^{1,2 \alpha_{k}}(\Omega)$ be a locally Lipschitz continuous pseudogradient vector field for $E_{\alpha_{k}}$, satisfying the conditions $e_{k}(u) \in T_{u} M_{\alpha_{k}}$, $\left\|e_{k}(u)\right\|_{W_{0}^{1,2 \alpha_{k}(\Omega)}}<1$, and

$$
\left\langle d E_{\alpha_{k}}(u), e_{k}(u)\right\rangle<-\frac{1}{2}\left\|E_{\alpha_{k}}(u)\right\|_{T_{u}^{*} M_{\alpha_{k}}} \leq-2 \varepsilon
$$

for all $u \in M_{\alpha_{k}}$ satisfying (31).

Let $\psi \in C^{\infty}(\mathbb{R})$ be a cut-off function such that $0 \leq \psi \leq 1, \psi(s)=0$ for $s \leq 0, \psi(s)=1$ for $s \geq 1$, and for $k \geq k_{0}$ let

$$
\psi_{k}(u)=\psi\left(\frac{E_{\alpha}\left(i_{k}(u)\right)-\left(\beta_{\alpha}-\left(\alpha_{k}-\alpha\right)\right)}{\alpha_{k}-\alpha}\right), u \in M_{\alpha_{k}} .
$$

Recalling that $i_{k}: M_{\alpha_{k}} \rightarrow M_{\alpha}$ is Lipschitz, the truncated vector field $\tilde{e}_{k}$, given by

$$
\tilde{e}_{k}(u)=\psi_{k}(u) e_{k}(u)
$$

then defines a Lipschitz continuous tangent vector field on $M_{\alpha_{k}}$. Let $\Phi_{k}$ : $\left[0, \infty\left[\times M_{\alpha_{k}} \rightarrow M_{\alpha_{k}}\right.\right.$ be the truncated pseudo gradient-flow generated by $\tilde{e}_{k}$, satisfying

$$
\frac{d}{d t} \Phi_{k}(t, u)=\tilde{e}_{k}\left(\Phi_{k}(t, u)\right), t>0
$$

with initial data $\Phi_{k}(0, u)=u$ for all $u$.

Note that

$$
\begin{aligned}
\frac{d}{d t} E_{\alpha_{k}}\left(\Phi_{k}(t, u)\right)_{\mid t=0} & =\left\langle d E_{\alpha_{k}}(u), \frac{d}{d t} \Phi_{k}(t, u)_{\mid t=0}\right\rangle \\
& =\psi_{k}(u)\left\langle d E_{\alpha_{k}}(u), e_{k}(u)\right\rangle \leq 0
\end{aligned}
$$

for all $u \in M_{\alpha_{k}}$.

Moreover, for sufficiently large $k \geq k_{0}$, by Lemma 3.11 there holds

$$
\sup _{x_{0}} E_{\alpha}\left(w_{s_{\delta}, x_{0}, \alpha}\right)<1 / 2+\delta<\beta_{\alpha}-\left(\alpha_{k}-\alpha\right) .
$$

Hence $\psi_{k}\left(w_{s_{\delta}, x_{0}, \alpha_{k}}\right)=0$ and $\Phi_{k}(t, \cdot)$ fixes $w_{s_{\delta}, x_{0}, \alpha_{k}}$ for large $k$ and any $t \geq 0$.

Let $\tilde{\Phi}_{k}=i_{k} \circ \Phi_{k} \circ i_{k}^{-1}:\left[0, \infty\left[\times M_{\alpha} \rightarrow M_{\alpha}\right.\right.$ be the induced flow on $M_{\alpha}$. We claim that also $t \mapsto E_{\alpha}\left(\tilde{\Phi}_{k}(t, u)\right)$ is non-increasing near $t=0$ for any $u \in M_{\alpha_{k}}$ satisfying (31), $k \geq k_{0}$. Write $\tilde{\Phi}_{k}(t, u)=a \Phi_{k}(t, u)$, where 
$a=a\left(\Phi_{k}(t, u)\right)$. By (35) then, with error $o(1) \rightarrow 0$ as $k \rightarrow \infty$, we obtain

$$
\begin{aligned}
\frac{d}{d t} E_{\alpha}\left(\tilde{\Phi}_{k}(t, u)\right)_{\left.\right|_{t=0}} & =\left\langle d E_{\alpha}(\tilde{u}), \frac{d}{d t} \tilde{\Phi}_{k}(t, u)_{\mid t=0}\right\rangle \\
& =a\left\langle d E_{\alpha}(\tilde{u}), \frac{d}{d t} \Phi_{k}(t, u)_{\left.\right|_{t=0}}\right\rangle \\
& =a\left\langle d E_{\alpha}(\tilde{u}), \tilde{e}_{k}(u)\right\rangle=a \psi_{k}(u)\left\langle d E_{\alpha}(\tilde{u}), e_{k}(u)\right\rangle \\
& =a \psi_{k}(u)\left(\left\langle d E_{\alpha_{k}}(u), e_{k}(u)\right\rangle+o(1)\right) \\
& \leq-2 \psi_{k}(u) \varepsilon+o(1) .
\end{aligned}
$$

Here we also used differentiability of the map $u \mapsto a=a(u)$ and the fact that

$$
\left\langle d E_{\alpha}(\tilde{u}), \frac{d}{d t}\left(a\left(\Phi_{k}(t, u)\right)\right)_{\left.\right|_{t=0}} \cdot u\right\rangle=a^{-1} \frac{d}{d t}\left(a\left(\Phi_{k}(t, u)\right)\right)_{\left.\right|_{t=0}}\left\langle d E_{\alpha}(\tilde{u}), \tilde{u}\right\rangle=0 .
$$

For $\gamma \in \Gamma_{\alpha_{k}}$ as above with corresponding $\tilde{\gamma}=i_{k} \circ \gamma \in \Gamma_{\alpha}$ define the 1-parameter family of maps $\gamma_{t}=\Phi_{k}(t, \cdot) \circ \gamma \in \Gamma_{\alpha_{k}}$ and let $\tilde{\gamma}_{t}=i_{k} \circ \gamma_{t} \in \Gamma_{\alpha}$. Then for $t \geq 0$ we have

$$
\sup _{s, x_{0}} E_{\alpha_{k}}\left(\gamma_{t}\left(s, x_{0}\right)\right) \leq \sup _{s, x_{0}} E_{\alpha_{k}}\left(\gamma\left(s, x_{0}\right)\right) \leq \beta_{\alpha_{k}}+\left(\alpha_{k}-\alpha\right) .
$$

Hence

$$
M(t):=\sup _{s, x_{0}} E_{\alpha}\left(\tilde{\gamma}_{t}\left(s, x_{0}\right)\right) \geq \beta_{\alpha}
$$

is attained only at points $\tilde{u}=i_{k}(u)$ satisfying (31). Note that $\psi_{k}(u)=1$ at such points. From (37) for $k \geq k_{0}$ it then follows that

$$
\frac{d}{d t} M(t) \leq-\varepsilon<0
$$

and therefore $M(t)<\beta_{\alpha}$ for sufficiently large $t$, contradicting the definition of $\beta_{\alpha}$. Hence (36) must hold true.

iv) We can now complete the proof as follows.

Let $\left(u_{k}\right)$ be a sequence satisfying (31) and (36). By Lemma 3.4. i) then we have $\left\|u_{k}\right\|_{W_{0}^{1,2 \alpha_{k}(\Omega)}} \leq C$ uniformly in $k \in \mathbb{N}$ and Lemma 3.3 gives

$$
\left\|d E_{\alpha_{k}}\left(u_{k}\right)\right\|_{W^{-1,2 \alpha_{k}(\Omega)}} \rightarrow 0(k \rightarrow \infty) .
$$

Since, in particular, $\left(u_{k}\right)$ is bounded in $W_{0}^{1,2 \alpha}(\Omega)$, we may assume that $u_{k} \rightarrow u$ weakly in $W_{0}^{1,2 \alpha}(\Omega)$ and uniformly on $\bar{\Omega}$ as $k \rightarrow \infty$. Moreover, by density of $C_{0}^{\infty}(\Omega)$ in $W_{0}^{1,2 \alpha}(\Omega)$, we can find a sequence of smooth functions 
$\left(u^{l}\right)$ such that $u^{l} \rightarrow u$ strongly in $W_{0}^{1,2 \alpha}(\Omega)$ as $l \rightarrow \infty$. For any fixed $l \in \mathbb{N}$, then with error $o(1) \rightarrow 0$ as $k \rightarrow \infty$ from (20) and (38) we derive

$$
\begin{aligned}
o(1)= & \left\langle d E_{\alpha_{k}}\left(u_{k}\right), u_{k}-u^{l}\right\rangle \\
\geq & \int_{\Omega}\left(\frac{\left(1+\left|\nabla u_{k}\right|^{2}\right)^{\alpha_{k}}-\left(1+\left|\nabla u^{l}\right|^{2}\right)^{\alpha_{k}}}{2 \alpha_{k}}+\frac{\left|\nabla\left(u_{k}-u^{l}\right)\right|^{2}}{2}\right) d x \\
& -\int_{\Omega} f\left(u_{k}\right)\left(u_{k}-u^{l}\right) d x \\
\geq & \int_{\Omega}\left(\frac{\left(1+\left|\nabla u_{k}\right|^{2}\right)^{\alpha}-\left(1+\left|\nabla u^{l}\right|^{2}\right)^{\alpha}}{2 \alpha}+\frac{\left|\nabla\left(u_{k}-u^{l}\right)\right|^{2}}{2}\right) d x \\
& -\int_{\Omega} f(u)\left(u-u^{l}\right) d x+o(1) .
\end{aligned}
$$

Also letting $l \rightarrow \infty$, we conclude that $u_{k} \rightarrow u$ strongly in $W_{0}^{1,2 \alpha}(\Omega)$ and $E_{\alpha_{k}}\left(u_{k}\right) \rightarrow E_{\alpha}(u)$ as $k \rightarrow \infty$. For any $w \in C_{0}^{\infty}(\Omega)$ then

$$
\left\langle d E_{\alpha}(u), w\right\rangle=\lim _{k \rightarrow \infty}\left\langle d E_{\alpha_{k}}\left(u_{k}\right), w\right\rangle=0,
$$

and $u \in M_{\alpha}$ is a critical point of $E_{\alpha}$ with $E_{\alpha}(u)=\beta_{\alpha}$. Moreover, $\tilde{u}_{k}=a\left(u_{k}\right) u_{k} \rightarrow u(k \rightarrow \infty)$ in view of (34). Finally, the function $s \mapsto$ $\alpha \log \left(1+s^{2}\right)\left(1+s^{2}\right)^{\alpha}$ being convex, the functional $\partial_{\alpha} E_{\alpha}$ given by (26) is lower semi-continuous in $W_{0}^{1,2 \alpha}(\Omega)$. Hence, from (32) we conclude that

$$
\partial_{\alpha} E_{\alpha}(u) \leq \liminf _{k \rightarrow \infty} \partial_{\alpha} E_{\alpha}\left(\tilde{u}_{k}\right) \leq B+3 .
$$

The proof is complete.

Theorem 1.2 is an immediate consequence of Lemma 3.11, Lemma 3.12, Lemma 3.13, and (30).

\section{Convergence}

In this section we give the proof of Theorem 1.3. For a suitable sequence of numbers $\alpha \searrow 1$ let $u_{\alpha} \in W_{0}^{1,2 \alpha}(\Omega)$ be solutions to equation (11) with energy $\left.E_{\alpha}\left(u_{\alpha}\right)=\beta_{\alpha} \rightarrow \beta \in\right] \frac{1}{2}, 1[$ as $\alpha \rightarrow 1$ and satisfying condition (12), which in view of (26) and the uniform bound

$$
\left\|u_{\alpha}\right\|_{W_{0}^{1,2 \alpha}(\Omega)}^{2 \alpha} \leq 4 \alpha E_{\alpha}\left(u_{\alpha}\right)+C \leq C
$$

from Lemma 3.4 i) we may rephrase as

$$
\liminf _{\alpha \rightarrow 1}\left((\alpha-1) \log \left(\frac{1}{\alpha-1}\right) \int_{\Omega} \log \left(1+\left|\nabla u_{\alpha}\right|^{2}\right)\left(1+\left|\nabla u_{\alpha}\right|^{2}\right)^{\alpha} d x\right)=0 .
$$


By Lemma 3.5 and assumption (21) we may assume that, as $\alpha \rightarrow 1$, $u_{\alpha} \rightarrow 0$ weakly in $H_{0}^{1}(\Omega)$ and that

$$
\left|\nabla u_{\alpha}\right|^{2 \alpha} d x \rightarrow 2 \beta \delta_{x_{0}}, u_{\alpha} f\left(u_{\alpha}\right) d x \rightarrow 2 \beta \delta_{x_{0}}
$$

weakly in the sense of measures. Otherwise, as $\alpha \rightarrow 1$ a sub-sequence $u_{\alpha} \rightarrow u$ strongly in $H_{0}^{1}(\Omega)$ where $u$ solves (1) with energy $E(u)=\beta$, and the proof of Theorem 1.3 is complete.

Clearly, we may pass to further sub-sequences, still denoted as $\left(u_{\alpha}\right)$, if necessary.

\subsection{Blow-up analysis}

We rescale as in [5]. For a suitable number $0<\varepsilon<1$ determined in Lemmas 4.4, 4.7, and 4.8 below, we (tentatively) choose $r_{\alpha}>0, x_{\alpha} \in \Omega$ such that

$$
\frac{\varepsilon}{2} \leq \int_{B_{r_{\alpha}}\left(x_{\alpha}\right)} f\left(u_{\alpha}\right) u_{\alpha} d x=\sup _{x_{0} \in \Omega} \int_{B_{r_{\alpha}}\left(x_{0}\right)} f\left(u_{\alpha}\right) u_{\alpha} d x \leq \varepsilon .
$$

Observe that (41) implies that $r_{\alpha} \rightarrow 0$ as $\alpha \rightarrow 1$. Moreover, we also obtain some preliminary estimate on the rate of convergence.

Lemma 4.1. $\lim \sup _{\alpha \rightarrow 1} r_{\alpha}^{1-\alpha}<\infty$.

Proof. In view of (9) and Theorem 2.4 we have

$$
\begin{aligned}
\frac{\varepsilon}{2} & \leq \int_{B_{r_{\alpha}}\left(x_{\alpha}\right)} f\left(u_{\alpha}\right) u_{\alpha} d x \leq \pi r_{\alpha}^{2} \cdot \sup _{\Omega} u_{\alpha}^{2} e^{\varphi\left(u_{\alpha}\right)} \\
& \leq \pi \exp \left(\sup _{\Omega}\left(4 \pi u_{\alpha}^{2}+2 \log \left(u_{\alpha} r_{\alpha}\right)\right)\right) \leq \pi \exp \left(\frac{C}{\alpha-1}+2 \log r_{\alpha}\right) \\
& =\pi \exp \left(\frac{2}{\alpha-1}\left(C-\log r_{\alpha}^{1-\alpha}\right)\right) .
\end{aligned}
$$

The claim follows.

Scale

$$
\Omega_{\alpha}=\left\{x \in \mathbb{R}^{2} ; x_{\alpha}+r_{\alpha} x \in \Omega\right\},
$$

and let

$$
v_{\alpha}(x)=u_{\alpha}\left(x_{\alpha}+r_{\alpha} x\right) \in H_{0}^{1}\left(\Omega_{\alpha}\right),
$$

satisfying the equation

$$
-\operatorname{div}\left[\left(1+r_{\alpha}^{-2}\left|\nabla v_{\alpha}\right|^{2}\right)^{\alpha-1} \nabla v_{\alpha}\right]=r_{\alpha}^{2} f\left(v_{\alpha}\right) \text { in } \Omega_{\alpha}
$$


and the normalization condition

$$
\frac{\varepsilon}{2} \leq \int_{B_{1}(0)} r_{\alpha}^{2} f\left(v_{\alpha}\right) v_{\alpha} d x=\sup _{x_{0} \in \Omega_{\alpha}} \int_{B_{1}\left(x_{0}\right)} r_{\alpha}^{2} f\left(v_{\alpha}\right) v_{\alpha} d x \leq \varepsilon .
$$

In non-divergence form, equation (42) may be written as

$$
L_{\alpha} v_{\alpha}=\Delta v_{\alpha}+2(\alpha-1) \frac{\partial_{i} v_{\alpha} \partial_{i j} v_{\alpha} \partial_{j} v_{\alpha}}{r_{\alpha}^{2}+\left|\nabla v_{\alpha}\right|^{2}}=-\frac{1}{\left(1+r_{\alpha}^{-2}\left|\nabla v_{\alpha}\right|^{2}\right)^{\alpha-1}} r_{\alpha}^{2} f\left(v_{\alpha}\right)
$$

with a uniformly elliptic operator

$$
L_{\alpha} w=a_{\alpha}^{i j}(x) \partial_{i j} w
$$

with coefficients $a_{\alpha}^{i j}=\delta^{i j}+A_{\alpha}^{i j}$ satisfying

$$
A_{\alpha}^{i j}=2(\alpha-1) \frac{\partial_{i} v_{\alpha} \partial_{j} v_{\alpha}}{r_{\alpha}^{2}+\left|\nabla v_{\alpha}\right|^{2}} \rightarrow 0
$$

uniformly as $\alpha \rightarrow 1$.

We extend $v_{\alpha}$ as $v_{\alpha} \equiv 0$ on $\mathbb{R}^{2} \backslash \Omega_{\alpha}$. Passing to a sub-sequence $\alpha \rightarrow 1$, we may also assume that $\Omega_{\alpha} \rightarrow \Omega_{1}$, where $\Omega_{1}$ is the plane $\mathbb{R}^{2}$ or a half-space.

For $y \in \mathbb{R}^{2}, r>0$ decompose

$$
v_{\alpha}=w_{\alpha}+c_{\alpha},
$$

where $c_{\alpha}$ denotes the mean value

$$
c_{\alpha}=c_{\alpha}(y, r)=f_{B_{r}(y)} v_{\alpha}(x) d x .
$$

Observe that (41) implies the uniform estimate

$$
\int_{B_{r}(y)}\left|\nabla w_{\alpha}\right|^{2} d x=\int_{B_{r}(y)}\left|\nabla v_{\alpha}\right|^{2} d x \leq \int_{\Omega}\left|\nabla u_{\alpha}\right|^{2} d x \leq 2
$$

for small $\alpha>1$.

Moreover, as in [5], Lemma 3.3, there holds

Lemma 4.2. For any $y \in \mathbb{R}^{2}$, any $r>0$ we have $w_{\alpha} \rightarrow 0$ weakly in $H^{1}\left(B_{r}(y)\right)$. If $r<1$, in addition we obtain

$$
\limsup _{\alpha \rightarrow 1} \int_{B_{r}(y)}\left|\nabla w_{\alpha}\right|^{2} d x \leq \varepsilon .
$$


Proof. Fix $y \in \mathbb{R}^{2}, r>0$, and $c_{\alpha}=c_{\alpha}(y, r)$. First consider the case $\Omega_{1}=\mathbb{R}^{2}$. For any $R>0$ consider the function $\tilde{v}_{\alpha}=v_{\alpha}-c_{\alpha} \in H^{1}\left(B_{R}(y)\right)$. Since the mean value of $\tilde{v}_{\alpha}$ on $B_{r}(y)$ vanishes, by Poincarés inquality and (47) the family $\left(\tilde{v}_{\alpha}\right)_{\alpha>1}$ is bounded in $H^{1}\left(B_{R}(y)\right)$. Hence we may extract a weakly convergent subsequence $\tilde{v}_{\alpha} \rightarrow \tilde{v}$ as $\alpha \rightarrow 1$ where $\tilde{v}$ is harmonic. Indeed, for any $\psi \in C_{0}^{\infty}\left(B_{R}(y)\right)$ we have

$$
\begin{aligned}
\int_{B_{R}(y)}\left(1+r_{\alpha}^{-2}\left|\nabla v_{\alpha}\right|^{2}\right)^{\alpha-1} & \nabla \tilde{v}_{\alpha} \nabla \psi d x \\
& =r_{\alpha}^{2} \int_{B_{R}(y)} f\left(v_{\alpha}\right) \psi d x \leq C \int_{\Omega}\left|f\left(u_{\alpha}\right)\right| d x \rightarrow 0 .
\end{aligned}
$$

Using Lemma 4.1 and the fact that $\left(1+\left|\nabla v_{\alpha}\right|^{2}\right)^{\alpha-1} \rightarrow 1$ in $L_{\text {loc }}^{2}\left(\mathbb{R}^{2}\right)$ as $\alpha \rightarrow 1$ we can also pass to the limit $\alpha \rightarrow 1$ on the left to conclude that $\int_{B_{R}(y)} \nabla \tilde{v} \nabla \psi d x=0$ for all such $\psi$.

Choosing $R=R(\alpha) \rightarrow \infty$ suitably, we may assume that $\tilde{v}_{\alpha} \rightarrow \tilde{v}$ weakly locally in $H^{1}$, where $\tilde{v} \in H_{\mathrm{loc}}^{1}\left(\mathbb{R}^{2}\right)$ is harmonic with

$$
\int_{\mathbb{R}^{2}}|\nabla \tilde{v}|^{2} d x \leq \liminf _{\alpha \rightarrow 1} \int_{B_{R \alpha}(y)}\left|\nabla \tilde{v}_{\alpha}\right|^{2} d x \leq 2
$$

and $f_{B_{r}(y)} \tilde{v} d x=0$.

It follows that $\tilde{v} \equiv 0$. Since $w_{\alpha}=\tilde{v}_{\alpha \mid B_{r}(y)}$, we conclude that $w_{\alpha} \rightarrow 0$ weakly in $H^{1}\left(B_{r}(y)\right)$ as $\alpha \rightarrow 1$.

To obtain the second assertion, consider the decomposition (46) on $B_{1}(y)$. Fix any cut-off function $\psi \in C_{0}^{\infty}\left(B_{1}(y)\right)$ satisfying $0 \leq \psi \leq 1$. Upon testing equation (42) with the function $\psi w_{\alpha}$, in view of Lemma 4.1 then with a uniform constant $C$ depending on $\psi$ we obtain

$$
\begin{aligned}
\int_{\mathbb{R}^{2}} & \left.\nabla w_{\alpha}\right|^{2} \psi d x \leq \int_{\mathbb{R}^{2}}\left(1+r_{\alpha}^{-2}\left|\nabla v_{\alpha}\right|^{2}\right)^{\alpha-1}\left|\nabla w_{\alpha}\right|^{2} \psi d x \\
& =\int_{\mathbb{R}^{2}} r_{\alpha}^{2} f\left(v_{\alpha}\right) w_{\alpha} \psi d x-\int_{\mathbb{R}^{2}}\left(1+r_{\alpha}^{-2}\left|\nabla v_{\alpha}\right|^{2}\right)^{\alpha-1} \nabla v_{\alpha} \nabla \psi w_{\alpha} d x \\
& \leq \int_{\mathbb{R}^{2}} r_{\alpha}^{2} f\left(v_{\alpha}\right) v_{\alpha} \psi d x+C \int_{B_{1}(y)}\left(1+r_{\alpha}^{-2}\left|\nabla v_{\alpha}\right|^{2}\right)^{\alpha-1}\left|\nabla v_{\alpha}\right|\left|w_{\alpha}\right| d x \\
& \leq \varepsilon+C \int_{B_{1}(y)}\left(\left|\nabla v_{\alpha}\right|+\left|\nabla v_{\alpha}\right|^{2 \alpha-1}\right)\left|w_{\alpha}\right| d x .
\end{aligned}
$$

By weak convergence $w_{\alpha} \rightarrow 0$ in $H^{1}\left(B_{1}(y)\right)$ and Rellich's compactness theorem we also have $w_{\alpha} \rightarrow 0$ in $L^{p}\left(B_{1}(y)\right)$ for any $p<\infty$. Hence, as $\alpha \rightarrow 1$ we find

$$
\limsup _{\alpha \rightarrow 1} \int_{\mathbb{R}^{2}}\left|\nabla w_{\alpha}\right|^{2} \psi d x \leq \varepsilon
$$


for any $\psi$ as above. Given $r<1$, we can find $\psi \in C_{0}^{\infty}\left(B_{1}(y)\right)$ such that $0 \leq \psi \leq 1$ and $\psi=1$ on $B_{r}(y)$. Since $\nabla w_{\alpha}=\nabla v_{\alpha}$ is independent of the domain $B_{r}(y)$ of decomposition (46), this proves our claim in case $\Omega_{1}=\mathbb{R}^{2}$.

If $\Omega_{1}$ is a half-space we consider $v_{\alpha}$ instead of $\tilde{v}_{\alpha}$. From (42) and (47) then we deduce that $\left(v_{\alpha}\right)$ weakly accumulates in $H_{\mathrm{loc}}^{1}\left(\mathbb{R}^{2}\right)$ at a function $v$ satisfying the conditions $\Delta v=0$ in $\Omega_{1}, v=0$ on $\partial \Omega_{1}$, and $\nabla v \in L^{2}\left(\mathbb{R}^{2}\right)$. Again it follows that $v \equiv 0$, proving that $v_{\alpha} \rightarrow 0$ weakly in $H^{1}\left(B_{r}(y)\right)$ as $\alpha \rightarrow 1$ for any $r>0, y \in \mathbb{R}^{2}$. Hence also $w_{\alpha} \rightarrow$ const. weakly in $H^{1}\left(B_{r}(y)\right)$, and, in fact, $w_{\alpha} \rightarrow 0$ since the mean of $w_{\alpha}$ vanishes.

The second assertion follows as above for the case $\Omega_{1}=\mathbb{R}^{2}$, using the function $\psi v_{\alpha}$ as testing function in (42).

Lemma 4.3. For $y_{1}, y_{2} \in \mathbb{R}^{2}$ and $r_{1}, r_{2}>0$, letting $\left|y_{1}-y_{2}\right|+r_{1}+r_{2}=2 r$, there holds

$$
\left|c_{\alpha}\left(y_{1}, r_{1}\right)-c_{\alpha}\left(y_{2}, r_{2}\right)\right| \leq C+2 \log \left(\frac{r^{2}}{r_{1} r_{2}}\right)
$$

with an absolute constant $C$.

Proof. Choose the point $y$ on the segment connecting $y_{1}$ and $y_{2}$ such that $B_{r_{1}}\left(y_{1}\right) \cup B_{r_{2}}\left(y_{2}\right) \subset B_{r}(y)$, and let $v_{\alpha}=w_{\alpha}+c_{\alpha}$ be the decomposition of $v_{\alpha}$ on $B_{r}(y)$. Also decompose $v_{\alpha}=w_{\alpha}^{i}+c_{\alpha}^{i}$ on $B_{r_{i}}\left(y_{i}\right) \subset B_{r}(y), i=1,2$.

Then, by Jensen's inequality, for each $i=1,2$ we obtain

$$
\begin{aligned}
\left|c_{\alpha}^{i}-c_{\alpha}\right| & =\left|f_{B_{r_{i}}\left(y_{i}\right)}\left(w_{\alpha}-w_{\alpha}^{i}\right) d x\right|=\left|f_{B_{r_{i}}\left(y_{i}\right)} w_{\alpha} d x\right| \\
& \leq f_{B_{r_{i}}\left(y_{i}\right)}\left|w_{\alpha}\right| d x \leq \log \left(f_{B_{r_{i}}\left(y_{i}\right)} e^{\left|w_{\alpha}\right|} d x\right) \\
& \leq 2 \log \left(\frac{r}{r_{i}}\right)+\log \left(f_{B_{r}(y)} e^{\left|w_{\alpha}\right|} d x\right) .
\end{aligned}
$$

Estimating

$$
\left|w_{\alpha}\right| \leq 2 \pi w_{\alpha}^{2} /\left\|\nabla w_{\alpha}\right\|_{L^{2}\left(B_{r}(y)\right)}^{2}+\frac{1}{8 \pi}\left\|\nabla w_{\alpha}\right\|_{L^{2}\left(B_{r}(y)\right)}^{2},
$$

in view of Theorem 2.1 and (47) we have

$$
\log \left(f_{B_{r}(y)} e^{\left|w_{\alpha}\right|} d x\right) \leq C
$$

uniformly for all $\alpha, y$, and $r$, and thus

$$
\left|c_{\alpha}^{i}-c_{\alpha}\right| \leq C+2 \log \left(\frac{r}{r_{i}}\right), i=1,2 .
$$

The claim follows. 
Lemma 4.4. Suppose $\sup _{\alpha}\left|c_{\alpha}\right|<\infty$, where $c_{\alpha}=c_{\alpha}(y, r)$ for some $y \in \Omega_{\alpha}$ and some $0<r<1$. Then a subsequence $v_{\alpha} \rightarrow v$ in $H_{\mathrm{loc}}^{1}\left(B_{r}(y)\right)$.

Proof. By uniform boundedness of $\left(w_{\alpha}\right)$ in $H^{1}\left(B_{r}(y)\right)$, boundedness of $\left(c_{\alpha}\right)$ implies that $\left(v_{\alpha}\right)$ is bounded in $H^{1}\left(B_{r}(y)\right)$. Hence we may assume that $v_{\alpha} \rightarrow v_{0}$ weakly in $H^{1}\left(B_{r}(y)\right)$ as $\alpha \rightarrow 1$, and strongly in $L^{2}\left(B_{r}(y)\right)$.

Moreover, recalling that $\int_{B_{r}(y)} w_{\alpha} d x=0$ and using Lemma 4.2, for $\varepsilon \leq \varepsilon_{1}=\frac{1}{5}$ from Theorem 2.1 we infer that

$$
\int_{B_{r}(y)} e^{10 \pi w_{\alpha}^{2}} d x \leq \int_{B_{r}(y)} e^{2 \pi w_{\alpha}^{2} /\left\|\nabla w_{\alpha}\right\|_{L^{2}\left(B_{r}(y)\right)}^{2}} d x \leq C .
$$

Hence, estimating $\left|v_{\alpha}\right|^{2}=\left|w_{\alpha}+c_{\alpha}\right|^{2} \leq \frac{9}{8}\left|w_{\alpha}\right|^{2}+C$, we find

$$
\begin{aligned}
\int_{B_{r}(y)} & \left.f\left(v_{\alpha}\right)\right|^{2} d x \leq C \int_{B_{r}(y)}\left(1+\left|v_{\alpha}\right|^{2}\right) e^{8 \pi v_{\alpha}^{2}} d x \\
& \leq C \int_{B_{r}(y)}\left(1+\left|w_{\alpha}\right|^{2}\right) e^{9 \pi w_{\alpha}^{2}} d x \leq C \int_{B_{r}(y)} e^{10 \pi w_{\alpha}^{2}} d x \leq C,
\end{aligned}
$$

uniformly in $\alpha$.

Localizing (44) with arbitrary $\psi \in C_{0}^{\infty}\left(B_{r}(y)\right)$, then we find that

$$
L_{\alpha}\left(v_{\alpha} \psi\right)=\psi L_{\alpha} v_{\alpha}+2 a_{\alpha}^{i j} \partial_{i} v_{\alpha} \partial_{j} \psi+v_{\alpha} L_{\alpha} \psi
$$

is bounded in $L^{2}\left(\Omega_{\alpha} \cap B_{r}(y)\right)$ as $\alpha \rightarrow 1$. Writing

$$
-\Delta\left(v_{\alpha} \psi\right)=A_{\alpha}^{i j} \partial_{i j}\left(v_{\alpha} \psi\right)-L_{\alpha}\left(v_{\alpha} \psi\right),
$$

from the Calderòn-Zygmund estimate for $-\Delta$ on $H^{2} \cap H_{0}^{1}\left(\Omega_{\alpha} \cap B_{r}(y)\right)$ and (45) we obtain

$$
\begin{aligned}
\left\|v_{\alpha} \psi\right\|_{H^{2}\left(\Omega_{\alpha} \cap B_{r}(y)\right)} & \leq C\left\|\Delta\left(v_{\alpha} \psi\right)\right\|_{L^{2}\left(\Omega_{\alpha} \cap B_{r}(y)\right)} \\
& \leq C(\alpha-1)\left\|v_{\alpha} \psi\right\|_{H^{2}\left(\Omega_{\alpha} \cap B_{r}(y)\right)}+C .
\end{aligned}
$$

Hence $v_{\alpha}$ is bounded in $H_{\mathrm{loc}}^{2}\left(\bar{\Omega}_{\alpha} \cap B_{r}(y)\right)$ and therefore, by the RellichKondrakov theorem, $v_{\alpha} \rightarrow v_{0}$ strongly in $H_{\mathrm{loc}}^{1}\left(B_{r}(y)\right)$ as $\alpha \rightarrow 1$.

Lemma 4.5. For any $y \in \mathbb{R}^{2}$, any $r>0$ there holds $c_{\alpha}(y, r) \rightarrow \infty$ as $\alpha \rightarrow \infty$. In particular, the sequence $\left(\Omega_{\alpha}\right)$ exhausts $\mathbb{R}^{2}$.

Proof. Let

$$
A=\left\{y \in \mathbb{R}^{2} ; \liminf _{\alpha \rightarrow 1}\left|c_{\alpha}(y, r)\right|<\infty \text { for some } r>0\right\} .
$$

By (48), either $A=\emptyset$, or $A=\mathbb{R}^{2}$; moreover, $y \in A$ if and only if $\left(c_{\alpha}(y, 1)\right)$ is bounded. Also observe that in the case that the sequence $\left(\Omega_{\alpha}\right)$ 
only exhausts a half-space $\mathbb{R}_{+}^{2}$ any point $y \notin \overline{\mathbb{R}_{+}^{2}}$ satisfies $c_{\alpha}(y, r)=0$ for $r<\operatorname{dist}\left(y, \mathbb{R}_{+}^{2}\right)$ and sufficiently small $\alpha>1$. Hence in this case necessarily $A=\mathbb{R}^{2}$.

We now show that $A=\emptyset$ is the only possibility compatible with the normalization (43). In particular then, the sequence $\left(\Omega_{\alpha}\right)$ will exhaust all of $\mathbb{R}^{2}$.

Indeed, suppose by contradiction that $A=\mathbb{R}^{2}$. Then by Lemma 4.4, applied on a cover of $\mathbb{R}^{2}$ by balls of radius $1 / 2$, a subsequence $v_{\alpha} \rightarrow v$ in $H_{\mathrm{loc}}^{1}\left(\mathbb{R}^{2}\right)$, and

$$
\left.\int_{B_{1}(0)} r_{\alpha}^{2} f\left(v_{\alpha}\right) v_{\alpha}\right) d x \rightarrow 0
$$

contradicting (43).

Thus, $A=\emptyset$ and $c_{\alpha}(y, r) \rightarrow \infty$ as $\alpha \rightarrow 1$ for any $y \in \mathbb{R}^{2}$, any $r>0$.

Fix $y \in \mathbb{R}^{2}, r>0$, and decompose

$$
v_{\alpha}=w_{\alpha}+c_{\alpha}=c_{\alpha}\left(1+\frac{w_{\alpha}}{c_{\alpha}}\right) \text { on } B_{r}(y)
$$

as above. Express

$$
\begin{aligned}
r_{\alpha}^{2} f\left(v_{\alpha}\right) v_{\alpha} & =r_{\alpha}^{2} v_{\alpha}^{2} e^{\varphi\left(v_{\alpha}\right)}=\exp \left(\varphi\left(v_{\alpha}\right)+2 \log v_{\alpha}+2 \log r_{\alpha}\right) \\
& =\exp \left(\varphi\left(v_{\alpha}\right)+2 \log \left(c_{\alpha} r_{\alpha}\right)+2 \log \left(1+\frac{w_{\alpha}}{c_{\alpha}}\right)\right)
\end{aligned}
$$

and let

$$
\varphi\left(v_{\alpha}\right)=\varphi\left(c_{\alpha}\right)+\varphi^{\prime}\left(c_{\alpha}\right) w_{\alpha}+R\left(c_{\alpha}, w_{\alpha}\right) .
$$

On account of (9) and since $v_{\alpha} \geq 0$ we can bound the remainder

$$
0 \leq R\left(c_{\alpha}, w_{\alpha}\right) \leq 4 \pi w_{\alpha}^{2} .
$$

Define

$$
\eta_{\alpha}=\varphi\left(c_{\alpha}\right)+\varphi^{\prime}\left(c_{\alpha}\right) w_{\alpha}+2 \log \left(c_{\alpha} r_{\alpha}\right)
$$

so that

$$
r_{\alpha}^{2} f\left(v_{\alpha}\right) v_{\alpha}=W_{\alpha} e^{\eta_{\alpha}}
$$

where

$$
W_{\alpha}=\exp \left(R\left(c_{\alpha}, w_{\alpha}\right)+2 \log \left(1+\frac{w_{\alpha}}{c_{\alpha}}\right)\right) \geq\left(1+\frac{w_{\alpha}}{c_{\alpha}}\right)^{2} .
$$


Observe that $\eta_{\alpha}$ satisfies the equation

$$
\begin{aligned}
& -\operatorname{div}\left[\left(1+R_{\alpha}^{-2}\left|\nabla \eta_{\alpha}\right|^{2}\right)^{\alpha-1} \nabla \eta_{\alpha}\right] \\
& \quad=-\varphi^{\prime}\left(c_{\alpha}\right) \operatorname{div}\left[\left(1+r_{\alpha}^{-2}\left|\nabla v_{\alpha}\right|^{2}\right)^{\alpha-1} \nabla v_{\alpha}\right]=\varphi^{\prime}\left(c_{\alpha}\right) r_{\alpha}^{2} f\left(v_{\alpha}\right),
\end{aligned}
$$

where $R_{\alpha}=\varphi^{\prime}\left(c_{\alpha}\right) r_{\alpha}$. Remark that Lemma 4.5 and (9) imply that, as $\alpha \rightarrow 1$,

$$
\frac{R_{\alpha}}{c_{\alpha} r_{\alpha}} \rightarrow 8 \pi
$$

Similar to (44), equation (50) may be rewritten as

$$
-L_{\alpha} \eta_{\alpha}=\frac{\varphi^{\prime}\left(c_{\alpha}\right)}{\left(1+R_{\alpha}^{-2}\left|\nabla \eta_{\alpha}\right|^{2}\right)^{\alpha-1}} r_{\alpha}^{2} f\left(v_{\alpha}\right)=V_{\alpha} e^{\eta_{\alpha}}
$$

where

$$
\begin{aligned}
0<V_{\alpha} & =\frac{\varphi^{\prime}\left(c_{\alpha}\right)}{v_{\alpha}\left(1+R_{\alpha}^{-2}\left|\nabla \eta_{\alpha}\right|^{2}\right)^{\alpha-1}} W_{\alpha} \\
& =\frac{\varphi^{\prime}\left(c_{\alpha}\right)}{c_{\alpha}\left(1+r_{\alpha}^{-2}\left|\nabla w_{\alpha}\right|^{2}\right)^{\alpha-1}} \exp \left(R_{\alpha}\left(c_{\alpha}, w_{\alpha}\right)+\log \left(1+\frac{w_{\alpha}}{c_{\alpha}}\right)\right) \\
& \leq 8 \pi\left(1+\frac{w_{\alpha}}{c_{\alpha}}\right) e^{4 \pi w_{\alpha}^{2}} .
\end{aligned}
$$

Lemma 4.6. For any $r>0$ there holds

$$
\int_{B_{r}(y)} e^{\eta_{\alpha}} d x \leq 2 \int_{B_{r}(y)} r_{\alpha}^{2} v_{\alpha} f\left(v_{\alpha}\right) d x \leq C
$$

and

$$
\int_{B_{r}(y)} e^{\varphi\left(c_{\alpha}\right)+2 \log \left(c_{\alpha} r_{\alpha}\right)} d x \leq \int_{B_{r}(y)} r_{\alpha}^{2} v_{\alpha} f\left(v_{\alpha}\right) d x \leq C,
$$

uniformly in $\alpha>1$.

Proof. The function $h: s \rightarrow s^{2} e^{\varphi(s)}$ being convex for $s \geq 0$, Jensen's inequality implies that

$$
\begin{aligned}
c_{\alpha}^{2} e^{\varphi\left(c_{\alpha}\right)}=h\left(c_{\alpha}\right) \leq f_{B_{r}(y)} h\left(v_{\alpha}\right) d x & =f_{B_{r}(y)} v_{\alpha}^{2} e^{\varphi\left(v_{\alpha}\right)} d x \\
& =r_{\alpha}^{-2} f_{B_{r}(y)} r_{\alpha}^{2} v_{\alpha} f\left(v_{\alpha}\right) d x
\end{aligned}
$$


that is,

$$
\int_{B_{r}(y)} e^{\varphi\left(c_{\alpha}\right)+2 \log \left(c_{\alpha} r_{\alpha}\right)} d x \leq \int_{B_{r}(y)} r_{\alpha}^{2} v_{\alpha} f\left(v_{\alpha}\right) d x,
$$

proving (54).

Observe that $\eta_{\alpha} \leq \varphi\left(c_{\alpha}\right)+2 \log \left(c_{\alpha} r_{\alpha}\right)$ whenever $w_{\alpha}(x) \leq 0$. Moreover, on the set where $w_{\alpha}(x) \geq 0$ we have $W_{\alpha}(x) \geq 1$ and hence

$$
\int_{B_{r}(y)} e^{\eta_{\alpha}} d x \leq \int_{B_{r}(y)}\left(e^{\varphi\left(c_{\alpha}\right)+2 \log \left(c_{\alpha} r_{\alpha}\right)}+W_{\alpha} e^{\eta_{\alpha}}\right) d x \leq 2 \int_{B_{r}(y)} r_{\alpha}^{2} v_{\alpha} f\left(v_{\alpha}\right) d x .
$$

Lemma 4.7. For any $r \leq 1$ there holds $\sup _{B_{r / 8}(y)} \eta_{\alpha} \leq C$, uniformly in $\alpha$.

Proof. We adapt the proof of [7], Corollary 4, to our setting. Clearly it suffices to consider the case $r=1$; however, we keep the general notation $r$ in order to facilitate the reading. Split $\eta_{\alpha}=\eta_{1 \alpha}+\eta_{2 \alpha}$, where $\eta_{1 \alpha}$ solves

$$
-\operatorname{div}\left(\left(1+R_{\alpha}^{-2}\left|\nabla \eta_{\alpha}\right|^{2}\right)^{\alpha-1} \nabla \eta_{1 \alpha}\right)=\varphi^{\prime}\left(c_{\alpha}\right) r_{\alpha}^{2} f\left(v_{\alpha}\right) \text { in } B_{r}(y)
$$

with $\eta_{1 \alpha}=0$ on $\partial B_{r}(y)$. Observe that

$$
0 \leq \varphi^{\prime}\left(c_{\alpha}\right) r_{\alpha}^{2} f\left(v_{\alpha}\right) \leq 8 \pi r_{\alpha}^{2} c_{\alpha} v_{\alpha} e^{\varphi\left(v_{\alpha}\right)} \leq 8 \pi r_{\alpha}^{2} \max \left\{v_{\alpha}^{2} e^{\varphi\left(v_{\alpha}\right)}, c_{\alpha}^{2} e^{\varphi\left(c_{\alpha}\right)}\right\} .
$$

Hence from Lemma 4.6 and our normalization condition we conclude that

$$
\left\|\varphi^{\prime}\left(c_{\alpha}\right) r_{\alpha}^{2} f\left(v_{\alpha}\right)\right\|_{L^{1}\left(B_{r}(y)\right)} \leq 8 \pi \varepsilon .
$$

In view of Theorem 2.2 then $e^{\eta_{1 \alpha}} \in L^{p}\left(B_{r}(y)\right)$ for any $p<\infty$, provided $\varepsilon=\varepsilon(p)$ has been fixed sufficiently small. In addition, $\nabla \eta_{1 \alpha} \in L^{q}\left(B_{r}(y)\right)$ for any $q<2$, with

$$
\left\|\nabla \eta_{1 \alpha}\right\|_{L^{q}} \leq C(q) .
$$

Finally, $\eta_{1 \alpha} \geq 0$ by the weak maximum principle for (55).

On the other hand, $\eta_{2 \alpha}$ satisfies

$$
-\operatorname{div}\left(\left(1+R_{\alpha}^{-2}\left|\nabla \eta_{\alpha}\right|^{2}\right)^{\alpha-1} \nabla \eta_{2 \alpha}\right)=0 \text { in } B_{r}(y),
$$

and we may expect $\eta_{2 \alpha}$ to have good local regularity properties in $B_{r}(y)$. Because of the coupling of the coefficient $b_{\alpha}:=\left(1+R_{\alpha}^{-2}\left|\nabla \eta_{\alpha}\right|^{2}\right)^{\alpha-1} \geq 1$ in (56) to the gradient of the solution $\eta_{2 \alpha}$, however, these estimates are somewhat delicate.

Fix $\psi(x)=\min \{1,2-2|x-y| / r\} \in W_{0}^{1, \infty}\left(B_{r}(y)\right)$ as cut-off function. (The reason for this particular choice will only become apparent at the end of the proof.) Multiply (56) by $\psi$ to obtain

$0=-\operatorname{div}\left(b_{\alpha} \nabla \eta_{2 \alpha}\right) \psi=-\operatorname{div}\left(b_{\alpha} \nabla\left(\eta_{2 \alpha} \psi\right)\right)+\operatorname{div}\left(b_{\alpha} \eta_{2 \alpha} \nabla \psi\right)+b_{\alpha} \nabla \eta_{2 \alpha} \nabla \psi$ 
Multiplying by $\left(\eta_{2 \alpha}\right)_{+} \psi^{2 \alpha-1}$ and integrating by parts, we conclude

$$
\begin{gathered}
\int_{B_{r}(y)} b_{\alpha}\left|\nabla\left(\eta_{2 \alpha} \psi\right)_{+}\right|^{2} \psi^{2(\alpha-1)} d x=(2 \alpha-1) \int_{B_{r}(y)} b_{\alpha}|\nabla \psi|^{2} \eta_{2 \alpha+}^{2} \psi^{2(\alpha-1)} d x \\
-2(\alpha-1) \int_{B_{r}(y)} b_{\alpha} \nabla\left(\eta_{2 \alpha} \psi\right)_{+} \nabla \psi \eta_{2 \alpha+} \psi^{2(\alpha-1)} d x .
\end{gathered}
$$

Using the estimate $2 a b \leq \frac{1}{2} a^{2}+2 b^{2}$, for $1<\alpha \leq 2$ the last term may be absorbed in the remaining two, which yields

$\frac{1}{2} \int_{B_{r}(y)} b_{\alpha}\left|\nabla\left(\eta_{2 \alpha} \psi\right)_{+}\right|^{2} \psi^{2(\alpha-1)} d x \leq(2 \alpha+1) \int_{B_{r}(y)} b_{\alpha}|\nabla \psi|^{2} \eta_{2 \alpha+}^{2} \psi^{2(\alpha-1)} d x$.

Since $b_{\alpha} \geq 1$, therefore we obtain

$$
I:=\int_{B_{r}(y)}\left|\nabla\left(\eta_{2 \alpha} \psi\right)_{+}\right|^{2} \psi^{2(\alpha-1)} d x \leq 10 \int_{B_{r}(y)} b_{\alpha}|\nabla \psi|^{2} \eta_{2 \alpha+}^{2} \psi^{2(\alpha-1)} d x .
$$

Now observe that

$$
b_{\alpha} \psi^{2(\alpha-1)}=\left[\left(1+R_{\alpha}^{-2}\left|\nabla \eta_{\alpha}\right|^{2}\right) \psi^{2}\right]^{\alpha-1} .
$$

Expanding

$$
\left|\nabla\left(\eta_{\alpha} \psi\right)\right|^{2}=\left|\nabla \eta_{\alpha}\right|^{2} \psi^{2}+2 \nabla \eta_{\alpha} \nabla \psi \eta_{\alpha} \psi+\eta_{\alpha}^{2}|\nabla \psi|^{2}
$$

and estimating

$$
-2 \nabla \eta_{\alpha} \nabla \psi \eta_{\alpha} \psi \leq \frac{1}{2}\left|\nabla \eta_{\alpha}\right|^{2} \psi^{2}+2 \eta_{\alpha}^{2}|\nabla \psi|^{2},
$$

we find that

$$
\left|\nabla \eta_{\alpha}\right|^{2} \psi^{2} \leq 2\left|\nabla\left(\eta_{\alpha} \psi\right)\right|^{2}+2 \eta_{\alpha}^{2}|\nabla \psi|^{2} .
$$

By concavity of the function $0<s \mapsto s^{\alpha-1}$, therefore

$$
\begin{aligned}
b_{\alpha} \psi^{2(\alpha-1)} & \leq\left[\psi^{2}+2 R_{\alpha}^{-2}\left(\left|\nabla\left(\eta_{\alpha} \psi\right)\right|^{2}+\eta_{\alpha}^{2}|\nabla \psi|^{2}\right)\right]^{\alpha-1} \\
& \leq \psi^{2(\alpha-1)}+2 R_{\alpha}^{-2(\alpha-1)}\left(\left|\nabla\left(\eta_{\alpha} \psi\right)\right|^{2(\alpha-1)}+\eta_{\alpha}^{2(\alpha-1)}|\nabla \psi|^{2(\alpha-1)}\right) .
\end{aligned}
$$

Since $\eta_{1 \alpha} \geq 0$ we can estimate

$$
\eta_{2 \alpha+}=\left(\eta_{\alpha}-\eta_{1 \alpha}\right)_{+} \leq \eta_{\alpha+}
$$

Also estimating

$$
\left|\nabla\left(\eta_{\alpha} \psi\right)\right|^{2}=\left|\nabla\left(\left(\eta_{1 \alpha} \psi\right)+\left(\eta_{2 \alpha} \psi\right)\right)\right|^{2} \leq 2\left|\nabla\left(\eta_{1 \alpha} \psi\right)\right|^{2}+2\left|\nabla\left(\eta_{2 \alpha} \psi\right)\right|^{2}
$$


from (57) and (58) we obtain that

$$
\begin{aligned}
I \leq & 10 \int_{B_{r}^{+}(y)}|\nabla \psi|^{2} \eta_{\alpha}^{2} \psi^{2(\alpha-1)} d x \\
& +40 \int_{B_{r}^{+}(y)} R_{\alpha}^{-2(\alpha-1)}\left|\nabla\left(\eta_{1 \alpha} \psi\right)\right|^{2(\alpha-1)}|\nabla \psi|^{2} \eta_{\alpha}^{2} d x \\
& +40 \int_{B_{r}^{+}(y)} R_{\alpha}^{-2(\alpha-1)}\left|\nabla\left(\eta_{2 \alpha} \psi\right)_{+}\right|^{2(\alpha-1)}|\nabla \psi|^{2} \eta_{\alpha}^{2} d x \\
& +20 \int_{B_{r}^{+}(y)} R_{\alpha}^{-2(\alpha-1)}|\nabla \psi|^{2 \alpha} \eta_{\alpha}^{2 \alpha} d x,
\end{aligned}
$$

where $B_{r}^{+}(y)=\left\{x \in B_{r}(y) ; \eta_{\alpha}(x) \geq 0\right\}$.

Observe that

$$
-2 \log \left(c_{\alpha} r_{\alpha}\right)=\varphi\left(c_{\alpha}\right)+\varphi^{\prime}\left(c_{\alpha}\right) w_{\alpha}-\eta_{\alpha} \leq \varphi\left(c_{\alpha}\right)+\varphi^{\prime}\left(c_{\alpha}\right) w_{\alpha}
$$

on $B_{r}^{+}(y)$. Since for small $\alpha>1$ on account of Theorem 2.4 and (41) we have

$$
c_{\alpha} \leq \sup _{\Omega}\left|u_{\alpha}\right| \leq \frac{2}{\sqrt{\alpha-1}},
$$

from (51) and (9) we conclude that

$$
\begin{aligned}
R_{\alpha}^{-2(\alpha-1)} & \leq C e^{\left(\varphi\left(c_{\alpha}\right)+\varphi^{\prime}\left(c_{\alpha}\right) w_{\alpha}\right)(\alpha-1)} \\
& \leq C e^{4 \pi c_{\alpha}^{2}(\alpha-1)} \cdot e^{8 \pi c_{\alpha}(\alpha-1)\left|w_{\alpha}\right|} \leq C e^{16 \pi \sqrt{\alpha-1}\left|w_{\alpha}\right|} \leq C e^{\left|w_{\alpha}\right|}
\end{aligned}
$$

on $B_{r}^{+}(y)$, if $\alpha>1$ is sufficiently small. Thus, (59) implies that

$$
\begin{aligned}
I \leq & 10 \int_{B_{r}(y)}|\nabla \psi|^{2} \eta_{\alpha+}^{2} \psi^{2(\alpha-1)} d x \\
& +C \int_{B_{r}(y)}\left|\nabla\left(\eta_{1 \alpha} \psi\right)\right|^{2(\alpha-1)}|\nabla \psi|^{2} \eta_{\alpha+}^{2} e^{\left|w_{\alpha}\right|} d x \\
& +C \int_{B_{r}(y)}\left|\nabla\left(\eta_{2 \alpha} \psi\right)_{+}\right|^{2(\alpha-1)}|\nabla \psi|^{2} \eta_{\alpha+}^{2} e^{\left|w_{\alpha}\right|} d x \\
& +C \int_{B_{r}(y)}|\nabla \psi|^{2 \alpha} \eta_{\alpha+}^{2 \alpha} e^{\left|w_{\alpha}\right|} d x=I I+\ldots+V .
\end{aligned}
$$

Now $\psi \in W^{1, \infty}\left(B_{r}(y)\right)$; moreover, for any fixed $k$ we can estimate $\eta_{\alpha+}^{k} \leq k ! e^{\eta_{\alpha}}$ and by Lemma 4.6 the latter is bounded in $L^{1}\left(B_{r}(y)\right)$ as $\alpha \rightarrow 1$. Since also $e^{\left|w_{\alpha}\right|}$ is bounded, say, in $L^{6}\left(B_{r}(y)\right)$ if $\varepsilon>0$ is chosen sufficiently small, the terms II and V are bounded as $\alpha \rightarrow 1$.

Similarly, the term III is seen to be bounded for sufficiently small $\alpha>1$ in view of the uniform boundedness of $\left|\nabla \eta_{1 \alpha}\right|$ in $L^{q}\left(B_{r}(y)\right)$ for any $q<2$. 
Finally, we show the term IV can be absorbed. It is here where the special choice of $\psi$ is needed. Use Young's inequality $a b \leq \frac{a^{p}}{p}+\frac{b^{q}}{q}$ with

$$
\begin{gathered}
a=\left(\left|\nabla\left(\eta_{2 \alpha} \psi\right)_{+}\right|^{2} \psi^{2(\alpha-1)}\right)^{\alpha-1}, b=\psi^{-2(\alpha-1)^{2}} \eta_{\alpha+}^{2} e^{\left|w_{\alpha}\right|}, \\
p=\frac{1}{\alpha-1}, q=\frac{1}{2-\alpha}
\end{gathered}
$$

to obtain, for sufficiently small $\alpha>1$,

$$
\begin{aligned}
I V & \leq C(\alpha-1) I+C \int_{B_{r}(y)} \psi^{-\frac{2(\alpha-1)^{2}}{2-\alpha}} \eta_{\alpha+}^{\frac{2}{2-\alpha}} e^{\left|w_{\alpha}\right| /(2-\alpha)} d x \\
& \leq \frac{1}{2} I+C \int_{B_{r}(y)} \psi^{-\frac{1}{2}} \eta_{\alpha+}^{2} e^{\left|w_{\alpha}\right|}\left(1+\eta_{\alpha+}^{2}+e^{\left|w_{\alpha}\right|}\right) d x .
\end{aligned}
$$

Since $\psi^{-\frac{1}{2}} \in L^{q}\left(B_{r}(y)\right)$ for $q<2$, it follows that

$$
I V \leq \frac{1}{2} I+C
$$

and hence

$$
C_{1}:=2 \limsup _{\alpha \rightarrow 1} I<\infty .
$$

In particular, for small $\alpha>1$ we obtain

$$
\int_{B_{r / 2}(y)}\left|\nabla \eta_{2 \alpha+}\right|^{2} d x \leq I \leq C_{1}
$$

Let

$$
\bar{\eta}_{2 \alpha+}=f_{B_{r / 2}(y)} \eta_{2 \alpha+} d x
$$

Since $\eta_{2 \alpha+} \leq \eta_{\alpha+}=\max \left\{0, \eta_{\alpha}\right\}$, by Jensen's inequality and Lemma 4.6 we can bound

$$
\begin{aligned}
0 \leq \bar{\eta}_{2 \alpha+} & \leq \log \left(f_{B_{r / 2}(y)} e^{\eta_{2 \alpha+}} d x\right) \\
& \leq \log \left(f_{B_{r / 2}(y)}\left(1+e^{\eta_{\alpha}}\right) d x\right) \leq C=C(r) .
\end{aligned}
$$

Estimating $\eta_{2 \alpha+}^{2} \leq 2\left|\eta_{2 \alpha+}-\bar{\eta}_{2 \alpha+}\right|^{2}+2 \bar{\eta}_{2 \alpha+}^{2}$, from Theorem 2.1 we then obtain a uniform bound for the function

$$
\exp \left(\frac{\pi \eta_{2 \alpha+}^{2}}{C_{1}}\right) \leq C \exp \left(\frac{2 \pi\left|\eta_{2 \alpha+}-\bar{\eta}_{2 \alpha+}\right|^{2}}{C_{1}}\right)
$$

in $L^{1}\left(B_{r / 2}(y)\right)$. 
Given $1<p<\infty$, estimate $p \eta_{2 \alpha+} \leq \frac{\pi \eta_{2 \alpha+}^{2}}{C_{1}}+\frac{C_{1} p^{2}}{4 \pi}$. Also estimating $\eta_{\alpha} \leq \eta_{1 \alpha}+\eta_{2 \alpha}$ and using our information about $\eta_{1 \alpha}$, for sufficiently small $\varepsilon>0$ we then find that $e^{\eta_{\alpha}}$ is bounded in $L^{p}\left(B_{r / 2}(y)\right)$ as $\alpha \rightarrow 1$.

By (53), Lemma 4.2, and Theorem 2.1 also $V_{\alpha}$ is bounded in $L^{p}\left(B_{r / 2}(y)\right)$ as $\alpha \rightarrow 1$ for any given $p<\infty$, if $\varepsilon>0$ is sufficiently small. Choosing $p=4$, by Hölder's inequality we obtain a uniform bound for $L_{\alpha} \eta_{\alpha}=$ $-V_{\alpha} e^{\eta_{\alpha}}$ in $L^{2}\left(B_{r / 2}(y)\right)$.

Since equation (52) is uniformly elliptic, from [8], Theorem 4.8.(2), we obtain the bound

$$
\begin{aligned}
\sup _{B_{r / 8}(y)} \eta_{\alpha} & \leq \sup _{Q_{\rho / 2}(y)} \eta_{\alpha} \leq C\left(\left\|\eta_{\alpha+}\right\|_{L^{1}\left(Q_{\rho}(y)\right)}+\left\|L_{\alpha} \eta_{\alpha}\right\|_{L^{2}\left(Q_{\rho}(y)\right)}\right) \\
& \leq C \int_{B_{r}(y)} e^{\eta_{\alpha}} d x+C\left\|V_{\alpha} e^{\eta_{\alpha}}\right\|_{L^{2}\left(B_{r / 2}(y)\right)} \leq C
\end{aligned}
$$

where $\rho=\frac{r}{2 \sqrt{2}}$ so that $B_{r / 8}(y) \subset Q_{\rho / 2}(y) \subset Q_{\rho}(y) \subset B_{r / 2}(y)$.

Lemma 4.8. Given $p<\infty$, there exists $\varepsilon_{p}>0$ such that $L_{\alpha} v_{\alpha} \rightarrow 0$ in $L_{\text {loc }}^{p}$ as $\alpha \rightarrow 1$, provided $0<\varepsilon<\varepsilon_{p}$.

Proof. For any $R>0, y \in \mathbb{R}^{2}$ cover $B_{R}(y)$ by finitely many balls $B_{r}(z)$ of radius $r=1 / 8$. Given $p<\infty$, by Theorem 2.1 and (53) then $\left(V_{\alpha}\right)_{\alpha>1}$ is bounded in $L^{p}\left(B_{r}(z)\right)$ if $\varepsilon>0$ is sufficiently small, and by Lemma 4.7 the same is true for the family $\left(L_{\alpha} \eta_{\alpha}\right)_{\alpha>1}$. In particular, then $L_{\alpha} v_{\alpha}=L_{\alpha} w_{\alpha}=$ $L_{\alpha} \eta_{\alpha} / \varphi^{\prime}\left(c_{\alpha}\right) \rightarrow 0$ in $L^{p}\left(B_{r}(z)\right)$.

Note that, while the definitions of $w_{\alpha}, \eta_{\alpha}$ and $V_{\alpha}$ a-priori depend on the choice of ball, the expression $L_{\alpha} v_{\alpha}$ is unambiguously defined. Therefore, covering $B_{R}(y)$ as above, we find that $L_{\alpha} v_{\alpha} \rightarrow 0$ in $L^{p}\left(B_{R}(y)\right)$ for any $y$ and $R$, as desired.

In the following we will fix some $p>2$, say, $p=4$, and assume $\varepsilon>0$ has been chosen according to Lemmas 4.4, 4.7, and 4.8.

We return to the task of estimating the oscillatory component $w_{\alpha}$ of $v_{\alpha}$ on a ball $B_{r}(y)$.

Lemma 4.9. For any $r>0$, any $y$, as $\alpha \rightarrow 1$ we have $w_{\alpha} \rightarrow 0$ locally in $C^{1}$ on $B_{r}(y)$.

Proof. For any $r>0$ by Lemma 4.2 we have $w_{\alpha} \rightarrow 0$ weakly in $H^{1}\left(B_{r}(y)\right)$. Moreover, by Lemma 4.8, there holds $L_{\alpha} w_{\alpha}=L_{\alpha} v_{\alpha} \rightarrow 0$ in $L^{p}\left(B_{r}(y)\right)$. For any $\psi \in C_{0}^{\infty}\left(B_{r}(y)\right)$ then the function $w_{\alpha} \psi$ satisfies

$$
L_{\alpha}\left(w_{\alpha} \psi\right)=\psi L_{\alpha} w_{\alpha}+w_{\alpha} L_{\alpha} \psi+2 a_{\alpha}^{i j} \partial_{i} w_{\alpha} \partial_{j} \psi \rightarrow 0
$$

weakly in $L^{2}\left(B_{r}(y)\right)$ as $\alpha \rightarrow 1$. 
Hence $w_{\alpha} \psi \rightarrow 0$ weakly in $H^{2}\left(B_{r}(y)\right)$ and strongly in $W^{1, p}\left(B_{r}(y)\right)$ as $\alpha \rightarrow 0$. Since $\psi$ is arbitrary, therefore $w_{\alpha} \rightarrow 0$ in $W_{l o c}^{1, p}\left(B_{r}(y)\right)$. But then for any $\psi \in C_{0}^{\infty}\left(B_{r}(y)\right)$ we find that $L_{\alpha}\left(w_{\alpha} \psi\right) \rightarrow 0$ strongly in $L^{p}\left(B_{r}(y)\right)$, and we conclude that $w_{\alpha} \psi \rightarrow 0$ in $W^{2, p}\left(B_{r}(y)\right) \hookrightarrow C^{1}\left(\overline{B_{r}(y)}\right)$. Since $\psi$ was arbitrary, the claim follows.

The following lemma is crucial for the exact determination of the concentration energy level.

Lemma 4.10. As $\alpha \rightarrow 1$ there holds $r_{\alpha}^{\alpha-1} \rightarrow 1$, and for any $r>0$, any $y$ we have $V_{\alpha} \rightarrow V_{0} \equiv 8 \pi$ locally uniformly on $B_{r}(y)$.

Proof. Since $w_{\alpha} \rightarrow 0$ locally $C^{1}$-uniformly, by formula (53) for $V_{\alpha}$ it suffices to show that $r_{\alpha}^{\alpha-1} \rightarrow 1$ as $\alpha \rightarrow 1$. This will follow from the "entropy" bound (12) or (40), which we write in the form

$$
0=\liminf _{\alpha \rightarrow 0}\left((\alpha-1) \log \frac{1}{(\alpha-1)} S_{\alpha}\left(u_{\alpha}\right)\right)
$$

where

$$
S_{\alpha}(v)=\int_{\Omega} \log \left(1+|\nabla v|^{2}\right)\left(1+|\nabla v|^{2}\right)^{\alpha} d x .
$$

To derive the desired estimate, we replace $S_{\alpha}\left(u_{\alpha}\right)$ by a more tractable quantity.

Decompose $v_{\alpha}=w_{\alpha}+c_{\alpha}$ on $B_{2}(0)$ and remark that Lemma 4.9 implies

$$
\left|v_{\alpha}(x)-c_{\alpha}\right|=\left|w_{\alpha}(x)\right| \leq 1
$$

for sufficiently small $\alpha>1$, uniformly for $x \in B_{1}(0)$. Similar to our proof of Lemma 4.1 our normalization then implies

$$
0<\frac{\varepsilon}{2} \leq \int_{B_{1}(0)} r_{\alpha}^{2} v_{\alpha} f\left(v_{\alpha}\right) d x \leq \pi r_{\alpha}^{2}\left(c_{\alpha}+1\right)^{2} e^{\varphi\left(c_{\alpha}+1\right)} \leq 2 \pi r_{\alpha}^{2} c_{\alpha}^{2} e^{8 \pi\left(c_{\alpha}^{2}+1\right)} .
$$

Taking logarithms, we find

$$
\log r_{\alpha}^{-1} \leq 4 \pi c_{\alpha}^{2}+\log c_{\alpha}+C \leq 16 c_{\alpha}^{2}
$$

for small $\alpha>1$, and hence

$$
\inf _{B_{r_{\alpha}\left(x_{\alpha}\right)}} u_{\alpha} \geq c_{\alpha}-1 \geq \frac{1}{4} \sqrt{\left|\log r_{\alpha}\right|}-1=: \gamma_{\alpha} .
$$

Fix $R>0$ such that $\mu\left(B_{R}(0)\right)=\mu(\Omega)$ and for $v \in H_{0}^{1}\left(B_{R}(0)\right)$ let

$$
S_{\alpha}^{*}(v)=\int_{B_{R} \backslash B_{r_{\alpha}}(0)} \log \left(1+|\nabla v|^{2}\right)\left(1+|\nabla v|^{2}\right)^{\alpha} d x \leq \infty .
$$


Note that the function $s \mapsto h(s)=\left(1+s^{2}\right)^{\alpha} \log \left(1+s^{2}\right)$ is convex and non-negative for $s \geq 0$. Hence, by Schwarz symmetrization

$$
S_{\alpha}\left(u_{\alpha}\right) \geq \int_{B_{R}(0)} \log \left(1+\left|\nabla u_{\alpha}^{*}\right|^{2}\right)\left(1+\left|\nabla u_{\alpha}^{*}\right|^{2}\right)^{\alpha} d x \geq S_{\alpha}^{*}\left(u_{\alpha}^{*}\right),
$$

where $u_{\alpha}^{*}$ is the radially decreasing rearrangement of $u_{\alpha}$ on $B_{R}(0)=\Omega^{*}$. See for instance [15], p. 91; Corollary 2.33 and its proof easily may be carried over to our setting.

From (61) we then derive

$$
S_{\alpha}\left(u_{\alpha}\right) \geq S_{\alpha}^{*}\left(u_{\alpha}^{*}\right) \geq S_{\alpha}^{*}\left(\min \left\{u_{\alpha}^{*}, \gamma_{\alpha}\right\}\right) \geq \inf _{v \in N_{\alpha}} S_{\alpha}^{*}(v)
$$

where

$$
N_{\alpha}=\left\{v \in H_{0}^{1}\left(B_{R}(0)\right) ; v=\gamma_{\alpha} \text { on } B_{r_{\alpha}}(0)\right\} .
$$

Note that $N_{\alpha}$ is weakly sequentially closed while $S_{\alpha}^{*}$ is coercive and weakly lower semi-continuous on $N_{\alpha}$. Hence there exists a radially symmetric minimizer $v^{*}=v_{\alpha}^{*} \in N_{\alpha}$ for $S_{\alpha}^{*}$. Letting $v^{*}=v^{*}(r)$ with radial derivative $v_{r}^{*}$, then $v^{*}$ solves the boundary value problem

$$
\left[r\left(1+\alpha \log \left(1+\left|v_{r}^{*}\right|^{2}\right)\right)\left(1+\left|v_{r}^{*}\right|^{2}\right)^{\alpha-1} v_{r}^{*}\right]_{r}=0
$$

for $r_{\alpha}<r<R$ with data

$$
v^{*}(R)=0, v^{*}\left(r_{\alpha}\right)=\gamma_{\alpha} .
$$

From (63), (64) we deduce that for some $C_{2}>0$ we can estimate

$$
S_{\alpha}^{*}\left(v_{\alpha}^{*}\right) \geq C_{2} \frac{\left(\log r_{\alpha}^{-1}\right)^{\frac{1}{2 \alpha-1}}}{\log \log r_{\alpha}^{-1}},
$$

uniformly as $\alpha \rightarrow 1$. This somewhat technical estimate is given separately at the end of this proof. By Lemma 4.1, moreover, we have

$$
\log r_{\alpha}^{-1} \leq \frac{C}{\alpha-1}
$$

Combining (60), (62), and (65), we then conclude that

$$
\begin{aligned}
0 & =\liminf _{\alpha \rightarrow 1}\left((\alpha-1) \log \left(\frac{1}{\alpha-1}\right) \frac{\left(\log r_{\alpha}^{-1}\right)^{\frac{1}{\alpha-1}}}{\log \log r_{\alpha}^{-1}}\right) \\
& \geq \liminf _{\alpha \rightarrow 1}\left((\alpha-1)\left(\log r_{\alpha}^{-1}\right)^{\frac{1}{\alpha-1}}\right) \\
& =\liminf _{\alpha \rightarrow 1}\left((\alpha-1) \log r_{\alpha}^{-1}\right)^{\frac{1}{2 \alpha-1}}=\liminf _{\alpha \rightarrow 1}\left(\log r_{\alpha}^{-(\alpha-1)}\right)^{\frac{1}{2 \alpha-1}},
\end{aligned}
$$

and hence $r_{\alpha}^{\alpha-1} \rightarrow 1$, as desired. It remains to show (65). 
Proof of (65): We write $v$ instead of $v^{*}$ for simplicity. By (63) there exists a constant $K=K_{\alpha}$ such that

$$
r\left(1+\alpha \log \left(1+v_{r}^{2}\right)\right)\left(1+v_{r}^{2}\right)^{\alpha-1} v_{r} \equiv-K
$$

for $r_{\alpha}<r<R$. In view of (64) it follows that $K>0, v_{r}<0$, and $v_{r}$ is strictly increasing.

Suppose $v_{r}(R) \geq-2$. Since $\gamma_{\alpha} \rightarrow \infty$ as $\alpha \rightarrow 1$, for sufficiently small $\alpha>1$ there exists $\left.\left.\tilde{r}_{\alpha} \in\right] r_{\alpha}, R\right]$ such that $v_{r}\left(\tilde{r}_{\alpha}\right)=-2$ and $\left|v_{r}(r)\right| \geq 2$ for $r_{\alpha}<r<\tilde{r}_{\alpha}$ while $\left|v_{r}(r)\right| \leq 2$ for $\tilde{r}_{\alpha} \leq r \leq R$. If $v_{r}(R) \leq-2$, we let $\tilde{r}_{\alpha}=R$.

It follows that $v\left(\tilde{r}_{\alpha}\right) \leq 2 R \leq 2 R_{0}$ is uniformly bounded. Moreover, by (66) for $r_{\alpha}<r<\tilde{r}_{\alpha}$ we obtain

$$
\left|v_{r}\right|^{2 \alpha-1} \approx \frac{K}{r \log \left|v_{r}\right|} \lesssim \frac{K}{r}
$$

where we write $a \approx b$ if $C^{-1} a \leq b \leq C a$ with a uniform constant $C$ independent of $r$ and $\alpha$. Similarly, we write $a \lesssim b$ if $a \leq C b$. It follows that

$$
\frac{K}{r \log \left(\frac{K}{r}\right)} \lesssim \frac{K}{r \log \left|v_{r}\right|} \approx\left|v_{r}\right|^{2 \alpha-1}
$$

and hence also

$$
\frac{K}{r \log \left|v_{r}\right|} \lesssim \frac{K}{r \log \left(\frac{K}{r}\right)}
$$

that is,

$$
\left|v_{r}\right| \approx\left(\frac{K}{r \log \left(\frac{K}{r}\right)}\right)^{\frac{1}{2 \alpha-1}} .
$$

We also find

$$
\gamma_{\alpha} \geq \int_{r_{\alpha}}^{\tilde{r}_{\alpha}}\left|v_{r}\right| d r \geq \gamma_{\alpha}-2 R_{0} \geq \frac{1}{2} \gamma_{\alpha}
$$

and therefore

$$
S_{\alpha}^{*}\left(v^{*}\right) \geq 2 \pi \int_{r_{\alpha}}^{\tilde{r}_{\alpha}} r \log \left(1+v_{r}^{2}\right)\left(1+v_{r}^{2}\right)^{\alpha} d r \gtrsim \int_{r_{\alpha}}^{\tilde{r}_{\alpha}} K\left|v_{r}\right| d r \gtrsim \gamma_{\alpha} K
$$

The proof will be complete if we show that

$$
K=K_{\alpha} \gtrsim\left(\log r_{\alpha}^{-1}\right)^{\frac{1}{2 \alpha-1}-\frac{1}{2}} / \log \log r_{\alpha}^{-1} .
$$


Let $\frac{1}{2 \alpha-1}=1-\beta$, where $\beta=\frac{2(\alpha-1)}{2 \alpha-1}>0$. Changing variables $s=\frac{r}{K}=$ $e^{-t}$, from (67) and (68) we obtain

$$
\begin{aligned}
\gamma_{\alpha} & \approx \int_{r_{\alpha}}^{\tilde{r}_{\alpha}}\left(\frac{K}{r \log \left(\frac{K}{r}\right)}\right)^{1-\beta} d r=K \int_{s_{\alpha}}^{\tilde{s}_{\alpha}} \frac{d s}{\left(s \log s^{-1}\right)^{1-\beta}} \\
& =K \int_{\tilde{t}_{\alpha}}^{t_{\alpha}} e^{-\beta t} t^{\beta-1} d t=K \beta^{-\beta} \int_{\beta \tilde{t}_{\alpha}}^{\beta t_{\alpha}} e^{-t} t^{\beta-1} d t,
\end{aligned}
$$

where $s_{\alpha}=\frac{r_{\alpha}}{K}=e^{-t_{\alpha}}, \tilde{s}_{\alpha}=\frac{\tilde{r}_{\alpha}}{K}=e^{-\tilde{t}_{\alpha}}$. Estimating $e^{-t} \lesssim 1, \beta^{-\beta} \lesssim 1$, then we obtain

$$
\begin{aligned}
\sqrt{\left|\log r_{\alpha}\right|} & \lesssim \gamma_{\alpha} \lesssim K \int_{\beta \tilde{t}_{\alpha}}^{\beta t_{\alpha}} t^{\beta-1} d t=K \frac{\left(\beta t_{\alpha}\right)^{\beta}-\left(\beta \tilde{t}_{\alpha}\right)^{\beta}}{\beta} \lesssim K t_{\alpha}^{\beta} \frac{1-\left(\tilde{t}_{\alpha} / t_{\alpha}\right)^{\beta}}{\beta} \\
& \lesssim K t_{\alpha}^{\beta}\left|\log \tilde{t}_{\alpha} / t_{\alpha}\right|=K\left(\log \left(\frac{K}{r_{\alpha}}\right)\right)^{\beta}\left(\log \log \frac{K}{r_{\alpha}}-\log \log \frac{K}{\tilde{r}_{\alpha}}\right) .
\end{aligned}
$$

In particular, $K \rightarrow \infty$ as $\alpha \rightarrow 1$; moreover, either $K \geq \sqrt{\left|\log r_{\alpha}\right|}$, or

$$
\sqrt{\left|\log r_{\alpha}\right|} \lesssim K\left(\log r_{\alpha}^{-1}\right)^{\beta} \log \log r_{\alpha}^{-1} .
$$

Hence we obtain (69) and thus (65).

In particular, Lemma 4.9 implies that for any domain $D \subset \subset \mathbb{R}^{2}$, any $x_{0} \in D$, and any $R_{0}>0$ the mean values $c_{\alpha}(y, r)$, where $y \in D$ and $0<r<R_{0}$, and the value of $v_{\alpha}$ at $y$, or even at the fixed point $x_{0}$, agree up to an error $o(1) \rightarrow 0$ as $\alpha \rightarrow 1$.

It follows that

$$
\eta_{\alpha}=\varphi\left(c_{\alpha}\right)+\varphi_{\alpha}^{\prime}\left(c_{\alpha}\right) w_{\alpha}+2 \log \left(c_{\alpha} r_{\alpha}\right)=\varphi\left(v_{\alpha}\right)+2 \log \left(r_{\alpha} v_{\alpha}\left(x_{0}\right)\right)+o(1),
$$

where $o(1) \rightarrow 0$ in $C^{1}\left(B_{r}(y)\right)$.

Choosing a suitable sequence $R=R(\alpha) \rightarrow \infty$ as $\alpha \rightarrow 1$ and renaming $\eta_{\alpha}=\eta_{\alpha}^{R(\alpha)}$, where $\eta_{\alpha}, \eta_{\alpha}^{R}$ are defined by (49) relative to the decomposition $v_{\alpha}=w_{\alpha}^{R}+c_{\alpha}^{R}$ on $B_{R}(0)$, we then obtain a sequence $\eta_{\alpha}$ which is well-defined on any domain $D \subset \subset \mathbb{R}^{2}$ for sufficiently small $\alpha>1$ and such that

$$
\eta_{\alpha}=\varphi_{\alpha}\left(v_{\alpha}\right)+2 \log \left(r_{\alpha} v_{\alpha}\left(x_{0}\right)\right)+o(1)
$$

with error $o(1) \rightarrow 0$ in $C^{1}(D)$ as $\alpha \rightarrow 1$, where $x_{0} \in \mathbb{R}^{2}$ is fixed arbitrarily. Moreover, we can achieve that $V_{\alpha} \rightarrow V_{0} \equiv 8 \pi$ and $W_{\alpha} \rightarrow W_{0} \equiv 1$ locally uniformly on $\mathbb{R}^{2}$ for this choice of $R(\alpha)$. Similarly, we have the representation $\eta_{\alpha}=\eta_{\alpha}^{(1)}+o(1)$ with error $o(1) \rightarrow 0$ locally $C^{1}$-uniformly as $\alpha \rightarrow 1$, where

$$
\eta_{\alpha}^{(1)}=\varphi_{\alpha}\left(v_{\alpha}\right)+2 \log \left(r_{\alpha} v_{\alpha}\right),
$$

which will be useful later. 
Lemma 4.11. For any $D \subset \subset \mathbb{R}^{2}$ we have $\eta_{\alpha} \in W^{2, p}(D)$ for sufficiently small $\alpha>1$ and $(\alpha-1)\left\|\nabla^{2} \eta_{\alpha}\right\|_{L^{p}(D)} \rightarrow 0$ as $\alpha \rightarrow 1$.

Proof. In view of [8], Theorem 7.1, equations (52) and (44), and taking account of Lemma 4.7, for any ball $B_{r}(y)$ containing $D$ we have

$$
\begin{aligned}
& \left\|\nabla^{2} \eta_{\alpha}\right\|_{L^{p}\left(B_{r}(y)\right)}=\varphi^{\prime}\left(c_{\alpha}\right)\left\|\nabla^{2} w_{\alpha}\right\|_{L^{p}\left(B_{r}(y)\right)} \leq 8 \pi c_{\alpha}\left\|w_{\alpha}\right\|_{W^{2, p}\left(B_{r}(y)\right)} \\
& \quad \leq C\left(c_{\alpha}\left\|w_{\alpha}\right\|_{L^{\infty}\left(B_{2 r}(y)\right)}+c_{\alpha}\left\|L_{\alpha} v_{\alpha}\right\|_{L^{p}\left(B_{2 r}(y)\right)}\right) \\
& \quad \leq o(1) c_{\alpha}+C\left\|L_{\alpha} \eta_{\alpha}\right\|_{L^{p}\left(B_{2 r}(y)\right)} \leq o(1) c_{\alpha}+C
\end{aligned}
$$

where $o(1) \rightarrow 0$ as $\alpha \rightarrow 1$. But Theorem 2.4 implies that $(\alpha-1) c_{\alpha} \rightarrow 0$ as $\alpha \rightarrow 1$, completing the proof.

The $L^{p}$-estimate for $\nabla^{2} \eta_{\alpha}$ from Lemma 4.11 allows to interpret equation (52) as

$$
-\Delta \eta_{\alpha}=V_{\alpha} e^{\eta_{\alpha}}+h_{\alpha} \text { on } \mathbb{R}^{2}
$$

with an error term $h_{\alpha} \rightarrow 0$ in $L_{\text {loc }}^{p}\left(\mathbb{R}^{2}\right)$ as $\alpha \rightarrow 1$. We can remove this error term as in [7], Remark 4. For any large $R>0$ let $\eta_{1 \alpha}$ solve

$$
-\Delta \eta_{1 \alpha}=h_{\alpha} \text { on } B_{R}(0), \eta_{1 \alpha}=0 \text { on } \partial B_{R}(0) .
$$

Observe that $\eta_{1 \alpha} \rightarrow 0$ in $W^{2, p}\left(B_{R}(0)\right)$ as $\alpha \rightarrow 1$ and hence also uniformly on $B_{R}(0)$.

The function $\eta_{2 \alpha}=\eta_{\alpha}-\eta_{1 \alpha}$ then solves

$$
-\Delta \eta_{2 \alpha}=V_{\alpha} e^{\eta_{\alpha}}=\left(V_{\alpha} e^{\eta_{1 \alpha}}\right) e^{\eta_{2 \alpha}} \text { on } B_{R}(0)
$$

and, as $\alpha \rightarrow 1$, again $V_{2 \alpha}=V_{\alpha} e^{\eta_{1 \alpha}} \rightarrow V_{0} \equiv 8 \pi$ uniformly on $B_{R}(0)$.

Replacing $\eta_{\alpha}$ by $\eta_{2 \alpha}$ and $V_{\alpha}$ by $V_{2 \alpha}$ for a suitable sequence $R=$ $R(\alpha) \rightarrow \infty$, we can now invoke the result [7], Theorem 3, and its improvement [17], Theorem, p. 1256, to conclude that one of the following must occur. As $\alpha \rightarrow 1$, either

a) $\eta_{\alpha} \rightarrow-\infty$ locally uniformly on $\mathbb{R}^{2}$; or

b) there are points $x_{1}, \ldots, x_{L} \in \mathbb{R}^{2}$ and numbers $m_{1}, \ldots, m_{L} \in \mathbb{N}$ such that $\eta_{\alpha} \rightarrow-\infty$ locally uniformly on $\mathbb{R}^{2} \backslash\left\{x_{1}, \ldots, x_{L}\right\}$ and $V_{\alpha} e^{\eta_{\alpha}} d x \rightarrow$ $\sum_{l=1}^{L} 4 \pi m_{l} \delta_{x_{l}}$ weakly in the sense of measures; or

c) $\eta_{\alpha} \rightarrow \eta$ locally uniformly in $C^{1, \delta}$ for any $\delta<1$.

Cases a) and b) are ruled out by our normalization (43) and Lemma 4.7.

Thus, only possiblity c) remains; that is, $\eta_{\alpha} \rightarrow \eta=\eta^{(1)}$ in $C^{1, \delta}$, where $\eta$ solves the equation

$$
-\Delta \eta=V_{0} e^{\eta}=8 \pi e^{\eta} \text { on } \mathbb{R}^{2}
$$


with

$$
\begin{aligned}
\int_{\mathbb{R}^{2}} e^{\eta} d x=\lim _{L \rightarrow \infty} \lim _{\alpha \rightarrow 1} \int_{B_{L}(0)} e^{\eta_{\alpha}} d x=\lim _{L \rightarrow \infty} \lim _{\alpha \rightarrow 1} \int_{B_{L}(0)} W_{\alpha} e^{\eta_{\alpha}} d x \\
=\lim _{L \rightarrow \infty} \lim _{\alpha \rightarrow 1} \int_{B_{L r_{\alpha}\left(x_{\alpha}\right)}} u_{\alpha} f\left(u_{\alpha}\right) d x \leq \lim _{\alpha \rightarrow 1} \int_{\Omega} u_{\alpha} f\left(u_{\alpha}\right) d x=2 \beta<\infty .
\end{aligned}
$$

By the classification of such solutions to (71) due to Chen-Li [10] then there exist $x_{0} \in \mathbb{R}^{2}, r_{0}>0$ such that

$$
\eta\left(x_{0}+r_{0} x / \sqrt{V_{0}}\right)+2 \log r_{0}=\log \frac{1}{\left(1+|x|^{2} / 8\right)^{2}}=: \eta_{0}(x) .
$$

In particular, using the fact that $V_{0}=8 \pi$ we may compute

$$
\int_{\mathbb{R}^{2}} e^{\eta} d x=\frac{1}{V_{0}} \int_{\mathbb{R}^{2}} e^{\eta_{0}} d x=1
$$

Moreover, redefining

$$
\tilde{x}_{\alpha}=x_{\alpha}+r_{\alpha} x_{0}, \tilde{r}_{\alpha}=r_{\alpha} r_{0} / \sqrt{V_{0}}, \tilde{v}_{\alpha}=v_{\alpha}+2 \log r_{0},
$$

from (70) we obtain that with error $o(1) \rightarrow 0$ as $\alpha \rightarrow 1$

$$
\begin{aligned}
\tilde{\eta}_{\alpha}(x): & =\varphi\left(u_{\alpha}\left(\tilde{x}_{\alpha}+\tilde{r}_{\alpha} x\right)\right)+2 \log \left(\tilde{r}_{\alpha} u_{\alpha}\left(\tilde{x}_{\alpha}\right)\right)+\log (8 \pi) \\
& =\eta_{\alpha}\left(x_{0}+r_{0} x / \sqrt{V_{0}}\right)+2 \log r_{0}+o(1) \rightarrow \eta_{0}(x)
\end{aligned}
$$

locally $C^{1}$-uniformly on $\mathbb{R}^{2}$, thus proving the last assertion in Theorem 1.3.

\subsection{Energy estimate}

It remains to show that $\beta=\frac{1}{2}$. We show how the argument from [5] may be adapted to the quasilinear case. The proof of the following lemma is almost identical to that of [5], Lemma 5.3, and may be omitted.

Lemma 4.12. There exist radii $t_{\alpha}>0, \alpha>1$, satisfying, as $\alpha \searrow 1$, the conditions $t_{\alpha} \rightarrow 0, r_{\alpha} / t_{\alpha} \rightarrow 0$, dist $\left(x_{\alpha}, \partial \Omega\right) \geq 2 t_{\alpha}$, and

$$
\int_{\Omega \backslash B_{t_{\alpha}}\left(x_{\alpha}\right)} u_{\alpha} f\left(u_{\alpha}\right) d x \rightarrow 0,
$$

while

$$
\inf _{B_{t_{\alpha}}\left(x_{\alpha}\right)} u_{\alpha} \rightarrow \infty
$$

Moreover,

$$
\limsup _{\alpha \searrow 1} \int_{\Omega \backslash B_{t_{\alpha}}\left(x_{\alpha}\right)}\left|\nabla u_{\alpha}\right|^{2} d x \geq 1
$$


Also the next result may be carried over directly from [5], Lemma 5.4, if we replace $w_{k}$ by $w_{\alpha}$ and the Laplace operator by the operator $L_{\alpha}$ in the equation for $\psi w_{k}$ near the end of the proof.

Consider the family $\eta_{\alpha}^{(1)}$ in the original coordinates; that is, let

$$
\eta_{\alpha}=\eta_{\alpha}^{(0)}=\varphi\left(u_{\alpha}\right)+2 \log \left(r_{\alpha} u_{\alpha}\right), \alpha>1 .
$$

For $r>0, y \in \Omega$ also decompose

$$
u_{\alpha}=w_{\alpha}^{(0)}+c_{\alpha} \text { on } B_{r}(y),
$$

where

$$
c_{\alpha}=c_{\alpha}^{(0)}=c_{\alpha}(y, r)=f_{B_{r}(y)} u_{\alpha} d x .
$$

Lemma 4.13. For any $\delta>0$ there is a constant $C_{1}=C_{1}(\delta)$ such that

$$
\begin{aligned}
& \lim _{L \rightarrow \infty} \limsup _{\alpha \searrow 1} \sup _{y}\left(s^{2} e^{\eta_{\alpha}^{(0)}(y)}\right) \leq \delta, \\
& \lim _{L \rightarrow \infty} \limsup _{\alpha \searrow 1} \sup _{y}\left\|\left(\left|w_{\alpha}^{(0)}\right|+s\left|\nabla w_{\alpha}^{(0)}\right|\right)\right\|_{L^{\infty}\left(B_{s / 10}(y)\right)} \leq \delta,
\end{aligned}
$$

where the supremum is taken with respect to $y \in \Omega \backslash B_{L r_{\alpha}}\left(x_{\alpha}\right)$ such that $u_{\alpha}(y) \geq C_{1}$, with $s=\left|y-x_{\alpha}\right| / 2$ and with $w_{\alpha}^{(0)}=u_{\alpha}-c_{\alpha}(y, s)$.

Combining Lemmas 4.9 and 4.13, it is easy to deduce the following result.

Lemma 4.14. We have $\lim \sup _{\alpha \searrow 1} \sup _{y \in \Omega}\left(\left|y-x_{\alpha}\right|\left|\nabla u_{\alpha}(y)\right|\right) \leq C$.

Proof. Arguing indirectly, suppose that for a sequence of points $y_{\alpha} \in \Omega$ as $\alpha \rightarrow 1$ we have $\left|y_{\alpha}-x_{\alpha}\right|\left|\nabla u_{\alpha}\left(y_{\alpha}\right)\right| \rightarrow \infty$.

If $s_{\alpha}:=\left|y_{\alpha}-x_{\alpha}\right| \leq L r_{\alpha}$ for some $L \in \mathbb{N}$, from Lemma 4.9 as $\alpha \rightarrow 1$ we obtain that

$$
s_{\alpha}\left|\nabla u_{\alpha}\left(y_{\alpha}\right)\right| \leq L\left\|\nabla w_{\alpha}^{(1)}\right\|_{L^{\infty}\left(B_{L}(0)\right)} \rightarrow 0 .
$$

Hence $s_{\alpha} / r_{\alpha} \rightarrow \infty$ as $\alpha \rightarrow 1$. Let $C_{1}$ be the constant determined in Lemma 4.13 corresponding to the choice $\delta=1$. If $u_{\alpha} \leq C_{1}$ for some $y \in B_{s_{\alpha} / 30}\left(y_{\alpha}\right)$, letting $s=\left|y-x_{\alpha}\right| / 2$ from Lemma 4.13 we deduce

$$
\limsup _{\alpha \rightarrow 1} s_{\alpha}\left|\nabla u_{\alpha}\left(y_{\alpha}\right)\right| \leq \limsup _{\alpha \rightarrow 1} 3 s\left\|\nabla w_{\alpha}^{(0)}\right\|_{L^{\infty}\left(B_{s / 10}(y)\right)} \leq 3,
$$

thereby observing that

$$
\left|\left(\left|y-x_{\alpha}\right|-\left|y_{\alpha}-x_{\alpha}\right|\right)\right|=\left|2 s-s_{\alpha}\right| \leq\left|y-y_{\alpha}\right| \leq s_{\alpha} / 30
$$

implies that $s_{\alpha} \leq 3 s$ and thus also that $y_{\alpha} \in B_{s / 10}(y)$. 
It remains the case that

$$
\sup _{B_{s_{\alpha} / 30}\left(y_{\alpha}\right)} u_{\alpha} \leq C_{1}
$$

Scaling $\tilde{v}_{\alpha}(x)=u_{\alpha}\left(y_{\alpha}+s_{\alpha} x\right)$, we find that

$$
\begin{aligned}
\tilde{L}_{\alpha} \tilde{v}_{\alpha} & =\Delta \tilde{v}_{\alpha}+2(\alpha-1) \frac{\partial_{i} \tilde{v}_{\alpha} \partial_{i j} \tilde{v}_{\alpha} \partial_{j} \tilde{v}_{\alpha}}{s_{\alpha}^{2}+\left|\nabla \tilde{v}_{\alpha}\right|^{2}} \\
& =-\frac{s_{\alpha}^{2} f\left(\tilde{v}_{\alpha}\right)}{\left(1+s_{\alpha}^{-2}\left|\nabla \tilde{v}_{\alpha}\right|^{2}\right)^{\alpha-1}} \text { in } B_{1 / 30}(0),
\end{aligned}
$$

and from [8], Theorem 7.1, we obtain the uniform bound

$$
s_{\alpha}\left|\nabla u_{\alpha}\left(y_{\alpha}\right)\right|=\left|\nabla \tilde{v}_{\alpha}(0)\right| \leq C\left(\left\|\tilde{L}_{\alpha} \tilde{v}_{\alpha}\right\|_{L^{2}\left(B_{1 / 30}(0)\right)}+\left\|\tilde{v}_{\alpha}\right\|_{L^{\infty}\left(B_{1 / 30}(0)\right)}\right) \leq C .
$$

Thus we arrive at a contradiction, and the proof is complete.

Introducing polar coordinates $(r, \theta)$ around $x_{\alpha}$, we next let

$$
\bar{u}_{\alpha}(r)=f_{\partial B_{r}\left(x_{\alpha}\right)} u_{\alpha} d o
$$

denote the spherical mean of $u_{\alpha}$, etc. We also write $\bar{u}_{\alpha}(x)=\bar{u}_{\alpha}(r)$ for $x \in \partial B_{r}\left(x_{\alpha}\right)$ and denote

$$
w_{\alpha}=u_{\alpha}-\bar{u}_{\alpha}
$$

Expanding $\eta_{\alpha}=\eta_{\alpha}^{(0)}$ around $\bar{u}_{\alpha}$, for $x \in B_{t_{\alpha}}\left(x_{\alpha}\right)$ we find

$$
\eta_{\alpha}-\bar{\eta}_{\alpha}=\left(\varphi^{\prime}\left(\bar{u}_{\alpha}\right)+\frac{2}{\bar{u}_{\alpha}}\right) w_{\alpha}+R\left(\bar{u}_{\alpha}, w_{\alpha}\right)-\overline{R\left(\bar{u}_{\alpha}, w_{\alpha}\right)}
$$

where $(4 \pi-o(1)) w_{\alpha}^{2} \leq R\left(\bar{u}_{\alpha}, w_{\alpha}\right) \leq 4 \pi w_{\alpha}^{2}$ with error $o(1) \rightarrow 0$ uniformly on $B_{t_{\alpha}}\left(x_{\alpha}\right)$ as $\alpha \searrow 1$. Thus, as in [5], formula (26), from Lemmas 4.12 and 4.13 we derive the estimate

$$
0 \leq \int_{\partial B_{r}\left(x_{\alpha}\right)}\left(e^{\eta_{\alpha}}-\eta^{\bar{\eta}_{\alpha}}\right) d o \leq o(1) \int_{\partial B_{r}\left(x_{\alpha}\right)} \bar{u}_{\alpha}^{2}\left|\nabla w_{\alpha}\right|^{2} d o,
$$

where $o(1) \rightarrow 0$ uniformly for $r \in\left[L r_{\alpha}, t_{\alpha}\right]$ and $1<\alpha \leq \alpha(L)$, where $\alpha(L) \rightarrow 1$, as $L \rightarrow \infty$.

Moreover, again using Lemmas 4.12 and 4.13, we have

$$
\begin{gathered}
\int_{\partial B_{r}\left(x_{\alpha}\right)}\left|\nabla\left(\eta_{\alpha}-\bar{\eta}_{\alpha}\right)\right|^{2} d o \leq C \int_{\partial B_{r}\left(x_{\alpha}\right)}\left(\left(\bar{u}_{\alpha}^{2}+w_{\alpha}^{2}\right)\left|\nabla w_{\alpha}\right|^{2}+w_{\alpha}^{2}\left|\nabla \bar{u}_{\alpha}\right|^{2}\right) d o \\
\leq C \int_{\partial B_{r}\left(x_{\alpha}\right)} \bar{u}_{\alpha}^{2}\left|\nabla w_{\alpha}\right|^{2} d o+C \max _{\partial B_{r}\left(x_{\alpha}\right)} w_{\alpha}^{2} \int_{\partial B_{r}\left(x_{\alpha}\right)}\left|\nabla u_{\alpha}\right|^{2} d o \\
\leq C \int_{\partial B_{r}\left(x_{\alpha}\right)} \bar{u}_{\alpha}^{2}\left|\nabla w_{\alpha}\right|^{2} d o+o(1) \int_{\partial B_{r}\left(x_{\alpha}\right)}\left|\nabla u_{\alpha}\right|^{2} d o
\end{gathered}
$$


where $o(1) \rightarrow 0$ uniformly for $r \in\left[L r_{\alpha}, t_{\alpha}\right]$ and $1<\alpha \leq \alpha(L)$ where $\alpha(L) \rightarrow 1$, as $L \rightarrow \infty$. We conclude that

$$
\int_{B_{t_{\alpha}}\left(x_{\alpha}\right)}\left|\nabla\left(\eta_{\alpha}-\bar{\eta}_{\alpha}\right)\right|^{2} d x \leq C \int_{B_{t_{\alpha}}\left(x_{\alpha}\right)} \bar{u}_{\alpha}^{2}\left|\nabla w_{\alpha}\right|^{2} d x+o(1)
$$

with error $o(1) \rightarrow 0$ as $\alpha \rightarrow 1$.

Similarly, from (74), with a uniform constant $C$ and error $o(1) \rightarrow 0$ as $\alpha \rightarrow 1$, for any $r \leq t_{\alpha}$ we obtain

$$
\int_{B_{r}\left(x_{\alpha}\right)} \bar{u}_{\alpha}^{2}\left|\nabla w_{\alpha}\right|^{2} d x \leq C \int_{B_{r}\left(x_{\alpha}\right)}\left|\nabla\left(\eta_{\alpha}-\bar{\eta}_{\alpha}\right)\right|^{2} d x+o(1) .
$$

As in [5], Lemma 5.5, we then deduce the following bound.

Lemma 4.15. $\lim \sup _{\alpha \rightarrow 1} \int_{B_{t_{\alpha}}\left(x_{\alpha}\right)} \bar{u}_{\alpha}^{2}\left|\nabla w_{\alpha}\right|^{2} d x \leq C$.

Proof. Let $t_{\alpha} \leq T_{\alpha} \leq \operatorname{dist}\left(x_{\alpha}, \partial \Omega\right)$ so that

$$
\sup _{\partial B_{T_{\alpha}}\left(x_{\alpha}\right)} u_{\alpha}^{2}+T_{\alpha} \int_{\partial B_{T_{\alpha}}\left(x_{\alpha}\right)}\left|\nabla u_{\alpha}\right|^{2} d o \leq C
$$

as in the proof of [5], Lemma 5.5.

Compute

$$
\begin{aligned}
-\operatorname{div} & {\left[\left(1+\left|\nabla u_{\alpha}\right|^{2}\right)^{\alpha-1} \nabla\left(\frac{\bar{u}_{\alpha}^{2} w_{\alpha}^{2}}{2}\right)\right]+\left(1+\left|\nabla u_{\alpha}\right|^{2}\right)^{\alpha-1} \bar{u}_{\alpha}^{2}\left|\nabla w_{\alpha}\right|^{2} } \\
= & \bar{u}_{\alpha} w_{\alpha}^{2}\left(-\operatorname{div}\left[\left(1+\left|\nabla u_{\alpha}\right|^{2}\right)^{\alpha-1} \nabla \bar{u}_{\alpha}\right]\right) \\
& +\bar{u}_{\alpha}^{2} w_{\alpha}\left(-\operatorname{div}\left[\left(1+\left|\nabla u_{\alpha}\right|^{2}\right)^{\alpha-1} \nabla w_{\alpha}\right]\right) \\
& -\left(1+\left|\nabla u_{\alpha}\right|^{2}\right)^{\alpha-1}\left|\nabla \bar{u}_{\alpha}\right|^{2} w_{\alpha}^{2}-4\left(1+\left|\nabla u_{\alpha}\right|^{2}\right)^{\alpha-1} \bar{u}_{\alpha} w_{\alpha} \nabla \bar{u}_{\alpha} \nabla w_{\alpha} .
\end{aligned}
$$

The first and second term on the right may be rewritten as

$$
\begin{gathered}
\bar{u}_{\alpha} w_{\alpha}^{2}\left(-\operatorname{div}\left[\left(1+\left|\nabla u_{\alpha}\right|^{2}\right)^{\alpha-1} \nabla \bar{u}_{\alpha}\right]\right) \\
+\bar{u}_{\alpha}^{2} w_{\alpha}\left(-\operatorname{div}\left[\left(1+\left|\nabla u_{\alpha}\right|^{2}\right)^{\alpha-1} \nabla w_{\alpha}\right]\right) \\
=\bar{u}_{\alpha} w_{\alpha} u_{\alpha} f\left(u_{\alpha}\right)-\bar{u}_{\alpha} w_{\alpha}^{2}\left(-\operatorname{div}\left[\left(1+\left|\nabla u_{\alpha}\right|^{2}\right)^{\alpha-1} \nabla w_{\alpha}\right]\right) \\
\quad-\bar{u}_{\alpha}^{2} w_{\alpha}\left(-\operatorname{div}\left[\left(1+\left|\nabla u_{\alpha}\right|^{2}\right)^{\alpha-1} \nabla \bar{u}_{\alpha}\right]\right) \\
=\bar{u}_{\alpha} w_{\alpha} e^{\eta_{\alpha}}+\operatorname{div}\left[\left(1+\left|\nabla u_{\alpha}\right|^{2}\right)^{\alpha-1}\left(\bar{u}_{\alpha} w_{\alpha}^{2} \nabla w_{\alpha}+\bar{u}_{\alpha}^{2} w_{\alpha} \nabla \bar{u}_{\alpha}\right)\right] \\
\quad-\left(1+\left|\nabla u_{\alpha}\right|^{2}\right)^{\alpha-1}\left(\left(\bar{u}_{\alpha}^{2}+w_{\alpha}^{2}\right) \nabla \bar{u}_{\alpha} \nabla w_{\alpha}\right. \\
\left.+2 \bar{u}_{\alpha} w_{\alpha}\left(\left|\nabla w_{\alpha}\right|^{2}+\left|\nabla \bar{u}_{\alpha}\right|^{2}\right)\right) .
\end{gathered}
$$


The last term is the one whose integral is the most difficult to control in the quasilinear case. Noting that

$$
\int_{\partial B_{r}\left(x_{\alpha}\right)} \nabla \bar{u}_{\alpha} \nabla w_{\alpha} d o=0, \int_{\partial B_{r}\left(x_{\alpha}\right)} w_{\alpha} d o=0
$$

for any $r \leq T_{\alpha}$ we have

$$
\begin{aligned}
I:= & \int_{\partial B_{r}\left(x_{\alpha}\right)}\left(1+\left|\nabla u_{\alpha}\right|^{2}\right)^{\alpha-1}\left(\left(\bar{u}_{\alpha}^{2}+w_{\alpha}^{2}\right) \nabla \bar{u}_{\alpha} \nabla w_{\alpha}\right. \\
& \left.+2 \bar{u}_{\alpha} w_{\alpha}\left(\left|\nabla w_{\alpha}\right|^{2}+\left|\nabla \bar{u}_{\alpha}\right|^{2}\right)\right) d o \\
= & \int_{\partial B_{r}\left(x_{\alpha}\right)}\left(\left(1+\left|\nabla u_{\alpha}\right|^{2}\right)^{\alpha-1}-\left(1+\left|\nabla \bar{u}_{\alpha}\right|^{2}\right)^{\alpha-1}\right) \\
& \cdot\left(\left(\bar{u}_{\alpha}^{2}+w_{\alpha}^{2}\right) \nabla \bar{u}_{\alpha} \nabla w_{\alpha}+2 \bar{u}_{\alpha} w_{\alpha}\left(\left|\nabla w_{\alpha}\right|^{2}+\left|\nabla \bar{u}_{\alpha}\right|^{2}\right)\right) d o \\
& +\int_{\partial B_{r}\left(x_{\alpha}\right)}\left(1+\left|\nabla \bar{u}_{\alpha}\right|^{2}\right)^{\alpha-1}\left(w_{\alpha}^{2} \nabla \bar{u}_{\alpha} \nabla w_{\alpha}+2 \bar{u}_{\alpha} w_{\alpha}\left|\nabla w_{\alpha}\right|^{2}\right) d o \\
= & I_{1}+I_{2} .
\end{aligned}
$$

We first deal with the term $I_{2}$.

Observe that Lemmas 4.9 and 4.10 imply that, as $\alpha \searrow 1$,

$$
\left(1+\left|\nabla u_{\alpha}\right|^{2}\right)^{\alpha-1}=\left(1+r_{\alpha}^{-2}\left|\nabla w_{\alpha}^{(1)}\right|^{2}\right)^{\alpha-1} \rightarrow 1
$$

uniformly for $r \leq L r_{\alpha}$, any $L \in \mathbb{N}$. Similarly, by Lemmas 4.10 and 4.14 as $\alpha \rightarrow 1$ we have

$$
\left(1+\left|\nabla u_{\alpha}(y)\right|^{2}\right)^{\alpha-1}=\left(1+r^{-2}\left(r\left|\nabla w_{\alpha}^{(0)}(y)\right|\right)^{2}\right)^{\alpha-1} \rightarrow 1
$$

uniformly for $L r_{\alpha} \leq r \leq T_{\alpha}$ and any $y \in \partial B_{r}\left(x_{\alpha}\right)$, any $L \in \mathbb{N}$. Finally, note that Lemma 4.14 also implies the uniform bound

$$
\left|w_{\alpha}\right| \leq C \text {. }
$$

Thus, and using Young's inequality $2 a b \leq \delta a^{2}+\delta^{-1} b^{2}$ for any $\delta>0$ together with the estimates

$$
\begin{aligned}
& \int_{\partial B_{r}\left(x_{\alpha}\right)}\left|\nabla \bar{u}_{\alpha}\right|^{2} d o \leq \int_{\partial B_{r}\left(x_{\alpha}\right)}\left|\nabla u_{\alpha}\right|^{2} d o, \\
& \int_{\partial B_{r}\left(x_{\alpha}\right)}\left|\nabla w_{\alpha}\right|^{2} d o \leq \int_{\partial B_{r}\left(x_{\alpha}\right)}\left|\nabla u_{\alpha}\right|^{2} d o,
\end{aligned}
$$

for any $r \leq T_{\alpha}$ and any $\delta>0$ with a constant $C=C(\delta)$ we obtain

$$
\begin{aligned}
\left|I_{2}\right| & \leq C \max _{\partial B_{r}\left(x_{\alpha}\right)} w_{\alpha}^{2} \cdot \int_{\partial B_{r}\left(x_{\alpha}\right)}\left|\nabla u_{\alpha}\right|^{2} d o+\delta \int_{\partial B_{r}\left(x_{\alpha}\right)} \bar{u}_{\alpha}^{2}\left|\nabla w_{\alpha}\right|^{2} d o \\
& \leq C \int_{\partial B_{r}\left(x_{\alpha}\right)}\left(1+\left|\nabla u_{\alpha}\right|^{2}\right)^{\alpha} d o+\delta \int_{\partial B_{r}\left(x_{\alpha}\right)} \bar{u}_{\alpha}^{2}\left|\nabla w_{\alpha}\right|^{2} d o .
\end{aligned}
$$


Next we confront the term $I_{1}$.

By the same reasoning as used to bound $I_{2}$, in a preliminary step we may estimate

$$
\begin{aligned}
& \mid I_{1}-\int_{\partial B_{r}\left(x_{\alpha}\right)}\left(\left(1+\left|\nabla u_{\alpha}\right|^{2}\right)^{\alpha-1}-\left(1+\left|\nabla \bar{u}_{\alpha}\right|^{2}\right)^{\alpha-1}\right) \\
& \cdot\left(\bar{u}_{\alpha}^{2} \nabla \bar{u}_{\alpha} \nabla w_{\alpha}+2 \bar{u}_{\alpha} w_{\alpha}\left|\nabla \bar{u}_{\alpha}\right|^{2}\right) d o \mid \\
& \leq C \int_{\partial B_{r}\left(x_{\alpha}\right)}\left(1+\left|\nabla u_{\alpha}\right|^{2}\right)^{\alpha} d o+\delta \int_{\partial B_{r}\left(x_{\alpha}\right)} \bar{u}_{\alpha}^{2}\left|\nabla w_{\alpha}\right|^{2} d o,
\end{aligned}
$$

exhibiting the crucial term. Expand

$$
\begin{aligned}
& \left(1+\left|\nabla u_{\alpha}\right|^{2}\right)^{\alpha-1}-\left(1+\left|\nabla \bar{u}_{\alpha}\right|^{2}\right)^{\alpha-1} \\
& \quad=2(\alpha-1)\left(1+\left|\nabla \bar{u}_{\alpha}+\vartheta \nabla w_{\alpha}\right|^{2}\right)^{\alpha-2}\left(\nabla \bar{u}_{\alpha}+\vartheta \nabla w_{\alpha}\right) \nabla w_{\alpha}
\end{aligned}
$$

with some function $0 \leq \vartheta \leq 1$ and write

$$
\nabla \bar{u}_{\alpha}=\left(\nabla \bar{u}_{\alpha}+\vartheta \nabla w_{\alpha}\right)-\vartheta \nabla w_{\alpha}
$$

to obtain

$$
\begin{aligned}
I_{11}:=\mid & \int_{\partial B_{r}\left(x_{\alpha}\right)}\left(\left(1+\left|\nabla u_{\alpha}\right|^{2}\right)^{\alpha-1}-\left(1+\left|\nabla \bar{u}_{\alpha}\right|^{2}\right)^{\alpha-1}\right) \bar{u}_{\alpha}^{2} \nabla \bar{u}_{\alpha} \nabla w_{\alpha} d o \mid \\
\leq & 2(\alpha-1) \int_{\partial B_{r}\left(x_{\alpha}\right)}\left(1+\left|\nabla \bar{u}_{\alpha}+\vartheta \nabla w_{\alpha}\right|^{2}\right)^{\alpha-1} \bar{u}_{\alpha}^{2}\left|\nabla w_{\alpha}\right|^{2} d o \\
& +\int_{\partial B_{r}\left(x_{\alpha}\right)}\left|\left(1+\left|\nabla u_{\alpha}\right|^{2}\right)^{\alpha-1}-\left(1+\left|\nabla \bar{u}_{\alpha}\right|^{2}\right)^{\alpha-1}\right| \bar{u}_{\alpha}^{2}\left|\nabla w_{\alpha}\right|^{2} d o .
\end{aligned}
$$

Moreover, estimating

$$
\left|\nabla \bar{u}_{\alpha}\right|^{2} \leq\left|\nabla \bar{u}_{\alpha}\right|\left(\left|\nabla \bar{u}_{\alpha}+\vartheta \nabla w_{\alpha}\right|+\vartheta\left|\nabla w_{\alpha}\right|\right),
$$

we find

$$
\begin{aligned}
I_{12} & :=\left.\left|\int_{\partial B_{r}\left(x_{\alpha}\right)}\left(\left(1+\left|\nabla u_{\alpha}\right|^{2}\right)^{\alpha-1}-\left(1+\left|\nabla \bar{u}_{\alpha}\right|^{2}\right)^{\alpha-1}\right) \bar{u}_{\alpha} w_{\alpha}\right| \nabla \bar{u}_{\alpha}\right|^{2} d o \mid \\
& \leq 2(\alpha-1) \int_{\partial B_{r}\left(x_{\alpha}\right)}\left(1+\left|\nabla \bar{u}_{\alpha}+\vartheta \nabla w_{\alpha}\right|^{2}\right)^{\alpha-1} \bar{u}_{\alpha}\left|w_{\alpha}\right|\left|\nabla \bar{u}_{\alpha}\right|\left|\nabla w_{\alpha}\right| d o \\
& +\int_{\partial B_{r}\left(x_{\alpha}\right)}\left|\left(1+\left|\nabla u_{\alpha}\right|^{2}\right)^{\alpha-1}-\left(1+|\nabla \bar{u}|^{2}\right)^{\alpha-1}\right| \bar{u}_{\alpha}\left|w_{\alpha}\right|\left|\nabla \bar{u}_{\alpha}\right|\left|\nabla w_{\alpha}\right| d o .
\end{aligned}
$$


Thus, and recalling (80), (81), and (82), we obtain that

$$
\begin{aligned}
\left|I_{1}\right| \leq I_{11}+I_{12} & \leq o(1) \int_{\partial B_{r}\left(x_{\alpha}\right)}\left(\bar{u}_{\alpha}^{2}\left|\nabla w_{\alpha}\right|^{2}+\left|w_{\alpha}\right|^{2}\left|\nabla \bar{u}_{\alpha}\right|^{2}\right) d o \\
& \leq o(1) \int_{\partial B_{r}\left(x_{\alpha}\right)} \bar{u}_{\alpha}^{2}\left|\nabla w_{\alpha}\right|^{2} d o+C \int_{\partial B_{r}\left(x_{\alpha}\right)}\left(1+\left|\nabla u_{\alpha}\right|^{2}\right)^{\alpha} d o,
\end{aligned}
$$

where $o(1) \rightarrow 0$ uniformly for $r \leq T_{\alpha}$ as $\alpha \rightarrow 1$.

Summarizing, for any $\delta>0$ and sufficiently small $\alpha>1$ we therefore obtain that

$$
|I| \leq C \int_{\partial B_{r}\left(x_{\alpha}\right)}\left(1+\left|\nabla u_{\alpha}\right|^{2}\right)^{\alpha} d o+\delta \int_{\partial B_{r}\left(x_{\alpha}\right)} \bar{u}_{\alpha}^{2}\left|\nabla w_{\alpha}\right|^{2} d o .
$$

As in the proof of Lemma 5.5 in [5], and using Lemma 4.13 in the final estimate, for $L r_{\alpha} \leq r \leq T_{\alpha}$ we may bound

$$
\begin{aligned}
I I: & =\int_{\partial B_{r}\left(x_{\alpha}\right)} \bar{u}_{\alpha} w_{\alpha} e^{\eta_{\alpha}} d o=\int_{\partial B_{r}\left(x_{\alpha}\right)} \bar{u}_{\alpha} w_{\alpha}\left(e^{\eta_{\alpha}}-e^{\bar{\eta}_{\alpha}}\right) d o \\
& \leq \max _{\partial B_{r}\left(x_{\alpha}\right)}\left(r^{2} e^{\eta_{\alpha}}\right) r^{-2} \int_{\partial B_{r}\left(x_{\alpha}\right)} \bar{u}_{\alpha}^{2} w_{\alpha}^{2} d o \\
& \leq \delta \int_{\partial B_{r}\left(x_{\alpha}\right)} \bar{u}_{\alpha}^{2}\left|\nabla w_{\alpha}\right|^{2} d o+C(\delta) \int_{\partial B_{r}\left(x_{\alpha}\right)}\left|\nabla u_{\alpha}\right|^{2} d o
\end{aligned}
$$

and

$$
I I \leq C \int_{\partial B_{r}\left(x_{\alpha}\right)} \bar{u}_{\alpha}^{2}\left|\nabla w_{\alpha}\right|^{2} d o
$$

for $r \leq L r_{\alpha}$.

Also splitting the last term in (78)

$$
4 \bar{u}_{\alpha} w_{\alpha} \nabla \bar{u}_{\alpha} \nabla w_{\alpha} \leq C(\delta) w_{\alpha}^{2}\left|\nabla \bar{u}_{\alpha}\right|^{2}+\delta \bar{u}_{\alpha}^{2}\left|\nabla w_{\alpha}\right|^{2},
$$

and choosing $\delta=\frac{1}{6}$, from (76)-(81) for any $L \in \mathbb{N}$ we then obtain

$$
\begin{aligned}
& \frac{1}{2} \int_{B_{T_{\alpha}\left(x_{\alpha}\right)}}\left(1+\left|\nabla u_{\alpha}\right|^{2}\right)^{\alpha-1} \bar{u}_{\alpha}^{2}\left|\nabla w_{\alpha}\right|^{2} d x \\
& \leq \int_{\partial B_{T_{\alpha}}\left(x_{\alpha}\right)}\left(1+\left|\nabla u_{\alpha}\right|^{2}\right)^{\alpha-1}\left(\partial_{n}\left(\frac{\bar{u}_{\alpha}^{2} w_{\alpha}^{2}}{2}\right)+\bar{u}_{\alpha} w_{\alpha}^{2} \partial_{n} w_{\alpha}+\bar{u}_{\alpha}^{2} w_{\alpha} \partial_{n} \bar{u}_{\alpha}\right) d o \\
& \quad+C \int_{B_{T_{\alpha}\left(x_{\alpha}\right)}}\left(1+\left|\nabla u_{\alpha}\right|^{2}\right)^{\alpha} d x+C \int_{B_{L r_{\alpha}\left(x_{\alpha}\right)}} \bar{u}_{\alpha}^{2}\left|\nabla w_{\alpha}\right|^{2} d x \\
& \leq C+C \int_{B_{L r_{\alpha}\left(x_{\alpha}\right)}}\left|\nabla\left(\eta_{\alpha}-\bar{\eta}_{\alpha}\right)\right|^{2} d x \\
& =C+C \int_{B_{L}(0)}\left|\nabla\left(\eta_{\alpha}^{(1)}-\bar{\eta}_{\alpha}^{(1)}\right)\right|^{2} d x=C+o(1),
\end{aligned}
$$

with error $o(1) \rightarrow 0$ as $\alpha \rightarrow 1$, proving the claim. 
A final auxiliary argument is needed before we can complete the proof.

Lemma 4.16. For $0 \leq r \leq t_{\alpha}$, with error o(1) $\rightarrow 0$ uniformly in $r$ as $\alpha \rightarrow 1$ there holds

$$
\begin{aligned}
-\int_{\partial B_{r}\left(x_{\alpha}\right)} \partial_{n} \eta_{\alpha} d o= & 8 \pi \int_{B_{r}\left(x_{\alpha}\right)}\left(e^{\eta_{\alpha}}-\left|\nabla u_{\alpha}\right|^{2}\right) d x \\
& +o(1)\left(r \int_{\partial B_{r}\left(x_{\alpha}\right)}\left|\nabla\left(\eta_{\alpha}-\bar{\eta}_{\alpha}\right)\right|^{2} d o\right)^{1 / 2}+o(1),
\end{aligned}
$$

where $\partial_{n} \eta_{\alpha}$ is the outward normal derivative.

Proof. Compute

$$
\begin{aligned}
\operatorname{div}((1 & \left.\left.+\left|\nabla u_{\alpha}\right|^{2}\right)^{\alpha-1} \nabla \eta_{\alpha}\right)=\left(\varphi^{\prime}\left(u_{\alpha}\right)+\frac{2}{u_{\alpha}}\right) \operatorname{div}\left(\left(1+\left|\nabla u_{\alpha}\right|^{2}\right)^{\alpha-1} \nabla u_{\alpha}\right) \\
& +\left(\varphi^{\prime \prime}\left(u_{\alpha}\right)-\frac{2}{u_{\alpha}^{2}}\right)\left(1+\left|\nabla u_{\alpha}\right|^{2}\right)^{\alpha-1}\left|\nabla u_{\alpha}\right|^{2} \\
= & -\left(\frac{\varphi^{\prime}\left(u_{\alpha}\right)}{u_{\alpha}}+\frac{2}{u_{\alpha}^{2}}\right) u_{\alpha} f\left(u_{\alpha}\right) \\
& +\left(\varphi^{\prime \prime}\left(u_{\alpha}\right)-\frac{2}{u_{\alpha}^{2}}\right)\left(1+\left|\nabla u_{\alpha}\right|^{2}\right)^{\alpha-1}\left|\nabla u_{\alpha}\right|^{2} .
\end{aligned}
$$

By Lemma 4.12, as $\alpha \rightarrow 1$ we have $\inf _{B_{t_{\alpha}}\left(x_{\alpha}\right)} u_{\alpha} \rightarrow \infty$. Hence the properties of $\varphi$ and our uniform estimates (80), (81) imply that

$\frac{\varphi^{\prime}\left(u_{\alpha}\right)}{u_{\alpha}}+\frac{2}{u_{\alpha}^{2}}=8 \pi+o(1),\left(\varphi^{\prime \prime}\left(u_{\alpha}\right)-\frac{2}{u_{\alpha}^{2}}\right)\left(1+\left|\nabla u_{\alpha}\right|^{2}\right)^{\alpha-1}=8 \pi+o(1)$ with error $o(1) \rightarrow 0$ uniformly on $B_{t_{\alpha}}\left(x_{\alpha}\right)$ as $\alpha \rightarrow 1$. Upon integration it follows that

$-\int_{\partial B_{r}\left(x_{\alpha}\right)}\left(1+\left|\nabla u_{\alpha}\right|^{2}\right)^{\alpha-1} \partial_{n} \eta_{\alpha} d o=8 \pi \int_{B_{r}\left(x_{\alpha}\right)}\left(e^{\eta_{\alpha}}-\left|\nabla u_{\alpha}\right|^{2}\right) d x+o(1)$ with error $o(1) \rightarrow 0$ uniformly for $r \leq t_{\alpha}$ as $\alpha \rightarrow 1$.

Estimating

$$
\left|\partial_{n} \eta_{\alpha}\right| \leq\left|\partial_{n}\left(\eta_{\alpha}-\bar{\eta}_{\alpha}\right)\right|+\left|\partial_{n} \bar{\eta}_{\alpha}\right|
$$

and recalling that $\left(1+\left|\nabla u_{\alpha}\right|^{2}\right)^{\alpha-1} \rightarrow 1$ uniformly on $B_{t_{\alpha}}\left(x_{\alpha}\right)$, we find that with error $o(1) \rightarrow 0$ as $\alpha \rightarrow 1$

$$
\begin{aligned}
\int_{\partial B_{r}\left(x_{\alpha}\right)}[(1 & \left.\left.+\left|\nabla u_{\alpha}\right|^{2}\right)^{\alpha-1}-1\right] \partial_{n} \eta_{\alpha} d o \\
& \leq o(1)\left(\int_{\partial B_{r}\left(x_{\alpha}\right)}\left|\partial_{n} \eta_{\alpha}-\partial_{n} \bar{\eta}_{\alpha}\right| d o+\int_{\partial B_{r}\left(x_{\alpha}\right)}\left|\partial_{n} \bar{\eta}_{\alpha}\right| d o\right) \\
& \leq o(1)\left(r \int_{\partial B_{r}\left(x_{\alpha}\right)}\left|\nabla\left(\eta_{\alpha}-\bar{\eta}_{\alpha}\right)\right|^{2} d o\right)^{1 / 2}+o(1) \int_{\partial B_{r}\left(x_{\alpha}\right)} \partial_{n} \eta_{\alpha} d o
\end{aligned}
$$


uniformly for $r \leq t_{\alpha}$. Hence we obtain

$$
\begin{aligned}
-\int_{\partial B_{r}\left(x_{\alpha}\right)} \partial_{n} \eta_{\alpha} d o & =8 \pi \int_{B_{r}\left(x_{\alpha}\right)}\left(e^{\eta_{\alpha}}-\left|\nabla u_{\alpha}\right|^{2}\right) d x \\
& +o(1)\left(r \int_{\partial B_{r}\left(x_{\alpha}\right)}\left|\nabla\left(\eta_{\alpha}-\bar{\eta}_{\alpha}\right)\right|^{2} d o\right)^{1 / 2} \\
& +o(1) \int_{\partial B_{r}\left(x_{\alpha}\right)} \partial_{n} \eta_{\alpha} d o+o(1)
\end{aligned}
$$

where $o(1) \rightarrow 0$ as $\alpha \rightarrow 1$, uniformly in $r$. The claim follows.

Lemma 4.17. There holds $\beta=\frac{1}{2}$.

Proof. For $\eta_{\alpha}=\eta_{\alpha}^{(0)}$ as above, let

$$
\begin{aligned}
& \Phi_{\alpha}(r)=\int_{B_{r}\left(x_{\alpha}\right)} e^{\eta_{\alpha}} d x=\int_{B_{1}(0)} e^{\eta_{\alpha}\left(x_{\alpha}+r \xi\right)} r^{2} d \xi, \\
& \Psi_{\alpha}(r)=\int_{B_{r}\left(x_{\alpha}\right)}\left|\nabla u_{\alpha}\right|^{2} d x .
\end{aligned}
$$

Shifting $x_{\alpha}$ to the origin for convenience, then we have

$$
r \Phi_{\alpha}^{\prime}(r)=2 \Phi_{\alpha}(r)+\int_{0}^{r}\left(\int_{\partial B_{\rho}(0)} e^{\eta_{\alpha}} \rho \partial_{n} \eta_{\alpha} d o\right) d \rho,
$$

where $\partial_{n} \eta_{\alpha}$ is the outward normal derivative on $\partial B_{\rho}(0)$. Now for $0 \leq \rho \leq r$ we may write

$$
\begin{aligned}
\int_{\partial B_{\rho}(0)} e^{\eta_{\alpha}} \rho \partial_{n} \eta_{\alpha} d o= & \int_{\partial B_{\rho}(0)} e^{\eta_{\alpha}} \rho \partial_{n}\left(\eta_{\alpha}-\bar{\eta}_{\alpha}\right) d o+\int_{\partial B_{\rho}(0)} e^{\eta_{\alpha}} \rho \partial_{n} \bar{\eta}_{\alpha} d o \\
= & \int_{\partial B_{\rho}(0)}\left(e^{\eta_{\alpha}}-e^{\bar{\eta}_{\alpha}}\right) \rho \partial_{n}\left(\eta_{\alpha}-\bar{\eta}_{\alpha}\right) d o \\
& +\int_{\partial B_{\rho}(0)} e^{\eta_{\alpha}} d o \cdot \frac{1}{2 \pi} \int_{\partial B_{\rho}(0)} \partial_{n} \eta_{\alpha} d o .
\end{aligned}
$$

By (75), Lemmas 4.13 and 4.15, and the Poincaré inequality

$$
\begin{aligned}
\int_{\partial B_{\rho}(0)} & \left(e^{\eta_{\alpha}}-e^{\bar{\eta}_{\alpha}}\right) \rho \partial_{n}\left(\eta_{\alpha}-\bar{\eta}_{\alpha}\right) d o \\
& \leq \max _{\partial B_{\rho}(0)}\left(\rho^{2} e^{\eta_{\alpha}}\right) \cdot \int_{\partial B_{\rho}(0)} \frac{\left|\eta_{\alpha}-\bar{\eta}_{\alpha}\right|}{\rho}\left|\nabla\left(\eta_{\alpha}-\bar{\eta}_{\alpha}\right)\right| d o \\
& \leq o(1) \int_{\partial B_{\rho}(0)}\left(\frac{\left|\eta_{\alpha}-\bar{\eta}_{\alpha}\right|^{2}}{\rho^{2}}+\left|\nabla\left(\eta_{\alpha}-\bar{\eta}_{\alpha}\right)\right|^{2}\right) d o \\
& \leq o(1) \int_{\partial B_{\rho}(0)}\left|\nabla\left(\eta_{\alpha}-\bar{\eta}_{\alpha}\right)\right|^{2} d o,
\end{aligned}
$$


with $o(1) \rightarrow 0$ uniformly for $L r_{\alpha} \leq r \leq t_{\alpha}$ and $1<\alpha \leq \alpha(L) \rightarrow 1$ as $L \rightarrow \infty$. Hence the integral of this term from 0 to any $r \leq t_{\alpha}$ vanishes in the limit $\alpha \rightarrow 1$.

Moreover, from Lemma 4.16 we obtain

$$
\begin{aligned}
\int_{\partial B_{\rho}(0)} \partial_{n} \eta_{\alpha} d o= & 8 \pi\left(\Psi_{\alpha}(\rho)-\Phi_{\alpha}(\rho)\right) \\
& +o(1)\left(\rho \int_{\partial B_{\rho}(0)}\left|\nabla\left(\eta_{\alpha}-\bar{\eta}_{\alpha}\right)\right|^{2} d o\right)^{1 / 2}+o(1),
\end{aligned}
$$

where $o(1) \rightarrow 0$ as $\alpha \rightarrow 1$, uniformly for $\rho \leq t_{\alpha}$. By Hölder's inequality, (75), and Lemma 4.15 for any $r \leq t_{\alpha}$ we can estimate

$$
\begin{aligned}
& \left(\int_{0}^{r} \int_{\partial B_{\rho}(0)} e^{\eta_{\alpha}} d o\left(\rho \int_{\partial B_{\rho}(0)}\left|\nabla\left(\eta_{\alpha}-\bar{\eta}_{\alpha}\right)\right|^{2} d o\right)^{1 / 2} d \rho\right)^{2} \\
& \quad \leq \int_{0}^{r} \rho\left(\int_{\partial B_{\rho}(0)} e^{\eta_{\alpha}} d o\right)^{2} d \rho \cdot \int_{B_{r}(0)}\left|\nabla\left(\eta_{\alpha}-\bar{\eta}_{\alpha}\right)\right|^{2} d x \\
& \quad \leq C \int_{0}^{r} \rho^{2} \int_{\partial B_{\rho}(0)} e^{2 \eta_{\alpha}} d o d \rho \leq C \max _{B_{t_{\alpha}}\left(x_{\alpha}\right)}\left(|y|^{2} e^{\eta_{\alpha}(y)}\right) \int_{B_{\rho}(0)} e^{\eta_{\alpha}} d x \\
& \quad \leq C \Phi_{\alpha}(\rho) \leq C .
\end{aligned}
$$

Hence we find

$$
\begin{aligned}
0 & \leq r \Phi_{\alpha}^{\prime}(r)=2 \Phi_{\alpha}(r)-4 \int_{0}^{r} \Phi_{\alpha}^{\prime}(\rho)\left(\Phi_{\alpha}(\rho)-\Psi_{\alpha}(\rho)\right) d \rho+o(1) \\
& =2 \Phi_{\alpha}(r)\left(1-\Phi_{\alpha}(r)\right)+4 \int_{0}^{r} \Psi_{\alpha}^{\prime}(\rho)\left(\Phi_{\alpha}(r)-\Phi_{\alpha}(\rho)\right) d \rho+o(1)
\end{aligned}
$$

where $o(1) \rightarrow 0$ as $\alpha \rightarrow 1$.

Splitting the last integral

$$
\int_{0}^{r} \Psi_{\alpha}^{\prime}(\rho)\left(\Phi_{\alpha}(r)-\Phi_{\alpha}(\rho)\right) d \rho=\int_{0}^{L r_{\alpha}} \ldots+\int_{L r_{\alpha}}^{r} \ldots
$$

and taking account of Lemma 4.9, (72), and (73), as in [5] we may estimate

$$
\begin{aligned}
& \int_{0}^{r} \Psi_{\alpha}^{\prime}(\rho)\left(\Phi_{\alpha}(r)-\Phi_{\alpha}(\rho)\right) d \rho \\
& \quad \leq \int_{0}^{r} \Psi_{\alpha}^{\prime}(\rho)\left(\Phi_{\alpha}(r)-1\right) d \rho+o(1)=\Psi_{\alpha}(r)\left(\Phi_{\alpha}(r)-1\right)+o(1)
\end{aligned}
$$

to obtain the inequality

$$
0 \leq r \Phi_{\alpha}^{\prime}(r) \leq 2\left(\Phi_{\alpha}(r)-2 \Psi_{\alpha}(r)\right)\left(1-\Phi_{\alpha}(r)\right)+o(1)
$$


with error $o(1) \rightarrow 0$ as $\alpha \rightarrow 1$. Evaluating at $r=t_{\alpha}$, from Lemma 4.12 we have

$$
\Phi_{\alpha}\left(t_{\alpha}\right)-2 \Psi_{\alpha}\left(t_{\alpha}\right) \geq 2 \beta-2(2 \beta-1)+o(1)=2(1-\beta)+o(1)>0
$$

for sufficiently small $\alpha>1$. We conclude that $\Phi_{\alpha}\left(t_{\alpha}\right) \leq 1+o(1)$ and hence $\beta=1 / 2$, as desired.

\section{References}

1. Adimurthi: Existence of positive solutions of the semilinear Dirichlet problem with critical growth for the $n$-laplacian. Ann. Sc. Norm. Sup. Pisa, Ser. IV, 17, 393-413 (1990)

2. Adimurthi, S. Prashanth: Failure of Palais-Smale condition and blow-up analysis for the critical exponent problem in $\mathbb{R}^{2}$ (preprint)

3. Adimurthi, S. Prashanth: Critical exponent problem in $\mathbb{R}^{2}$, border-line between existence and non-existence of positive solutions for Dirichlet problem. Advances Diff. Eqs. (to appear)

4. Adimurthi, P.N. Srikanth, S.L. Yadava: Phenomena of critical exponent in $\mathbb{R}^{2}$. Proc. Roy. Soc. Edinburgh, 199 A, 19-25 (1991)

5. Adimurthi, M. Struwe: Global compactness properties of semilinear elliptic equations with critical exponential growth. J. Funct. Analysis (in press)

6. A. Bahri, J.-M. Coron: On a nonlinear elliptic equation involving the critical Sobolev exponent: The effect of topology of the domain. Comm. Pure Appl. Math. 41, 253-294 (1988)

7. H. Brezis, F. Merle: Uniform estimates and blow-up behavior for solutions of $-\Delta u=$ $V(x) e^{u}$ in two dimensions. Comm. Partial Differential Equations 16, 1223-1253 (1991)

8. L.A. Caffarelli, X. Cabré: Fully nonlinear elliptic equations. AMS Coll. Publ. 43 (1995)

9. S.Y.A. Chang, P. Yang: Conformal deformation of metric on $S^{2}$. J.D.G. 27, 259-296 (1987)

10. W. Chen, C. Li: Classification of solutions of some nonlinear elliptic equations. Duke Math. J. 63, 615-623 (1991)

11. J.-M. Coron: Topologie et cas limité des injections de Sobolev. C.R. Acad. Sc. Paris 299, Ser. I, 209-212 (1984)

12. D.G. de Figueiredo, B. Ruf: Existence and non-existence of radial solutions for elliptic equations with critical exponent in $\mathbb{R}^{2}$. Comm. Pure Appl. Math. 48, 639-655 (1995)

13. I. Ekeland: On the variational principle. J. Math. Anal. Appl. 47, 324-353 (1974)

14. A. Fiorenza, C. Sbordone: Existence and uniqueness results for solutions of nonlinear equations with right hand side in $L^{1}$. Studia Math. 127, 223-231 (1998)

15. B. Kawohl: Rearrangements and convexity of level sets in partial differential equation. Lect. Notes Math. 1150 (1985), Springer

16. R.S. Palais: Lusternik-Schnirelman theory on Banach manifolds. Topology 5, 115-132 (1966)

17. Y. Li, I. Shafrir: Blow-up analysis for solutions of $-\Delta u=V e^{u}$ in dimension two. Indiana Univ. Math. J. 43(4), 1255-1270 (1994)

18. J. Moser: A sharp form of an inequality by N. Trudinger. Indiana Univ. Math. J. 20, 1077-1092 (1971)

19. J. Sacks, K. Uhlenbeck: The existence of minimal immersions of 2-spheres. Ann. Math. 113, 1-24 (1981)

20. M. Struwe: A global compactness result for elliptic boundary value problems involving limiting nonlinearities. Math. Z. 187, 511-517 (1984) 
21. M. Struwe: Critical points of embeddings of $H_{0}^{1, n}$ into Orlicz spaces. Ann. Inst. H. Poincaré, Analyse Non Linéaire 5, 425-464 (1988)

22. M. Struwe: The existence of surfaces of constant mean curvature with free boundaries. Acta Math. 160, 19-64 (1988)

23. M. Struwe: Variational methods. Springer (1990); 2nd edition, Ergebnisse Math. Grenzgebiete 34 (1996)

24. N.S. Trudinger: On embeddings into Orlicz spaces and some applications. J. Math. Mech. 17, 473-484 (1967) 